NBER WORKING PAPER SERIES

\title{
EMPIRICAL ASSET PRICING VIA MACHINE LEARNING
}

\author{
Shihao Gu \\ Bryan Kelly \\ Dacheng Xiu \\ Working Paper 25398 \\ http://www.nber.org/papers/w25398

\section{NATIONAL BUREAU OF ECONOMIC RESEARCH \\ 1050 Massachusetts Avenue} \\ Cambridge, MA 02134 \\ December 2018, Revised September 2019
}

We benefitted from discussions with Joseph Babcock, Si Chen (Discussant), Rob Engle, Andrea Frazzini, Amit Goyal (Discussant), Lasse Pedersen, Lin Peng (Discussant), Alberto Rossi (Discussant), Guofu Zhou (Discussant), and seminar and conference participants at Erasmus School of Economics, NYU, Northwestern, Imperial College, National University of Singapore, UIBE, Nanjing University, Tsinghua PBC School of Finance, Fannie Mae, U.S. Securities and Exchange Commission, City University of Hong Kong, Shenzhen Finance Institute at CUHK, NBER Summer Institute, New Methods for the Cross Section of Returns Conference, Chicago Quantitative Alliance Conference, Norwegian Financial Research Conference, EFA, China International Conference in Finance, 10th World Congress of the Bachelier Finance Society, Financial Engineering and Risk Management International Symposium, Toulouse Financial Econometrics Conference, Chicago Conference on New Aspects of Statistics, Financial Econometrics, and Data Science, Tsinghua Workshop on Big Data and Internet Economics, Q group, IQ-KAP Research Prize Symposium, Wolfe Re- search, INQUIRE UK, Australasian Finance and Banking Conference, Goldman Sachs Global Alternative Risk Premia Conference, AFA, and Swiss Finance Institute. We gratefully acknowledge the computing support from the Research Computing Center at the University of Chicago. The views expressed herein are those of the authors and do not necessarily reflect the views of the National Bureau of Economic Research.

At least one co-author has disclosed a financial relationship of potential relevance for this research. Further information is available online at http://www.nber.org/papers/w25398.ack

NBER working papers are circulated for discussion and comment purposes. They have not been peer-reviewed or been subject to the review by the NBER Board of Directors that accompanies official NBER publications.

(C) 2018 by Shihao Gu, Bryan Kelly, and Dacheng Xiu. All rights reserved. Short sections of text, not to exceed two paragraphs, may be quoted without explicit permission provided that full credit, including $(\mathcal{C}$ notice, is given to the source. 
Empirical Asset Pricing via Machine Learning

Shihao Gu, Bryan Kelly, and Dacheng Xiu

NBER Working Paper No. 25398

December 2018, Revised September 2019

JEL No. C45,C55,C58,G11,G12

\begin{abstract}
We perform a comparative analysis of machine learning methods for the canonical problem of empirical asset pricing: measuring asset risk premia. We demonstrate large economic gains to investors using machine learning forecasts, in some cases doubling the performance of leading regression-based strategies from the literature. We identify the best performing methods (trees and neural networks) and trace their predictive gains to allowance of nonlinear predictor interactions that are missed by other methods. All methods agree on the same set of dominant predictive signals which includes variations on momentum, liquidity, and volatility. Improved risk premium measurement through machine learning simplifies the investigation into economic mechanisms of asset pricing and highlights the value of machine learning in financial innovation.

Shihao Gu

University of Chicago Booth School of Business

5807 S. Woodlawn

Chicago, IL 60637

shihaogu@chicagobooth.edu

Bryan Kelly

Yale School of Management

165 Whitney Ave.

New Haven, CT 06511

and NBER

bryan.kelly@yale.edu

Dacheng Xiu

Booth School of Business

University of Chicago

5807 South Woodlaswn Avenue

Chicago, IL 60637

dachxiu@chicagobooth.edu
\end{abstract}




\section{Introduction}

In this article, we conduct a comparative analysis of machine learning methods for finance. We do so in the context of perhaps the most widely studied problem in finance, that of measuring equity risk premia.

\subsection{Primary Contributions}

Our primary contributions are two-fold. First, we provide a new set of benchmarks for the predictive accuracy of machine learning methods in measuring risk premia of the aggregate market and individual stocks. This accuracy is summarized two ways. The first is a high out-of-sample predictive $R^{2}$ relative to preceding literature that is robust across a variety of machine learning specifications. Second, and more importantly, we demonstrate the large economic gains to investors using machine learning forecasts. A portfolio strategy that times the S\&P 500 with neural network forecasts enjoys an annualized out-of-sample Sharpe ratio of 0.77, versus the 0.51 Sharpe ratio of a buy-and-hold investor. And a value-weighted long-short decile spread strategy that takes positions based on stocklevel neural network forecasts earns an annualized out-of-sample Sharpe ratio of 1.35, more than doubling the performance of a leading regression-based strategy from the literature.

Return prediction is economically meaningful. The fundamental goal of asset pricing is to understand the behavior of risk premia. ${ }^{1}$ If expected returns were perfectly observed, we would still need theories to explain their behavior and empirical analysis to test those theories. But risk premia are notoriously difficult to measure - market efficiency forces return variation to be dominated by unforecastable news that obscures risk premia. Our research highlights gains that can be achieved in prediction and identifies the most informative predictor variables. This helps resolve the problem of risk premium measurement, which then facilitates more reliable investigation into economic mechanisms of asset pricing.

Second, we synthesize the empirical asset pricing literature with the field of machine learning. Relative to traditional empirical methods in asset pricing, machine learning accommodates a far more expansive list of potential predictor variables and richer specifications of functional form. It is this flexibility that allows us to push the frontier of risk premium measurement. Interest in machine learning methods for finance has grown tremendously in both academia and industry. This article provides a comparative overview of machine learning methods applied to the two canonical problems of empirical asset pricing: predicting returns in the cross section and time series. Our view is that the best way for researchers to understand the usefulness of machine learning in the field of asset pricing is to apply and compare the performance of each of its methods in familiar empirical problems.

\footnotetext{
${ }^{1}$ Our focus is on measuring conditional expected stock returns in excess of the risk-free rate. Academic finance traditionally refers to this quantity as the "risk premium" due to its close connection with equilibrium compensation for bearing equity investment risk. We use the terms "expected return" and "risk premium" interchangeably. One may be interested in potentially distinguishing among different components of expected returns such as those due to systematic risk compensation, idiosyncratic risk compensation, or even due to mispricing. For machine learning approaches to this problem, see Gu et al. (2019) and Kelly et al. (2019).
} 


\subsection{What is Machine Learning?}

The definition of "machine learning" is inchoate and is often context specific. We use the term to describe (i) a diverse collection of high-dimensional models for statistical prediction, combined with (ii) so-called "regularization" methods for model selection and mitigation of overfit, and (iii) efficient algorithms for searching among a vast number of potential model specifications.

The high-dimensional nature of machine learning methods (element (i) of this definition) enhances their flexibility relative to more traditional econometric prediction techniques. This flexibility brings hope of better approximating the unknown and likely complex data generating process underlying equity risk premia. With enhanced flexibility, however, comes a higher propensity of overfitting the data. Element (ii) of our machine learning definition describes refinements in implementation that emphasize stable out-of-sample performance to explicitly guard against overfit. Finally, with many predictors it becomes infeasible to exhaustively traverse and compare all model permutations. Element (iii) describes clever machine learning tools designed to approximate an optimal specification with manageable computational cost.

\subsection{Why Apply Machine Learning to Asset Pricing?}

A number of aspects of empirical asset pricing make it a particularly attractive field for analysis with machine learning methods.

1) Two main research agendas have monopolized modern empirical asset pricing research. The first seeks to describe and understand differences in expected returns across assets. The second focuses on dynamics of the aggregate market equity risk premium. Measurement of an asset's risk premium is fundamentally a problem of prediction - the risk premium is the conditional expectation of a future realized excess return. Machine learning, whose methods are largely specialized for prediction tasks, is thus ideally suited to the problem of risk premium measurement.

2) The collection of candidate conditioning variables for the risk premium is large. The profession has accumulated a staggering list of predictors that various researchers have argued possess forecasting power for returns. The number of stock-level predictive characteristics reported in the literature numbers in the hundreds and macroeconomic predictors of the aggregate market number in the dozens. $^{2}$ Additionally, predictors are often close cousins and highly correlated. Traditional prediction methods break down when the predictor count approaches the observation count or predictors are highly correlated. With an emphasis on variable selection and dimension reduction techniques, machine learning is well suited for such challenging prediction problems by reducing degrees of freedom and condensing redundant variation among predictors.

3) Further complicating the problem is ambiguity regarding functional forms through which the high-dimensional predictor set enter into risk premia. Should they enter linearly? If nonlinearities

\footnotetext{
${ }^{2}$ Green et al. (2013) count 330 stock-level predictive signals in published or circulated drafts. Harvey et al. (2016) study 316 "factors," which include firm characteristics and common factors, for describing stock return behavior. They note that this is only a subset of those studied in the literature. Welch and Goyal (2008) analyze nearly 20 predictors for the aggregate market return. In both stock and aggregate return predictions, there presumably exists a much larger set of predictors that were tested but failed to predict returns and were thus never reported.
} 
are needed, which form should they take? Must we consider interactions among predictors? Such questions rapidly proliferate the set of potential model specifications. The theoretical literature offers little guidance for winnowing the list of conditioning variables and functional forms. Three aspects of machine learning make it well suited for problems of ambiguous functional form. The first is its diversity. As a suite of dissimilar methods it casts a wide net in its specification search. Second, with methods ranging from generalized linear models to regression trees and neural networks, machine learning is explicitly designed to approximate complex nonlinear associations. Third, parameter penalization and conservative model selection criteria complement the breadth of functional forms spanned by these methods in order to avoid overfit biases and false discovery.

\subsection{What Specific Machine Learning Methods Do We Study?}

We select a set of candidate models that are potentially well suited to address the three empirical challenges outlined above. They constitute the canon of methods one would encounter in a graduate level machine learning textbook. ${ }^{3}$ This includes linear regression, generalized linear models with penalization, dimension reduction via principal components regression (PCR) and partial least squares (PLS), regression trees (including boosted trees and random forests), and neural networks. This is not an exhaustive analysis of all methods. For example, we exclude support vector machines as these share an equivalence with other methods that we study ${ }^{4}$ and are primarily used for classification problems. Nonetheless, our list is designed to be representative of predictive analytics tools from various branches of the machine learning toolkit.

\subsection{Main Empirical Findings}

We conduct a large scale empirical analysis, investigating nearly 30,000 individual stocks over 60 years from 1957 to 2016. Our predictor set includes 94 characteristics for each stock, interactions of each characteristic with eight aggregate time series variables, and 74 industry sector dummy variables, totaling more than 900 baseline signals. Some of our methods expand this predictor set much further by including nonlinear transformations and interactions of the baseline signals. We establish the following empirical facts about machine learning for return prediction.

Machine learning shows great promise for empirical asset pricing. At the broadest level, our main empirical finding is that machine learning as a whole has the potential to improve our empirical understanding of expected asset returns. It digests our predictor data set, which is massive from the perspective of the existing literature, into a return forecasting model that dominates traditional approaches. The immediate implication is that machine learning aids in solving practical investments problems such as market timing, portfolio choice, and risk management, justifying its role in the business architecture of the fintech industry.

Consider as a benchmark a panel regression of individual stock returns onto three lagged stocklevel characteristics: size, book-to-market, and momentum. This benchmark has a number of attrac-

\footnotetext{
${ }^{3}$ See, for example, Hastie et al. (2009).

${ }^{4}$ See, for example, Jaggi (2013) and Hastie et al. (2009), who discuss the equivalence of support vector machines with the lasso. For an application of the kernel trick to the cross section of returns, see Kozak (2019).
} 
tive features. It is parsimonious and simple, and comparing against this benchmark is conservative because it is highly selected (the characteristics it includes are routinely demonstrated to be among the most robust return predictors). Lewellen (2015) demonstrates that this model performs about as well as larger and more complex stock prediction models studied in the literature.

In our sample, which is longer and wider (more observations in terms of both dates and stocks) than that studied in Lewellen (2015), the out-of-sample $R^{2}$ from the benchmark model is $0.16 \%$ per month for the panel of individual stock returns. When we expand the OLS panel model to include our set of $900+$ predictors, predictability vanishes immediately - the $R^{2}$ drops deeply into negative territory. This is not surprising. With so many parameters to estimate, efficiency of OLS regression deteriorates precipitously and therefore produces forecasts that are highly unstable out-of-sample. This failure of OLS leads us to our next empirical fact.

Vast predictor sets are viable for linear prediction when either penalization or dimension reduction is used. Our first evidence that the machine learning toolkit aids in return prediction emerges from the fact that the "elastic net," which uses parameter shrinkage and variable selection to limit the regression's degrees of freedom, solves the OLS inefficiency problem. In the 900+ predictor regression, elastic net pulls the out-of-sample $R^{2}$ into positive territory at $0.11 \%$ per month. Principal components regression (PCR) and partial least squares (PLS), which reduce the dimension of the predictor set to a few linear combinations of predictors, further raise the out-of-sample $R^{2}$ to $0.26 \%$ and $0.27 \%$, respectively. This is in spite of the presence of many likely "fluke" predictors that contribute pure noise to the large model. In other words, the high-dimensional predictor set in a simple linear specification is at least competitive with the status quo low-dimensional model, as long as over-parameterization can be controlled.

Allowing for nonlinearities substantially improves predictions. Next, we expand the model to accommodate nonlinear predictive relationships via generalized linear models, regression trees, and neural networks. We find that trees and neural networks unambiguously improve return prediction with monthly stock-level $R^{2}$ 's between $0.33 \%$ and $0.40 \%$. But the generalized linear model, which introduces nonlinearity via spline functions of each individual baseline predictor (but with no predictor interactions), fails to robustly outperform the linear specification. This suggests that allowing for (potentially complex) interactions among the baseline predictors is a crucial aspect of nonlinearities in the expected return function. As part of our analysis, we discuss why generalized linear models are comparatively poorly suited for capturing predictor interactions.

Shallow learning outperforms deeper learning. When we consider a range of neural networks from very shallow (a single hidden layer) to deeper networks (up to five hidden layers), we find that neural network performance peaks at three hidden layers then declines as more layers are added. Likewise, the boosted tree and random forest algorithms tend to select trees with few "leaves" (on average less than six leaves) in our analysis. This is likely an artifact of the relatively small amount of data and tiny signal-to-noise ratio for our return prediction problem, in comparison to the kinds of non-financial settings in which deep learning thrives thanks to astronomical datasets and strong signals (such as computer vision).

The distance between nonlinear methods and the benchmark widens when predicting portfolio 
returns. We build bottom-up portfolio-level return forecasts from the stock-level forecasts produced by our models. Consider, for example, bottom-up forecasts of the S\&P 500 portfolio return. By aggregating stock-level forecasts from the benchmark three-characteristic OLS model, we find a monthly S\&P 500 predictive $R^{2}$ of $-0.22 \%$. The bottom-up S\&P 500 forecast from the generalized linear model, in contrast, delivers an $R^{2}$ of $0.71 \%$. Trees and neural networks improve upon this further, generating monthly out-of-sample $R^{2}$ 's between $1.08 \%$ to $1.80 \%$ per month. The same pattern emerges for forecasting a variety of characteristic factor portfolios, such as those formed on the basis of size, value, investment, profitability, and momentum. In particular, a neural network with three layers produces a positive out-of-sample predictive $R^{2}$ for every factor portfolio we consider.

More pronounced predictive power at the portfolio level versus the stock level is driven by the fact that individual stock returns behave erratically for some of the smallest and least liquid stocks in our sample. Aggregating into portfolios averages out much of the unpredictable stock-level noise and boosts the signal strength, which helps in detecting the predictive gains from machine learning.

The economic gains from machine learning forecasts are large. Our tests show clear statistical rejections of the OLS benchmark and other linear models in favor of nonlinear machine learning tools. The evidence for economic gains from machine learning forecasts - in the form of portfolio Sharpe ratios - are likewise impressive. For example, an investor who times the S\&P 500 based on bottom-up neural network forecasts enjoys a 26 percentage point increase in annualized out-of-sample Sharpe ratio, to 0.77 , relative to the 0.51 Sharpe ratio of a buy-and-hold investor. And when we form a long-short decile spread directly sorted on stock return predictions from a neural network, the strategy earns an annualized out-of-sample Sharpe ratio of 1.35 (value-weighted) and 2.45 (equalweighted). In contrast, an analogous long-short strategy using forecasts from the benchmark OLS model delivers Sharpe ratios of 0.61 and 0.83 , respectively.

The most successful predictors are price trends, liquidity, and volatility. All of the methods we study produce a very similar ranking of the most informative stock-level predictors, which fall into three main categories. First, and most informative of all, are price trend variables including stock momentum, industry momentum, and short-term reversal. Next are liquidity variables including market value, dollar volume, and bid-ask spread. Finally, return volatility, idiosyncratic volatility, market beta, and beta squared are also among the leading predictors in all models we consider.

Better understanding our machine learning findings through simulation. In Appendix A we perform Monte Carlo simulations that support the above interpretations of our analysis. We apply machine learning to simulated data from two different data generating processes. Both produce data from a high dimensional predictor set. But in one, individual predictors enter only linearly and additively, while in the other predictors can enter through nonlinear transformations and via pairwise interactions. When we apply our machine learning repertoire to the simulated datasets, we find that linear and generalized linear methods dominate in the linear and uninteracted setting, yet tree-based methods and neural networks significantly outperform in the nonlinear and interactive setting. 


\subsection{What Machine Learning Cannot Do}

Machine learning has great potential for improving risk premium measurement, which is fundamentally a problem of prediction. It amounts to best approximating the conditional expectation $\mathrm{E}\left(r_{i, t+1} \mid \mathcal{F}_{t}\right)$, where $r_{i, t+1}$ is an asset's return in excess of the risk-free rate, and $\mathcal{F}_{t}$ is the true and unobservable information set of market participants. This is a domain in which machine learning algorithms excel.

But these improved predictions are only measurements. The measurements do not tell us about economic mechanisms or equilibria. Machine learning methods on their own do not identify deep fundamental associations among asset prices and conditioning variables. When the objective is to understand economic mechanisms, machine learning may still be useful. It requires the economist to add structure - to build a hypothesized mechanism into the estimation problem - and decide how to introduce a machine learning algorithm subject to this structure. A nascent literature has begun to make progress marrying machine learning with equilibrium asset pricing (for example, Kelly et al., 2019; Feng et al., 2019), and this remains an exciting direction for future research.

\subsection{Literature}

Our work extends the empirical literature on stock return prediction, which comes in two basic strands. The first strand models differences in expected returns across stocks as a function of stocklevel characteristics, and is exemplified by Fama and French (2008) and Lewellen (2015). The typical approach in this literature runs cross-sectional regressions ${ }^{5}$ of future stock returns on a few lagged stock characteristics. The second strand forecasts the time series of returns and is surveyed by Koijen and Nieuwerburgh (2011) and Rapach and Zhou (2013). This literature typically conducts time series regressions of broad aggregate portfolio returns on a small number of macroeconomic predictor variables.

These traditional methods have potentially severe limitations that more advanced statistical tools in machine learning can help overcome. Most important is that regressions and portfolio sorts are ill-suited to handle the large numbers of predictor variables that the literature has accumulated over five decades. The challenge is how to assess the incremental predictive content of a newly proposed predictor while jointly controlling for the gamut of extant signals (or, relatedly, handling the multiple comparisons and false discovery problem). Our primary contribution is to demonstrate potent return predictability that is harnessable from the large collection of existing variables when machine learning methods are used.

Machine learning methods have appeared sporadically in the asset pricing literature. Rapach et al. (2013) apply lasso to predict global equity market returns using lagged returns of all countries. Several papers apply neural-networks to forecast derivatives prices (Hutchinson et al., 1994; Yao et al., 2000, among others). Khandani et al. (2010) and Butaru et al. (2016) use regression trees to predict consumer credit card delinquencies and defaults. Sirignano et al. (2016) estimate a deep

\footnotetext{
${ }^{5}$ In addition to least squares regression, the literature often sorts assets into portfolios on the basis of characteristics and studies portfolio averages - a form of nonparametric regression.
} 
neural network for mortgage prepayment, delinquency, and foreclosure. Heaton et al. (2016) develop a neural network for portfolio selection.

Recently, variations of machine learning methods have been used to study the cross section of stock returns. Harvey and Liu (2016) study the multiple comparisons problem using a bootstrap procedure. Giglio and Xiu (2016) and Kelly et al. (2019) use dimension reduction methods to estimate and test factor pricing models. Moritz and Zimmermann (2016) apply tree-based models to portfolio sorting. Kozak et al. (2019) and Freyberger et al. (2019) use shrinkage and selection methods to, respectively, approximate a stochastic discount factor and a nonlinear function for expected returns. The focus of our paper is to simultaneously explore a wide range of machine learning methods to study the behavior of expected stock returns, with a particular emphasis on comparative analysis among methods.

\section{Methodology}

This section describes the collection of machine learning methods that we use in our analysis. In each subsection we introduce a new method and describe it in terms of its three fundamental elements. First is the statistical model describing a method's general functional form for risk premium predictions. The second is an objective function for estimating model parameters. All of our estimates share the basic objective of minimizing mean squared predictions error (MSE). Regularization is introduced through variations on the MSE objective, such as adding parameterization penalties and robustification against outliers. These modifications are designed to avoid problems with overfit and improve models' out-of-sample predictive performance. Finally, even with a small number of predictors, the set of model permutations expands rapidly when one considers nonlinear predictor transformations. This proliferation is compounded in our already high dimension predictor set. The third element in each subsection describes computational algorithms for efficiently identifying the optimal specification among the permutations encompassed by a given method.

As we present each method, we aim to provide a sufficiently in-depth description of the statistical model so that a reader having no machine learning background can understand the basic model structure without needing to consult outside sources. At the same time, when discussing the computational methods for estimating each model, we are deliberately terse. There are many variants of each algorithm, and each has its own subtle technical nuances. To avoid bogging down the reader with programming details, we describe our specific implementation choices in Appendix B and refer readers to original sources for further background. We also summarize the literature on statistical properties of each estimator in Appendix C.

In its most general form, we describe an asset's excess return as an additive prediction error model:

$$
r_{i, t+1}=\mathrm{E}_{t}\left(r_{i, t+1}\right)+\epsilon_{i, t+1},
$$

where

$$
\mathrm{E}_{t}\left(r_{i, t+1}\right)=g^{\star}\left(z_{i, t}\right) .
$$


Stocks are indexed as $i=1, \ldots, N_{t}$ and months by $t=1, \ldots, T$. For ease of presentation, we assume a balanced panel of stocks, and defer the discussion on missing data to Section 3.1. Our objective is to isolate a representation of $\mathrm{E}_{t}\left(r_{i, t+1}\right)$ as a function of predictor variables that maximizes the outof-sample explanatory power for realized $r_{i, t+1}$. We denote those predictors as the $P$-dimensional vector $z_{i, t}$, and assume the conditional expected return $g^{\star}(\cdot)$ is a flexible function of these predictors.

Despite its flexibility, this framework imposes some important restrictions. The $g^{\star}(\cdot)$ function depends neither on $i$ nor $t$. By maintaining the same form over time and across different stocks, the model leverages information from the entire panel which lends stability to estimates of risk premia for any individual asset. This is in contrast to standard asset pricing approaches that re-estimate a cross-sectional model each time period, or that independently estimate time series models for each stock. Also, $g^{\star}(\cdot)$ depends on $z$ only through $z_{i, t}$. This means our prediction does not use information from the history prior to $t$, or from individual stocks other than the $i^{\text {th }}$.

\subsection{Sample Splitting and Tuning via Validation}

Important preliminary steps (prior to discussing specific models and regularization approaches) are to understand how we design disjoint sub-samples for estimation and testing and to introduce the notion of "hyperparameter tuning."

The regularization procedures discussed below, which are machine learning's primary defense against overfitting, rely on a choice of hyperparameters (or, synonymously, "tuning parameters"). These are critical to the performance of machine learning methods as they control model complexity. Hyperparameters include, for example, the penalization parameters in lasso and elastic net, the number of iterated trees in boosting, the number of random trees in a forest, and the depth of the trees. In most cases, there is little theoretical guidance for how to "tune" hyperparameters for optimized out-of-sample performance. ${ }^{6}$

We follow the most common approach in the literature and select tuning parameters adaptively from the data in a validation sample. In particular, we divide our sample into three disjoint time periods that maintain the temporal ordering of the data. The first, or "training," subsample is used to estimate the model subject to a specific set of tuning parameter values.

The second, or "validation," sample is used for tuning the hyperparameters. We construct forecasts for data points in the validation sample based on the estimated model from the training sample. Next, we calculate the objective function based on forecast errors from the validation sample, and iteratively search for hyperparameters that optimize the validation objective (at each step re-estimating the model from the training data subject to the prevailing hyperparameter values).

Tuning parameters are chosen from the validation sample taking into account estimated parameters, but the parameters are estimated from the training data alone. The idea of validation is to simulate an out-of-sample test of the model. Hyperparameter tuning amounts to searching for a degree of model complexity that tends to produce reliable out-of-sample performance. The validation sample fits are of course not truly out-of-sample because they are used for tuning, which is in

\footnotetext{
${ }^{6}$ In machine learning, a "hyperparameter" governs the extent of estimator regularization. This usage is related to, but different from, its meaning in Bayesian statistics as a parameter of a prior distribution.
} 
turn an input to the estimation. Thus the third, or "testing," subsample, which is used for neither estimation nor tuning, is truly out-of-sample and thus is used to evaluate a method's predictive performance. Further details of our sample splitting scheme are provided in Appendix D, and a summary of hyperparameter tuning schemes for each model is provided in Appendix E.

\subsection{Simple Linear}

We begin our model description with the least complex method in our analysis, the simple linear predictive regression model estimated via ordinary least squares (OLS). While we expect this to perform poorly in our high dimension problem, we use it as a reference point for emphasizing the distinctive features of more sophisticated methods.

Model. The simple linear model imposes that conditional expectations $g^{\star}(\cdot)$ can be approximated by a linear function of the raw predictor variables and the parameter vector, $\theta$,

$$
g\left(z_{i, t} ; \theta\right)=z_{i, t}^{\prime} \theta .
$$

This model imposes a simple regression specification and does not allow for nonlinear effects or interactions between predictors.

Objective Function and Computational Algorithm. Our baseline estimation of the simple linear model uses a standard least squares, or " $l_{2}$ ", objective function:

$$
\mathcal{L}(\theta)=\frac{1}{N T} \sum_{i=1}^{N} \sum_{t=1}^{T}\left(r_{i, t+1}-g\left(z_{i, t} ; \theta\right)\right)^{2} .
$$

Minimizing $\mathcal{L}(\theta)$ yields the pooled OLS estimator. The convenience of the baseline $l_{2}$ objective function is that it offers analytical estimates and thus avoids sophisticated optimization and computation.

\subsubsection{Extension: Robust Objective Functions}

In some cases it is possible to improve predictive performance by replacing equation (4) with a weighted least squares objective such as

$$
\mathcal{L}_{W}(\theta)=\frac{1}{N T} \sum_{i=1}^{N} \sum_{t=1}^{T} w_{i, t}\left(r_{i, t+1}-g\left(z_{i, t} ; \theta\right)\right)^{2} .
$$

This allows the econometrician to tilt estimates towards observations that are more statistically or economically informative. For example, one variation that we consider sets $w_{i, t}$ inversely proportional to the number of stocks at time $t$. This imposes that every month has the same contribution to the model regardless of how many stocks are available that month. This also amounts to equally weighting the squared loss of all stocks available at time $t$. Another variation that we consider sets $w_{i, t}$ proportional to the equity market value of stock $i$ at time $t$. This value weighted loss function underweights small stocks in favor of large stocks, and is motivated by the economic rational that 
small stocks represent a large fraction of the traded universe by count while constituting a tiny fraction of aggregate market capitalization. ${ }^{7}$

Heavy tails are a well known attribute of financial returns and stock-level predictor variables. Convexity of the least squares objective (4) places extreme emphasis on large errors, thus outliers can undermine the stability of OLS-based predictions. The statistics literature, long aware of this problem, has developed modified least squares objective functions that tend to produce more stable forecasts than OLS in the presence of extreme observations. ${ }^{8}$ In the machine learning literature, a common choice for counteracting the deleterious effect of heavy-tailed observations is the Huber robust objective function, defined as

$$
\mathcal{L}_{H}(\theta)=\frac{1}{N T} \sum_{i=1}^{N} \sum_{t=1}^{T} H\left(r_{i, t+1}-g\left(z_{i, t} ; \theta\right), \xi\right),
$$

where

$$
H(x ; \xi)=\left\{\begin{array}{lll}
x^{2}, & \text { if } & |x| \leq \xi ; \\
2 \xi|x|-\xi^{2}, & \text { if } & |x|>\xi .
\end{array} .\right.
$$

The Huber loss, $H(\cdot)$, is a hybrid of squared loss for relatively small errors and absolute loss for relatively large errors, where the combination is controlled by a tuning parameter, $\xi$, that can be optimized adaptively from the data. ${ }^{9}$

While this detour introduces robust objective functions in the context of the simple linear model, they are easily applicable in almost all of the methods that we study. In our empirical analysis we study the predictive benefits of robust loss functions in multiple machine learning methods.

\subsection{Penalized Linear}

The simple linear model is bound to fail in the presence of many predictors. When the number of predictors $P$ approaches the number of observations $T$, the linear model becomes inefficient or even inconsistent. ${ }^{10}$ It begins to overfit noise rather than extracting signal. This is particularly troublesome for the problem of return prediction where the signal-to-noise ratio is notoriously low.

Crucial for avoiding overfit is reducing the number of estimated parameters. The most common machine learning device for imposing parameter parsimony is to append a penalty to the objective function in order to favor more parsimonious specifications. This "regularization" of the estimation problem mechanically deteriorates a model's in-sample performance in hopes that it improves its stability out-of-sample. This will be the case when penalization manages to reduce the model's fit

\footnotetext{
${ }^{7}$ As of Fama and French (2008), the smallest 20\% of stocks comprise only $3 \%$ of aggregate market capitalization. An example of a statistically motivated weighting scheme uses $w_{i, t}$ inversely proportional to an observation's estimated error variance, a choice that potentially improves prediction efficiency in the spirit of generalized least squares.

${ }^{8}$ Classical analyses include Box (1953), Tukey (1960), and Huber (1964).

${ }^{9}$ OLS is a special case of the (6) with $\xi=\infty$. While most theoretical analysis in high-dimensional statistics assume that data have sub-Gaussian or sub-exponential tails, Fan et al. (2017) provide a theoretical justification of using this loss function in the high-dimensional setting as well as a procedure to determine the hyperparameter.

${ }^{10}$ We deliberately compare $P$ with $T$, instead of with $N T$, because stock returns share strong cross-sectional dependence, limiting the incremental information contained in new cross-section observations.
} 
of noise while preserving its fit of the signal.

Objective Function and Computational Algorithm. The statistical model for our penalized linear model is the same as the simple linear model in equation (3). That is, it continues to consider only the baseline, untransformed predictors. Penalized methods differ by appending a penalty to the original loss function:

$$
\mathcal{L}(\theta ; \cdot)=\mathcal{L}(\theta)+\phi(\theta ; \cdot) .
$$

There are several choices for the penalty function $\phi(\theta ; \cdot)$. We focus on the popular "elastic net" penalty, which takes the form

$$
\phi(\theta ; \lambda, \rho)=\lambda(1-\rho) \sum_{j=1}^{P}\left|\theta_{j}\right|+\frac{1}{2} \lambda \rho \sum_{j=1}^{P} \theta_{j}^{2} .
$$

The elastic net involves two non-negative hyperparameters, $\lambda$ and $\rho$, and includes two well known regularizers as special cases. The $\rho=0$ case corresponds to the lasso and uses an absolute value, or " $l_{1}$ ", parameter penalization. The fortunate geometry of the lasso sets coefficients on a subset of covariates to exactly zero. In this sense, the lasso imposes sparsity on the specification and can thus be thought of as a variable selection method. The $\rho=1$ case corresponds to ridge regression, which uses an $l_{2}$ parameter penalization, that draws all coefficient estimates closer to zero but does not impose exact zeros anywhere. In this sense, ridge is a shrinkage method that helps prevent coefficients from becoming unduly large in magnitude. For intermediate values of $\rho$, the elastic net encourages simple models through both shrinkage and selection.

We adaptively optimize the tuning parameters, $\lambda$ and $\rho$, using the validation sample. Our implementation of penalized regression uses the accelerated proximal gradient algorithm and accommodates both least squares and Huber objective functions (see Appendix B.1 for more detail).

\subsection{Dimension Reduction: PCR and PLS}

Penalized linear models use shrinkage and variable selection to manage high dimensionality by forcing the coefficients on most regressors near or exactly to zero. This can produce suboptimal forecasts when predictors are highly correlated. A simple example of this problem is a case in which all of the predictors are equal to the forecast target plus an iid noise term. In this situation, choosing a subset of predictors via lasso penalty is inferior to taking a simple average of the predictors and using this as the sole predictor in a univariate regression.

The idea of predictor averaging, as opposed to predictor selection, is the essence of dimension reduction. Forming linear combinations of predictors helps reduce noise to better isolate the signal in predictors, and also helps de-correlate otherwise highly dependent predictors. Two classic dimension reduction techniques are principal components regression (PCR) and partial least squares (PLS).

PCR consists of a two-step procedure. In the first step, principal components analysis (PCA) combines regressors into a small set of linear combinations that best preserve the covariance structure 
among the predictors. In the second step, a few leading components are used in standard predictive regression. That is, PCR regularizes the prediction problem by zeroing out coefficients on low variance components.

A drawback of PCR is that it fails to incorporate the ultimate statistical objective - forecasting returns - in the dimension reduction step. PCA condenses data into components based on the covariation among the predictors. This happens prior to the forecasting step and without consideration of how predictors associate with future returns.

In contrast, partial least squares performs dimension reduction by directly exploiting covariation of predictors with the forecast target. ${ }^{11}$ PLS regression proceeds as follows. For each predictor $j$, estimate its univariate return prediction coefficient via OLS. This coefficient, denoted $\varphi_{j}$, reflects the "partial" sensitivity of returns to each predictor $j$. Next, average all predictors into a single aggregate component with weights proportional to $\varphi_{j}$, placing the highest weight on the strongest univariate predictors, and the least weight on the weakest. In this way, PLS performs its dimension reduction with the ultimate forecasting objective in mind. To form more than one predictive component, the target and all predictors are orthogonalized with respect to previously constructed components, and the above procedure is repeated on the orthogonalized dataset. This is iterated until the desired number of PLS components is reached.

Model. Our implementation of PCR and PLS begins from the vectorized version of the linear model in equations (1)-(3). In particular, we reorganize the linear regression $r_{i, t+1}=z_{i, t}^{\prime} \theta+\epsilon_{i, t+1}$ as

$$
R=Z \theta+E,
$$

where $R$ is the $N T \times 1$ vector of $r_{i, t+1}, Z$ is the $N T \times P$ matrix of stacked predictors $z_{i, t}$, and $E$ is a $N T \times 1$ vector of residuals $\epsilon_{i, t+1}$.

PCR and PLS take the same general approach to reducing the dimensionality. They both condense the set of predictors from dimension $P$ to a much smaller number of $K$ linear combinations of predictors. Thus, the forecasting model for both methods is written as

$$
R=\left(Z \Omega_{K}\right) \theta_{K}+\tilde{E}
$$

$\Omega_{K}$ is $P \times K$ matrix with columns $w_{1}, w_{2}, \ldots, w_{K}$. Each $w_{j}$ is the set of linear combination weights used to create the $j^{\text {th }}$ predictive components, thus $Z \Omega_{K}$ is the dimension-reduced version of the original predictor set. Likewise, the predictive coefficient $\theta_{K}$ is now a $K \times 1$ vector rather than $P \times 1$.

Objective Function and Computational Algorithm. PCR chooses the combination weights $\Omega_{K}$ recursively. The $j^{\text {th }}$ linear combination solves ${ }^{12}$

$$
w_{j}=\arg \max _{w} \operatorname{Var}(Z w), \quad \text { s.t. } \quad w^{\prime} w=1, \quad \operatorname{Cov}\left(Z w, Z w_{l}\right)=0, \quad l=1,2, \ldots, j-1 .
$$

\footnotetext{
${ }^{11}$ See Kelly and Pruitt $(2013,2015)$ for asymptotic theory of PLS regression and its application to forecasting risk premia in financial markets.

${ }^{12}$ For two vectors $a$ and $b$, we denote $\operatorname{Cov}(a, b):=(a-\bar{a})^{\top}(b-\bar{b})$, where $\bar{a}$ is the average of all entries of $a$. Naturally, we define $\operatorname{Var}(a):=\operatorname{Cov}(a, a)$.
} 
Intuitively, PCR seeks the $K$ linear combinations of $Z$ that most faithfully mimic the full predictor set. The objective illustrates that the choice of components is not based on the forecasting objective at all. Instead, the emphasis of PCR is on finding components that retain the most possible common variation within the predictor set. The well known solution for (11) computes $\Omega_{K}$ via singular value decomposition of $Z$, and therefore the PCR algorithm is extremely efficient from a computational standpoint.

In contrast to PCR, the PLS objective seeks $K$ linear combinations of $Z$ that have maximal predictive association with the forecast target. The weights used to construct $j^{\text {th }}$ PLS component solve

$$
w_{j}=\arg \max _{w} \operatorname{Cov}^{2}(R, Z w), \quad \text { s.t. } \quad w^{\prime} w=1, \quad \operatorname{Cov}\left(Z w, Z w_{l}\right)=0, \quad l=1,2, \ldots, j-1 .
$$

This objective highlights the main distinction between PCR and PLS. PLS is willing to sacrifice how accurately $Z \Omega_{K}$ approximates $Z$ in order to find components with more potent return predictability. The problem in (12) can be efficiently solved using a number of similar routines, the most prominent being the SIMPLS algorithm of de Jong (1993).

Finally, given a solution for $\Omega_{K}, \theta_{K}$ is estimated in both PCR and PLS via OLS regression of $R$ on $Z \Omega_{K}$. For both models, $K$ is a hyperparameter that can be determined adaptively from the validation sample.

\subsection{Generalized Linear}

Linear models are popular in practice, in part because they can be thought of as a first-order approximation to the data generating process. ${ }^{13}$ When the "true" model is complex and nonlinear, restricting the functional form to be linear introduces approximation error due to model misspecification. Let $g^{\star}\left(z_{i, t}\right)$ denote the true model and $g\left(z_{i, t} ; \theta\right)$ the functional form specified by the econometrician. And let $g\left(z_{i, t} ; \widehat{\theta}\right)$ and $\widehat{r}_{i, t+1}$ denote the fitted model and its ensuing return forecast. We can decompose a model's forecast error as:

$$
r_{i, t+1}-\widehat{r}_{i, t+1}=\underbrace{g^{\star}\left(z_{i, t}\right)-g\left(z_{i, t} ; \theta\right)}_{\text {approximation error }}+\underbrace{g\left(z_{i, t} ; \theta\right)-g\left(z_{i, t} ; \widehat{\theta}\right)}_{\text {estimation error }}+\underbrace{\epsilon_{i, t+1}}_{\text {intrinsic error }} .
$$

Intrinsic error is irreducible; it is the genuinely unpredictable component of returns associated with news arrival and other sources of randomness in financial markets. Estimation error, which arises due to sampling variation, is determined by the data. It is potentially reducible by adding new observations, though this may not be under the econometrician's control. Approximation error is directly controlled by the econometrician, and is potentially reducible by incorporating more flexible specifications that improve the model's ability to approximate the true model. But additional flexibility raises the risk of overfitting and destabilizing the model out-of-sample. In this and the following subsections, we introduce nonparametric models of $g(\cdot)$ with increasing degrees of flexibility,

\footnotetext{
${ }^{13}$ See White (1980) for a discussion of limitations of linear models as first-order approximations.
} 
each complemented by regularization methods to mitigate overfit.

Model. The first and most straightforward nonparametric approach that we consider is the generalized linear model. It introduces nonlinear transformations of the original predictors as new additive terms in an otherwise linear model. Generalized linear models are thus the closest nonlinear counterparts to the linear approaches in Sections 2.2 and 2.3.

The model we study adapts the simple linear form by adding a $K$-term spline series expansion of the predictors

$$
g(z ; \theta, p(\cdot))=\sum_{j=1}^{P} p\left(z_{j}\right)^{\prime} \theta_{j},
$$

where $p(\cdot)=\left(p_{1}(\cdot), p_{2}(\cdot), \ldots, p_{K}(\cdot)\right)^{\prime}$ is a vector of basis functions, and the parameters are now a $K \times N$ matrix $\theta=\left(\theta_{1}, \theta_{2}, \ldots, \theta_{N}\right)$. There are many potential choices for spline functions. We adopt a spline series of order two: $\left(1, z,\left(z-c_{1}\right)^{2},\left(z-c_{2}\right)^{2}, \ldots,\left(z-c_{K-2}\right)^{2}\right)$, where $c_{1}, c_{2}, \ldots c_{K-2}$ are knots.

Objective Function and Computational Algorithm. Because higher order terms enter additively, forecasting with the generalized linear model can be approached with the same estimation tools as in Section 2.2. In particular, our analysis uses a least squares objective function, both with and without the Huber robustness modification. Because series expansion quickly multiplies the number of model parameters, we use penalization to control degrees of freedom. Our choice of penalization function is specialized for the spline expansion setting and is known as the group lasso. It takes the form

$$
\phi(\theta ; \lambda, K)=\lambda \sum_{j=1}^{P}\left(\sum_{k=1}^{K} \theta_{j, k}^{2}\right)^{1 / 2} .
$$

As its name suggests, the group lasso selects either all $K$ spline terms associated with a given characteristic or none of them. We embed this penalty in the general objective of equation (7). Group lasso accommodates either least squares or robust Huber objective, and it uses the same accelerated proximal gradient descent as the elastic net. It has two tuning parameters, $\lambda$ and $K .{ }^{14}$

\subsection{Boosted Regression Trees and Random Forests}

The model in (13) captures individual predictors' nonlinear impact on expected returns, but does not account for interactions among predictors. One way to add interactions is to expand the generalized model to include multivariate functions of predictors. While expanding univariate predictors with $K$ basis functions multiplies the number of parameters by a factor of $K$, multi-way interactions increase the parameterization combinatorially. Without a priori assumptions for which interactions to include, the generalized linear model becomes computationally infeasible. ${ }^{15}$

\footnotetext{
${ }^{14}$ For additional details, see Appendix B.1. A similar model in the return prediction context is Freyberger et al. (2019).

${ }^{15}$ Parameter penalization does not solve the difficulty of estimating linear models when the number of predictors is exponentially larger than the number of observations. Instead, one must turn to heuristic optimization algorithms such as stepwise regression (sequentially adding/dropping variables until some stopping rule is satisfied), variable screening (retaining predictors whose univariate correlations with the prediction target exceed a certain value), or others.
} 
Figure 1: Regression Tree Example
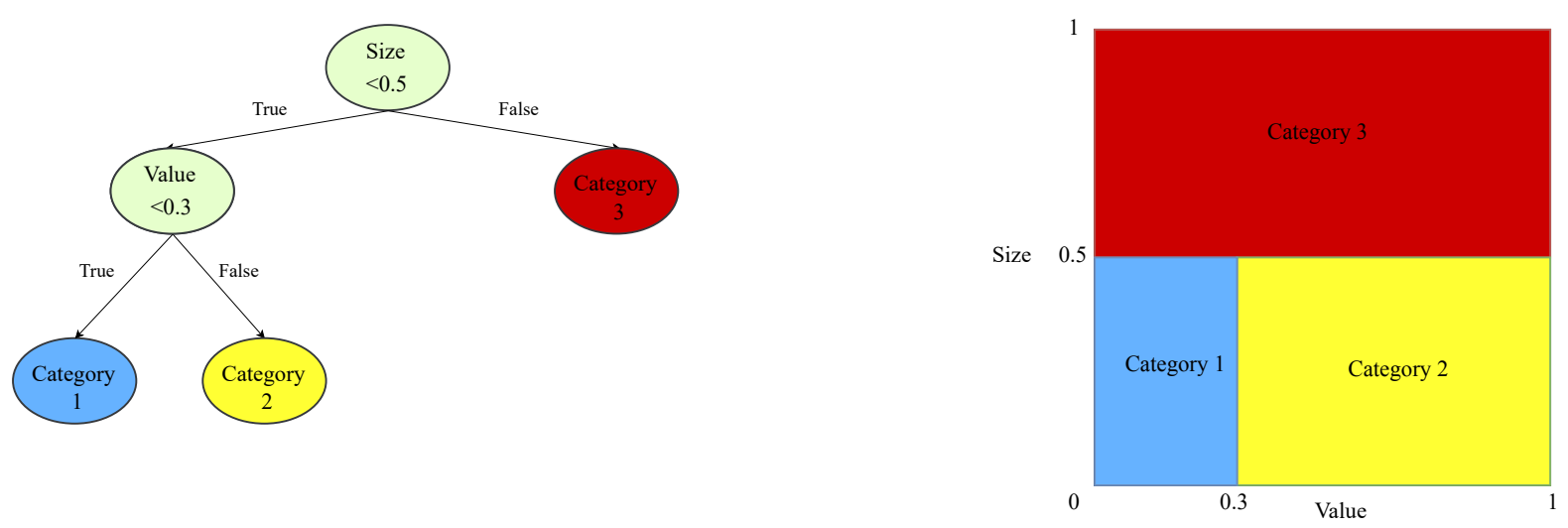

Note: This figure presents the diagrams of a regression tree (left) and its equivalent representation (right) in the space of two characteristics (size and value). The terminal nodes of the tree are colored in blue, yellow, and red, respectively. Based on their values of these two characteristics, the sample of individual stocks is divided into three categories.

As an alternative, regression trees have become a popular machine learning approach for incorporating multi-way predictor interactions. Unlike linear models, trees are fully nonparametric and possess a logic that departs markedly from traditional regressions. At a basic level, trees are designed to find groups of observations that behave similarly to each. A tree "grows" in a sequence of steps. At each step, a new "branch" sorts the data leftover from the preceding step into bins based on one of the predictor variables. This sequential branching slices the space of predictors into rectangular partitions, and approximates the unknown function $g^{\star}(\cdot)$ with the average value of the outcome variable within each partition.

Figure 1 shows an example with two predictors, "size" and "b/m." The left panel describes how the tree assigns each observation to a partition based on its predictor values. First, observations are sorted on size. Those above the breakpoint of 0.5 are assigned to Category 3. Those with small size are then further sorted by $\mathrm{b} / \mathrm{m}$. Observations with small size and $\mathrm{b} / \mathrm{m}$ below 0.3 are assigned to Category 1, while those with b/m above 0.3 go into Category 2. Finally, forecasts for observations in each partition are defined as the simple average of the outcome variable's value among observations in that partition.

Model. More formally, the prediction of a tree, $\mathcal{T}$, with $K$ "leaves" (terminal nodes), and depth $L$, can be written as

$$
g\left(z_{i, t} ; \theta, K, L\right)=\sum_{k=1}^{K} \theta_{k} \mathbf{1}_{\left\{z_{i, t} \in C_{k}(L)\right\}}
$$

where $C_{k}(L)$ is one of the $K$ partitions of the data. Each partition is a product of up to $L$ indicator functions of the predictors. The constant associated with partition $k$ (denoted $\theta_{k}$ ) is defined to be 
the sample average of outcomes within the partition. ${ }^{16}$ In the example of Figure 1, the prediction equation is

$$
g\left(z_{i, t} ; \theta, 3,2\right)=\theta_{1} \mathbf{1}_{\left\{\operatorname{size}_{i, t}<0.5\right\}} \mathbf{1}_{\left\{\mathrm{b} / \mathrm{m}_{i, t}<0.3\right\}}+\theta_{2} \mathbf{1}_{\left\{\mathrm{size}_{i, t}<0.5\right\}} \mathbf{1}_{\left\{\mathrm{b} / \mathrm{m}_{i, t} \geq 0.3\right\}}+\theta_{3} \mathbf{1}_{\left\{\operatorname{size}_{i, t} \geq 0.5\right\}} .
$$

Objective Function and Computational Algorithm. To grow a tree is to find bins that best discriminate among the potential outcomes. The specific predictor variable upon which a branch is based, and the specific value where the branch is split, is chosen to minimize forecast error. The expanse of potential tree structures, however, precludes exact optimization. The literature has developed a set of sophisticated optimization heuristics to quickly converge on approximately optimal trees. We follow the algorithm of Breiman et al. (1984), which we describe in detail in Appendix B.2. The basic idea is to myopically optimize forecast error at the start of each branch. At each new level, we choose a sorting variable from the set of predictors and the split value to maximize the discrepancy among average outcomes in each bin. ${ }^{17}$ The loss associated with the forecast error for a branch $C$ is often called "impurity," which describes how similarly observations behave on either side of the split. We choose the most popular $l_{2}$ impurity for each branch of the tree:

$$
H(\theta, C)=\frac{1}{|C|} \sum_{z_{i, t} \in C}\left(r_{i, t+1}-\theta\right)^{2},
$$

where $|C|$ denotes the number of observations in set $C$. Given $C$, it is clear that the optimal choice of $\theta: \theta=\frac{1}{|C|} \sum_{z_{i, t} \in C} r_{i, t+1}$. The procedure is equivalent to finding the branch $C$ that locally minimizes the impurity. Branching halts when the number of leaves or the depth of the tree reach a pre-specified threshold that can be selected adaptively using a validation sample.

Among the advantages of a tree model are that it is invariant to monotonic transformations of predictors, that it naturally accommodates categorical and numerical data in the same model, that it can approximate potentially severe nonlinearities, and that a tree of depth $L$ can capture $(L-1)$ way interactions. Their flexibility is also their limitation. Trees are among the prediction methods most prone to overfit, and therefore must be heavily regularized. In our analysis, we consider two "ensemble" tree regularizers that combine forecasts from many different trees into a single forecast. ${ }^{18}$

Boosting. The first regularization method is "boosting," which recursively combines forecasts from many over-simplified trees. ${ }^{19}$ Shallow trees on their own are "weak learners" with minuscule predictive power. The theory behind boosting suggests that many weak learners may, as an ensemble, comprise a single "strong learner" with greater stability than a single complex tree.

\footnotetext{
${ }^{16}$ We focus on recursive binary trees for their relative simplicity. Breiman et al. (1984) discuss more complex tree structures.

${ }^{17}$ Because splits are chosen without consideration of future potential branches, it is possible to myopically bypass an inferior branch that would have led to a future branch with an ultimately superior reduction in forecast error.

${ }^{18}$ The literature also considers a number of other approaches to tree regularization such as early stopping and postpruning, both of which are designed to reduce overfit in a single large tree. Ensemble methods demonstrate more reliable performance and are scalable for very large datasets, leading to their increased popularity in recent literature.

${ }^{19}$ Boosting is originally described in Schapire (1990) and Freund (1995) for classification problems to improve the performance of a set of weak learners. Friedman et al. (2000a) and Friedman (2001) extend boosting to contexts beyond classification, eventually leading to the gradient boosted regression tree.
} 
The details of our boosting procedure, typically referred to as gradient boosted regression trees (GBRT), are described in Algorithm 4 of Appendix B.2. It starts by fitting a shallow tree (e.g., with depth $L=1$ ). This over-simplified tree is sure to be a weak predictor with large bias in the training sample. Next, a second simple tree (with the same shallow depth $L$ ) is used to fit the prediction residuals from the first tree. Forecasts from these two trees are added together to form an ensemble prediction of the outcome, but the forecast component from the second tree is shrunken by a factor $\nu \in(0,1)$ to help prevent the model from overfitting the residuals. At each new step $b$, a shallow tree is fitted to the residuals from the model with $b-1$ trees, and its residual forecast is added to the total with a shrinkage weight of $\nu$. This is iterated until there are a total of $B$ trees in the ensemble. The final output is therefore an additive model of shallow trees with three tuning parameters $(L, \nu, B)$ which we adaptively choose in the validation step.

Random Forest. Like boosting, a random forest is an ensemble method that combines forecasts from many different trees. It is a variation on a more general procedure known as bootstrap aggregation, or "bagging" (Breiman, 2001). The baseline tree bagging procedure draws $B$ different bootstrap samples of the data, fits a separate regression tree to each, then averages their forecasts. Trees for individual bootstrap samples tend to be deep and overfit, making their individual predictions inefficiently variable. Averaging over multiple predictions reduces this variation, thus stabilizing the trees' predictive performance.

Random forests use a variation on bagging designed to reduce the correlation among trees in different bootstrap samples. If, for example, firm size is the dominant return predictor in the data, then most of the bagged trees will have low-level splits on size resulting in substantial correlation among their ultimate predictions. The forest method de-correlates trees using a method known as "dropout," which considers only a randomly drawn subset of predictors for splitting at each potential branch. Doing so ensures that, in the example, early branches for at least a few trees will split on characteristics other than firm size. This lowers the average correlation among predictions to further improve the variance reduction relative to standard bagging. Depth $L$ of the trees and number of bootstrap samples $B$ are the tuning parameters optimized via validation. Precise details of our random forest implementation are described in Algorithm 3 of the appendix.

\subsection{Neural Networks}

The final nonlinear method that we analyze is the artificial neural network. Arguably the most powerful modeling device in machine learning, neural networks have theoretical underpinnings as "universal approximators" for any smooth predictive association (Hornik et al., 1989; Cybenko, 1989). They are the currently preferred approach for complex machine learning problems such as computer vision, natural language processing, and automated game-playing (such as chess and go). Their flexibility draws from the ability to entwine many telescoping layers of nonlinear predictor interactions, earning the synonym "deep learning." At the same time, their complexity ranks neural networks among the least transparent, least interpretable, and most highly parameterized machine learning tools. 
Figure 2: Neural Networks
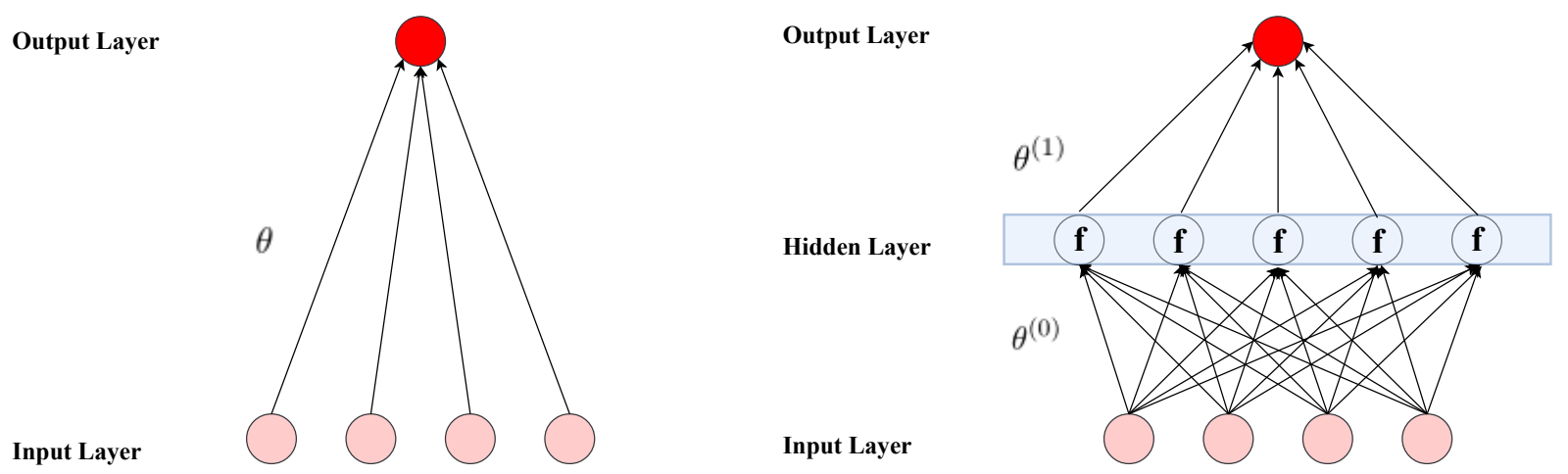

Note: This figure provides diagrams of two simple neural networks with (right) or without (left) a hidden layer. Pink circles denote the input layer and dark red circles denote the output layer. Each arrow is associated with a weight parameter. In the network with a hidden layer, a nonlinear activation function $f$ transforms the inputs before passing them on to the output.

Model. We focus our analysis on traditional "feed-forward" networks. These consist of an "input layer" of raw predictors, one or more "hidden layers" that interact and nonlinearly transform the predictors, and an "output layer" that aggregates hidden layers into an ultimate outcome prediction. Analogous to axons in a biological brain, layers of the networks represent groups of "neurons" with each layer connected by "synapses" that transmit signals among neurons of different layers. Figure 2 shows two illustrative examples.

The number of units in the input layer is equal to the dimension of the predictors, which we set to four in this example (denoted $z_{1}, \ldots, z_{4}$ ). The left panel shows the simplest possible network that has no hidden layers. Each of the predictor signals is amplified or attenuated according to a five-dimensional parameter vector, $\theta$, that includes an intercept and one weight parameter per predictor. The output layer aggregates the weighted signals into the forecast $\theta_{0}+\sum_{k=1}^{4} z_{k} \theta_{k}$; that is, the simplest neural network is a linear regression model.

The model incorporates more flexible predictive associations by adding hidden layers between the inputs and output. The right panel of Figure 2 shows an example with one hidden layer that contains five neurons. Each neuron draws information linearly from all of the input units, just as in the simple network on the left. Then, each neuron applies a nonlinear "activation function" $f$ to its aggregated signal before sending its output to the next layer. For example, the second neuron in the hidden layer transforms inputs into an output as $x_{2}^{(1)}=f\left(\theta_{2,0}^{(0)}+\sum_{j=1}^{4} z_{j} \theta_{2, j}^{(0)}\right)$. Lastly, the results from each neuron are linearly aggregated into an ultimate output forecast:

$$
g(z ; \theta)=\theta_{0}^{(1)}+\sum_{j=1}^{5} x_{j}^{(1)} \theta_{j}^{(1)} .
$$

Thus, in this example, there are a total of $31=(4+1) \times 5+6$ parameters (five parameters to reach 
each neuron and six weights to aggregate the neurons into a single output).

There are many choices to make when structuring a neural network, including the number of hidden layers, the number of neurons in each layer, and which units are connected. Despite the aforementioned "universal approximation" result that suggests the sufficiency of a single hidden layer, recent literature has shown that deeper networks can often achieve the same accuracy with substantially fewer parameters. ${ }^{20}$

On the other hand, in small data sets simple networks with only a few layers and nodes often perform best. Training a very deep neural network is challenging because it typically involves a large number of parameters, because the objective function is highly non-convex, and because the recursive calculation of derivatives (known as "back-propagation") is prone to exploding or vanishing gradients.

Selecting a successful network architecture by cross-validation is in general a difficult task. It is unrealistic and unnecessary to find the optimal network by searching over uncountably many architectures. Instead, we fix a variety of network architectures ex ante and estimate each of these. What we hope to achieve is reasonably lower bound on the performance of machine learning methods.

We consider architectures with up to five hidden layers. Our shallowest neural network has a single hidden layer of 32 neurons, which we denoted NN1. Next, NN2 has two hidden layers with 32 and 16 neurons, respectively; NN3 has three hidden layers with 32, 16, and 8 neurons, respectively; NN4 has four hidden layers with 32, 16, 8, 4 neurons, respectively; and NN5 has five hidden layers with $32,16,8,4$, and 2 neurons, respectively. We choose the number of neurons in each layer according to the geometric pyramid rule (see Masters, 1993). All architectures are fully connected so each unit receives an input from all units in the layer below. By comparing the performance of NN1 through NN5, we can infer the trade-offs of network depth in the return forecasting problem. ${ }^{21}$

There are many potential choices for the nonlinear activation function (such as sigmoid, hyperbolic, softmax, etc.). We use the same activation function at all nodes, and choose a popular functional form in recent literature known as the rectified linear unit (ReLU), defined $a^{22}$

$$
\operatorname{ReLU}(x)=\left\{\begin{array}{lc}
0 & \text { if } x<0 \\
x & \text { otherwise }
\end{array}\right.
$$

which encourages sparsity in the number of active neurons, and allows for faster derivative evaluation.

Our neural network model has the following general formula. Let $K^{(l)}$ denote the number of neurons in each layer $l=1, \ldots, L$. Define the output of neuron $k$ in layer $l$ as $x_{k}^{(l)}$. Next, define the vector of outputs for this layer (augmented to include a constant, $\left.x_{0}^{(l)}\right)$ as $x^{(l)}=\left(1, x_{1}^{(l)}, \ldots, x_{K^{(l)}}^{(l)}\right)^{\prime}$. To initialize the network, similarly define the input layer using the raw predictors, $x^{(0)}=\left(1, z_{1}, \ldots, z_{N}\right)^{\prime}$.

\footnotetext{
${ }^{20}$ Eldan and Shamir (2016) formally demonstrate that depth - even if increased by one layer - can be exponentially more valuable than increasing width in standard feed-forward neural networks. Ever since the seminal work by Hinton et al. (2006), the machine learning community has experimented and adopted deeper (and wider) networks, with as many as 152 layers for image recognition, e.g., He et al. (2016a).

${ }^{21}$ We confine the choices of architectures to a small set of five based on our limited sample size (compared to typical neural network applications).

${ }^{22}$ See, e.g., Jarrett et al. (2009), Nair and Hinton (2010), and Glorot et al. (2011).
} 
The recursive output formula for the neural network at each neuron in layer $l>0$ is then

$$
x_{k}^{(l)}=\operatorname{ReLU}\left(x^{(l-1)^{\prime}} \theta_{k}^{(l-1)}\right),
$$

with final output

$$
g(z ; \theta)=x^{(L-1)^{\prime}} \theta^{(L-1)} .
$$

The number of weight parameters in each hidden layer $l$ is $K^{(l)}\left(1+K^{(l-1)}\right)$, plus another $1+K^{(L-1)}$ weights for the output layer.

Objective Function and Computational Algorithm. We estimate the neural network weight parameters by minimizing the penalized $l_{2}$ objective function of prediction errors. Unlike tree-based algorithms that require "greedy" optimization, training a neural network, in principle, allows for joint updates of all model parameters at each step of the optimization - a substantial advantage of neural networks over trees. However, the high degree of nonlinearity and nonconvexity in neural networks, together with their rich parameterization, make brute force optimization highly computationally intensive (often to the point of infeasibility). A common solution uses stochastic gradient descent (SGD) to train a neural network. Unlike standard descent that uses the entire training sample to evaluate the gradient at each iteration of the optimization, SGD evaluates the gradient from a small random subset of the data. This approximation sacrifices accuracy for enormous acceleration of the optimization routine.

For the same reasons described above (severe nonlinearity and heavy parameterization), regularization of neural networks requires more care than the methods discussed above. In addition to $l_{1}$ penalization of the weight parameters, we simultaneously employ four other regularization techniques in our estimation: learning rate shrinkage, early stopping, batch normalization, and ensembles.

A critical tuning parameter in SGD is the learning rate, which controls the step size of the descent. It is necessary to shrink the learning rate toward zero as the gradient approaches zero, otherwise noise in the calculation of the gradient begins to dominate its directional signal. We adopt the "learning rate shrinkage" algorithm of Kingma and Ba (2014) to adaptively control the learning rate (described further in Algorithm 5 of the Appendix B.3). ${ }^{23}$

Next, "early stopping" is a general machine learning regularization tool. It begins from an initial parameter guess that imposes parsimonious parameterization (for example, setting all $\theta$ values close to zero). In each step of the optimization algorithm, the parameter guesses are gradually updated to reduce prediction errors in the training sample. At each new guess, predictions are also constructed for the validation sample, and the optimization is terminated when the validation sample errors begin to increase. This typically occurs before the prediction errors are minimized in the training sample, hence the name early stopping (see Algorithm 6). By ending the parameter search early, parameters are shrunken toward the initial guess. It is a popular substitute to $l_{2}$ penalization of $\theta$ parameters

\footnotetext{
${ }^{23}$ Relatedly, random subsetting at each SGD iteration adds noise to the optimization procedure, which itself serves as a form of regularization. See, Wilson and Martinez (2003).
} 
because it achieves regularization at a much lower computational cost. ${ }^{24}$ Early stopping can be used alone, or together with $l_{1}$-regularization as we do in this paper.

"Batch normalization" (Ioffe and Szegedy, 2015) is a simple technique for controlling the variability of predictors across different regions of the network and across different datasets. It is motivated by the phenomenon of internal covariate shift in which inputs of hidden layers follow different distributions than their counterparts in the validation sample. This issue is constantly encountered when fitting deep neural networks that involve many parameters and rather complex structures. For each hidden unit in each training step (a "batch"), the algorithm cross-sectionally de-means and variance standardizes the batch inputs to restore the representation power of the unit.

Finally, we adopt an ensemble approach in training our neural networks (see also Hansen and Salamon, 1990; Dietterich, 2000). In particular, we use multiple random seeds to initialize neural network estimation and construct predictions by averaging forecasts from all networks. This reduces prediction variance because the stochastic nature of the optimization can cause different seeds to produce different forecasts. ${ }^{25}$

\subsection{Performance Evaluation}

To assess predictive performance for individual excess stock return forecasts, we calculate the outof-sample $R^{2}$ as

$$
R_{\mathrm{oos}}^{2}=1-\frac{\sum_{(i, t) \in \mathcal{T}_{3}}\left(r_{i, t+1}-\widehat{r}_{i, t+1}\right)^{2}}{\sum_{(i, t) \in \mathcal{T}_{3}} r_{i, t+1}^{2}},
$$

where $\mathcal{T}_{3}$ indicates that fits are only assessed on the testing subsample, whose data never enter into model estimation or tuning. $R_{\text {oos }}^{2}$ pools prediction errors across firms and over time into a grand panel-level assessment of each model.

A subtle but important aspect of our $R^{2}$ metric is that the denominator is the sum of squared excess returns without demeaning. In many out-of-sample forecasting applications, predictions are compared against historical mean returns. While this approach is sensible for the aggregate index or long-short portfolios, for example, it is flawed when it comes to analyzing individual stock returns. Predicting future excess stock returns with historical averages typically underperforms a naive forecast of zero by a large margin. That is, the historical mean stock return is so noisy that it artificially lowers the bar for "good" forecasting performance. We avoid this pitfall by benchmarking our $R^{2}$ against a forecast value of zero. To give an indication of the importance of this choice, when we benchmark model predictions against historical mean stock returns, the out-of-sample monthly $R^{2}$ of all methods rises by roughly three percentage points.

\footnotetext{
${ }^{24}$ Early stopping bears a comparatively low computation cost because it only partially optimizes, while the $l_{2}$ regularization, or more generally elastic net, search across tuning parameters and fully optimizes the model subject to each tuning parameter guess. As usual, elastic net's $l_{1}$-penalty component encourages neurons to connect to limited number of other neurons, while its $l_{2}$-penalty component shrinks the weight parameters toward zero (a feature known in the neural net literature as "weight-decay"). In certain circumstances, early stopping and weight-decay are shown to be equivalent. See, e.g., Bishop (1995) and Goodfellow et al. (2016).

${ }^{25}$ Estimation with different seeds can run independently in parallel which limits incremental computing time.
} 
To make pairwise comparisons of methods, we use the Diebold and Mariano (1995) test for differences in out-of-sample predictive accuracy between two models. ${ }^{26}$ While time series dependence in returns is sufficiently weak, it is unlikely that the conditions of weak error dependence underlying the Diebold-Mariano test apply to our stock-level analysis due of potentially strong dependence in the cross section. We adapt Diebold-Mariano to our setting by comparing the cross-sectional average of prediction errors from each model, instead of comparing errors among individual returns. More precisely, to test the forecast performance of method (1) versus (2), we define the test statistic $D M_{12}=\bar{d}_{12} / \widehat{\sigma}_{\bar{d}_{12}}$, where

$$
d_{12, t+1}=\frac{1}{n_{3, t+1}} \sum_{i=1}^{n_{3}}\left(\left(\widehat{e}_{i, t+1}^{(1)}\right)^{2}-\left(\widehat{e}_{i, t+1}^{(2)}\right)^{2}\right),
$$

$\widehat{e}_{i, t+1}^{(1)}$ and $\widehat{e}_{i, t+1}^{(2)}$ denote the prediction error for stock return $i$ at time $t$ using each method, and $n_{3, t+1}$ is the number of stocks in the testing sample (year $\left.t+1\right)$. Then $\bar{d}_{12}$ and $\widehat{\sigma}_{\bar{d}_{12}}$ denote the mean and Newey-West standard error of $d_{12, t}$ over the testing sample. This modified DieboldMariano test statistic, which is now based on a single time series $d_{12, t+1}$ of error differences with little autocorrelation, is more likely to satisfy the mild regularity conditions needed for asymptotic normality and in turn provide appropriate $p$-values for our model comparison tests.

\subsection{Variable Importance and Marginal Relationships}

Our goal in interpreting machine learning models is modest. We aim to identify covariates that have an important influence on the cross-section of expected returns while simultaneously controlling for the many other predictors in the system.

We discover influential covariates by ranking them according to a notion of variable importance, which we denote as $\mathrm{VI}_{j}$ for the $j^{\text {th }}$ input variable. We consider two different notions of importance. The first is the reduction in panel predictive $R^{2}$ from setting all values of predictor $j$ to zero, while holding the remaining model estimates fixed (used, for example, in the context of dimension reduction by Kelly et al., 2019). The second, proposed in the neural networks literature by Dimopoulos et al. (1995), is the sum of squared partial derivatives (SSD) of the model to each input variable $j$, which summarizes the sensitivity of model fits to changes in that variable. ${ }^{27}$

As part of our analysis, we also trace out the marginal relationship between expected returns

\footnotetext{
${ }^{26}$ As emphasize by Diebold (2015), the model-free nature of the Diebold-Mariano test means that it should be interpreted as a comparison of forecasts, and not as a comparison of "fully articulated econometric models."

${ }^{27}$ In particular, SSD defines the $j^{\text {th }}$ variable importance as$$
S S D_{j}=\sum_{i, t \in \mathcal{T}_{1}}\left(\left.\frac{\partial g(z ; \theta)}{\partial z_{j}}\right|_{z=z_{i, t}}\right)^{2},
$$

where, with a slight abuse of notation, $z_{j}$ in the denominator of the derivative denotes the $j^{\text {th }}$ element of the vector of input variables. We measure SSD within the training set, $\mathcal{T}_{1}$. Note that due to non-differentiabilities in tree-based models, the Dimopoulos et al. (1995) method is not applicable. Therefore, when we conduct this second variable importance analysis, we measure variable importance for random forests and boosted trees using mean decrease in impurity (see, e.g., Friedman, 2001).
} 
and each characteristic. Despite obvious limitations, such a plot is an effective tool for visualizing the first-order impact of covariates in a machine learning model.

\section{An Empirical Study of US Equities}

\subsection{Data and Over-arching Model}

We obtain monthly total individual equity returns from CRSP for all firms listed in the NYSE, AMEX, and NASDAQ. Our sample begins in March 1957 (the start date of the S\&P 500) and ends in December 2016, totaling 60 years. The number of stocks in our sample is almost 30,000, with the average number of stocks per month exceeding $6,200 .{ }^{28}$ We also obtain the Treasury-bill rate to proxy for the risk-free rate from which we calculate individual excess returns.

In addition, we build a large collection of stock-level predictive characteristics based on the cross section of stock returns literature. These include 94 characteristics $^{29}$ (61 of which are updated annually, 13 updated quarterly, and 20 updated monthly). In addition, we include 74 industry dummies corresponding to the first two digits of Standard Industrial Classification (SIC) codes. We provide the details of these characteristics in Table A.6. ${ }^{30}$

We also construct eight macroeconomic predictors following the variable definitions detailed in Welch and Goyal (2008), including dividend-price ratio (dp), earnings-price ratio (ep), book-tomarket ratio (bm), net equity expansion (ntis), Treasury-bill rate (tbl), term spread (tms), default spread (dfy), and stock variance (svar). ${ }^{31}$

All of the machine learning methods we consider are designed to approximate the over-arching empirical model $\mathrm{E}_{t}\left(r_{i, t+1}\right)=g^{\star}\left(z_{i, t}\right)$ defined in equation (2). Throughout our analysis we define the baseline set of stock-level covariates $z_{i, t}$ as

$$
z_{i, t}=x_{t} \otimes c_{i, t}
$$

\footnotetext{
${ }^{28}$ We include stocks with prices below $\$ 5$, share codes beyond 10 and 11 , and financial firms. There are at least three important reasons why we select the largest possible pool of assets. First, these commonly used filters remove certain stocks that are components of the S\&P 500 index, and we find it clearly problematic to exclude such important stocks from an asset pricing analysis. Moreover, because we aggregate individual stock return predictions to predict the index, we cannot omit such stocks. Second, our results are less prone to sample selection or data snooping biases that the literature, e.g. Lo and MacKinlay (1990), cautions against. Third, using a larger sample helps avoid overfitting by increasing the ratio of observation count to parameter count. That said, our results are qualitatively identical and quantitively unchanged if we filter out these firms.

${ }^{29}$ We cross-sectionally rank all stock characteristics period-by-period and map these ranks into the $[-1,1]$ interval following Kelly et al. (2019) and Freyberger et al. (2019).

${ }^{30}$ The 94 predictive characteristics are based on Green et al. (2017), and we adapt the SAS code available from Jeremiah Green's website and extend the sample period back to 1957. Our data construction differs by adhering more closely to variable definitions in original papers. For example, we construct book-equity and operating profitability following Fama and French (2015). Most of these characteristics are released to the public with a delay. To avoid the forward-looking bias, we assume that monthly characteristics are delayed by at most 1 month, quarterly with at least 4 months lag, and annual with at least 6 months lag. Therefore, in order to predict returns at month $t+1$, we use most recent monthly characteristics at the end of month $t$, most recent quarterly data by end $t-4$, and most recent annual data by end $t-6$. Another issue is missing characteristics, which we replace with the cross-sectional median at each month for each stock, respectively.

${ }^{31}$ The monthly data are available from Amit Goyal's website.
} 
where $c_{i, t}$ is a $P_{c} \times 1$ matrix of characteristics for each stock $i$, and $x_{t}$ is a $P_{x} \times 1$ vector of macroeconomic predictors (and are thus common to all stocks, including a constant). Thus, $z_{i, t}$ is a $P \times 1$ vector of features for predicting individual stock returns (with $P=P_{c} P_{x}$ ) and includes interactions between stock-level characteristics and macroeconomic state variables. The total number of covariates is $94 \times(8+1)+74=920$.

The over-arching model specified by (2) and (21) nests many models proposed in the literature (Rosenberg, 1974; Harvey and Ferson, 1999, among others). The motivating example for this model structure is the standard beta-pricing representation of the asset pricing conditional Euler equation,

$$
\mathrm{E}_{t}\left(r_{i, t+1}\right)=\beta_{i, t}^{\prime} \lambda_{t}
$$

The structure of our feature set in (21) allows for purely stock-level information to enter expected returns via $c_{i, t}$ in analogy with the risk exposure function $\beta_{i, t}$, and also allows aggregate economic conditions to enter in analogy with the dynamic risk premium $\lambda_{t}$. In particular, if $\beta_{i, t}=\theta_{1} c_{i, t}$, and $\lambda_{t}=\theta_{2} x_{t}$, for some constant parameter matrices $\theta_{1}\left(K \times P_{c}\right)$ and $\theta_{2}\left(K \times P_{x}\right)$, then the beta-pricing model in (22) becomes

$$
g^{\star}\left(z_{i, t}\right)=\mathrm{E}_{t}\left(r_{i, t+1}\right)=\beta_{i, t}^{\prime} \lambda_{t}=c_{i, t}^{\prime} \theta_{1}^{\prime} \theta_{2} x_{t}=\left(x_{t} \otimes c_{i, t}\right)^{\prime} \operatorname{vec}\left(\theta_{1}^{\prime} \theta_{2}\right)=: z_{i, t}^{\prime} \theta,
$$

where $\theta=\operatorname{vec}\left(\theta_{1}^{\prime} \theta_{2}\right)$. The over-arching model is more general than this example because $g^{\star}(\cdot)$ is not restricted to be a linear function. Considering nonlinear $g^{\star}(\cdot)$ formulations, for example via generalized linear models or neural networks, essentially expands the feature set to include a variety of functional transformations of the baseline $z_{i, t}$ predictor set.

We divide the 60 years of data into 18 years of training sample (1957 - 1974), 12 years of validation sample (1975 - 1986), and the remaining 30 years (1987 - 2016) for out-of-sample testing. Because machine learning algorithms are computationally intensive, we avoid recursively refitting models each month. Instead, we refit once every year as most of our signals are updated once per year. Each time we refit, we increase the training sample by one year. We maintain the same size of the validation sample, but roll it forward to include the most recent twelve months. ${ }^{32}$

\subsection{The Cross Section of Individual Stocks}

Table 1 presents the comparison of machine learning techniques in terms of their out-of-sample predictive $R^{2}$. We compare thirteen models in total, including OLS with all covariates, OLS-3 (which pre-selects size, book-to-market, and momentum as the only covariates), PLS, PCR, elastic net (ENet), generalized linear model with group lasso (GLM), random forest (RF), gradient boosted regression trees (GBRT), and neural network architectures with one to five layers (NN1,..,NN5). For OLS, ENet, GLM, and GBRT, we present their robust versions using Huber loss, which perform better than the version without.

The first row of Table 1 reports $R_{\text {oos }}^{2}$ for the entire pooled sample. The OLS model using all 920

\footnotetext{
${ }^{32}$ Note that we do not use cross-validation in order to maintain the temporal ordering of the data.
} 
Table 1: Monthly Out-of-sample Stock-level Prediction Performance (Percentage $R_{\text {oos }}^{2}$ )

\begin{tabular}{|c|c|c|c|c|c|c|c|c|c|c|c|c|c|}
\hline & $\begin{array}{c}\text { OLS } \\
+\mathrm{H}\end{array}$ & $\begin{array}{c}\text { OLS-3 } \\
+\mathrm{H}\end{array}$ & PLS & PCR & $\begin{array}{c}\text { ENet } \\
+\mathrm{H}\end{array}$ & $\begin{array}{c}\text { GLM } \\
+\mathrm{H}\end{array}$ & $\mathrm{RF}$ & $\begin{array}{c}\text { GBRT } \\
+\mathrm{H}\end{array}$ & NN1 & NN2 & NN3 & NN4 & NN5 \\
\hline All & -3.46 & 0.16 & 0.27 & 0.26 & 0.11 & 0.19 & 0.33 & 0.34 & 0.33 & 0.39 & 0.40 & 0.39 & 0.36 \\
\hline Top 1000 & -11.28 & 0.31 & -0.14 & 0.06 & 0.25 & 0.14 & 0.63 & 0.52 & 0.49 & 0.62 & 0.70 & 0.67 & 0.64 \\
\hline Bottom 1000 & -1.30 & 0.17 & 0.42 & 0.34 & 0.20 & 0.30 & 0.35 & 0.32 & 0.38 & 0.46 & 0.45 & 0.47 & 0.42 \\
\hline
\end{tabular}

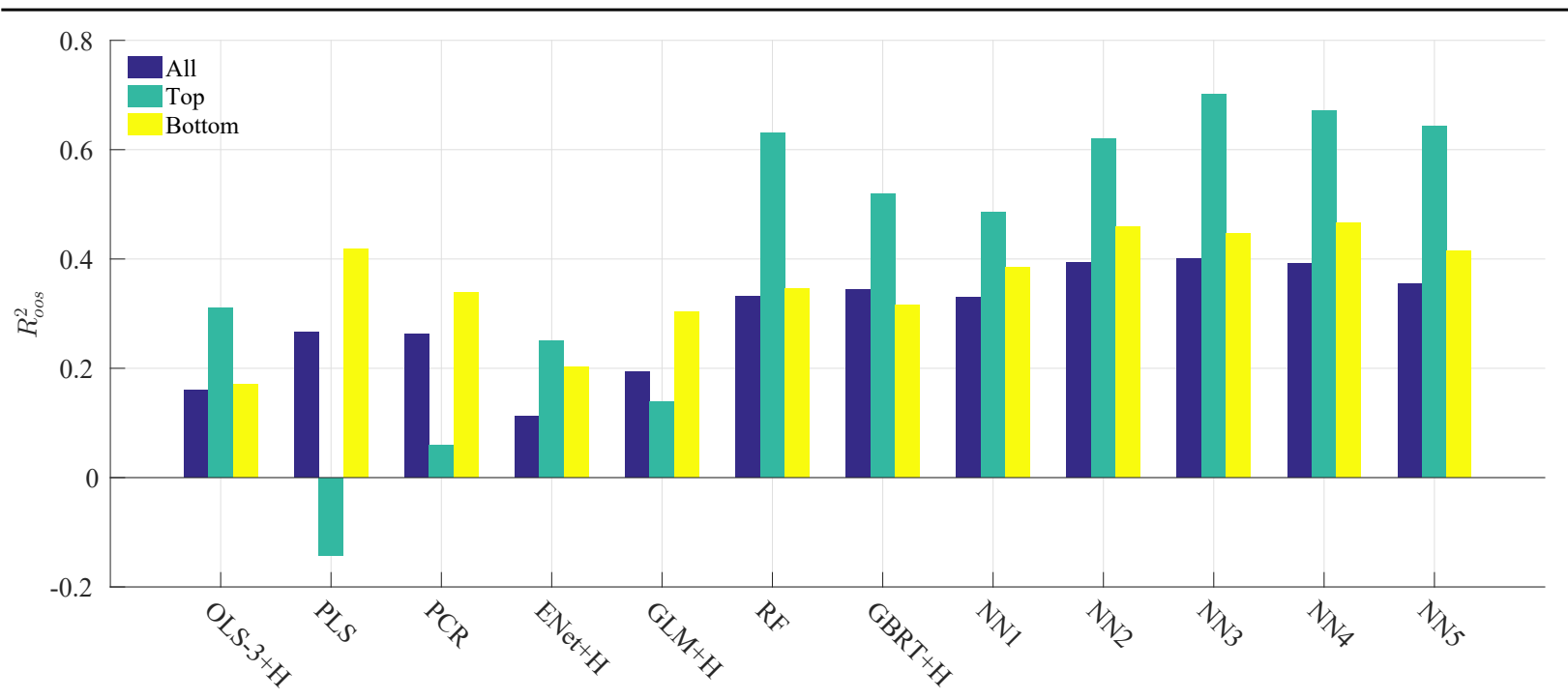

Note: In this table, we report monthly $R_{\text {oos }}^{2}$ for the entire panel of stocks using OLS with all variables (OLS), OLS using only size, book-to-market, and momentum (OLS-3), PLS, PCR, elastic net (ENet), generalize linear model (GLM), random forest (RF), gradient boosted regression trees (GBRT), and neural networks with one to five layers (NN1-NN5). " $+\mathrm{H}$ " indicates the use of Huber loss instead of the $l_{2}$ loss. We also report these $R_{\text {oos }}^{2}$ within subsamples that include only the top 1,000 stocks or bottom 1,000 stocks by market value. The lower panel provides a visual comparison of the $R_{\text {oos }}^{2}$ statistics in the table (omitting OLS due to its large negative values).

features produces an $R_{\text {oos }}^{2}$ of $-3.46 \%$, indicating it is handily dominated by applying a naive forecast of zero to all stocks in all months. This may be unsurprising as the lack of regularization leaves OLS highly susceptible to in-sample overfit. However, restricting OLS to a sparse parameterization, either by forcing the model to include only three covariates (size, value, and momentum), or by penalizing the specification with the elastic net - generates a substantial improvement over the full OLS model $\left(R_{\mathrm{oos}}^{2}\right.$ of $0.16 \%$ and $0.11 \%$ respectively). Figure 3 summarizes the complexity of each model at each re-estimation date. The upper left panel shows the number of features to which elastic net assigns a non-zero loading. In the first ten years of the test sample, the model typically chooses fewer than five features. After 2000, the number of selected features rises and hovers between 20 and 40 .

Regularizing the linear model via dimension reduction improves predictions even further. By forming a few linear combinations of predictors, PLS and especially PCR, raise the out-of-sample $R^{2}$ to $0.27 \%$ and $0.26 \%$, respectively. Figure 3 shows that PCR typically uses 20 to 40 components in its forecasts. PLS, on the other hand, fails to find a single reliable component for much of the early sample, but eventually settles on three to six components. The improvement of dimension 
Figure 3: Time-varying Model Complexity
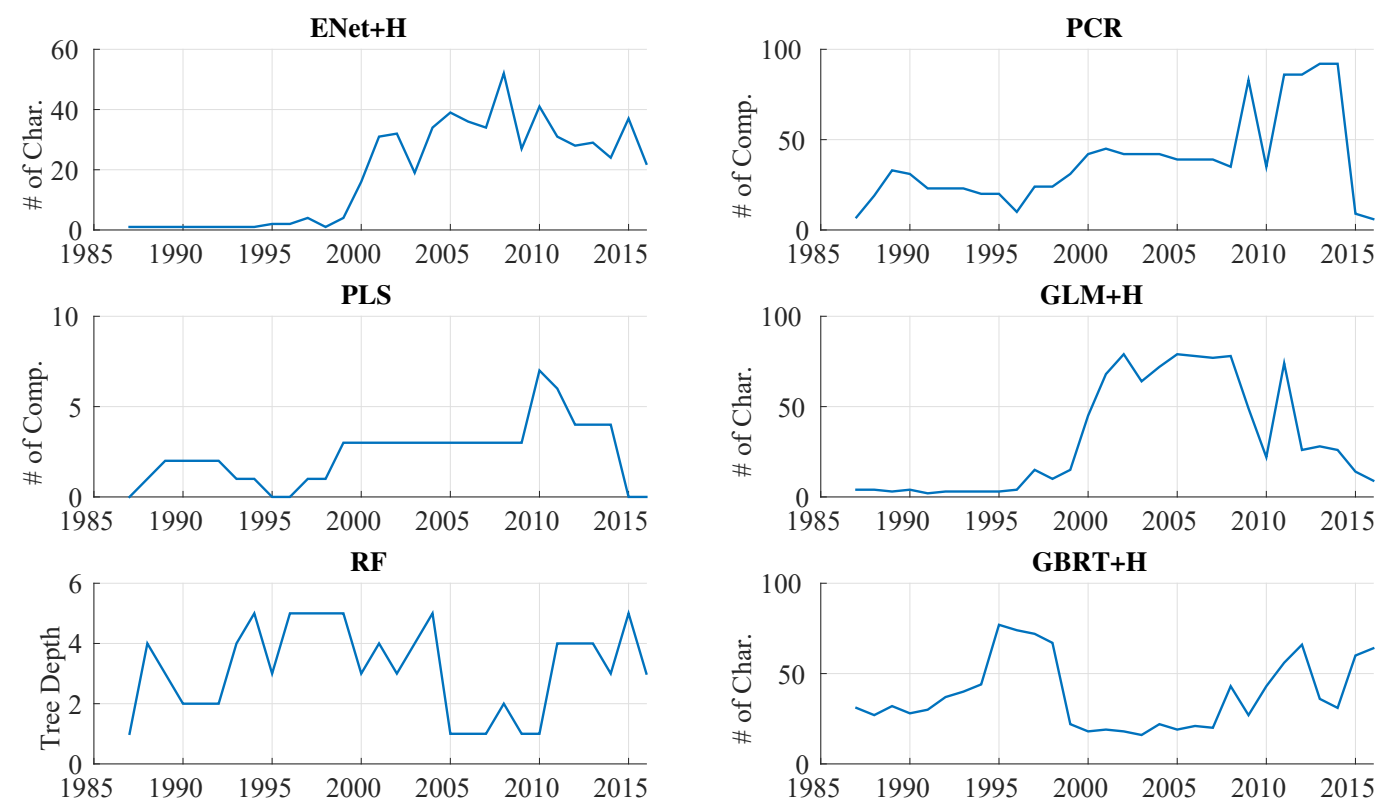

Note: This figure demonstrates the model complexity for elastic net (ENet), PCR, PLS, generalized linear model with group lasso (GLM), random forest (RF) and gradient boosted regression trees (GBRT) in each training sample of our 30-year recursive out-of-sample analysis. For ENet and GLM we report the number of features selected to have non-zero coefficients; for PCR and PLS we report the number of selected components; for RF we report the average tree depth; and for GBRT we report the number of distinct characteristics entering into the trees.

reduction over variable selection via elastic net suggests that characteristics are partially redundant and fundamentally noisy signals. Combining them into low-dimension components averages out noise to better reveal their correlated signals.

The generalized linear model with group lasso penalty fails to improve on the performance of purely linear methods $\left(R_{\text {oos }}^{2}\right.$ of $\left.0.19 \%\right)$. The fact that this method uses spline functions of individual features, but includes no interaction among features, suggests that univariate expansions provide little incremental information beyond the linear model. Though it tends to select more features than elastic net, those additional features do not translate into incremental performance.

Boosted trees and random forests are competitive with PCR, producing fits of $0.34 \%$ and $0.33 \%$, respectively. Random forests generally estimate shallow trees, with one to five layers on average. To quantify the complexity of GBRT, we report the number of features used in the boosted tree ensemble at each re-estimation point. In the beginning of the sample GBRT uses around 30 features to partition outcomes, with this number increasing to 50 later in the sample.

Neural networks are the best performing nonlinear method, and the best predictor overall. The $R_{\text {oos }}^{2}$ is $0.33 \%$ for NN1 and peaks at $0.40 \%$ for NN3. These results point to the value of incorporating complex predictor interactions, which are embedded in tree and neural network models but that are 
Table 2: Annual Out-of-sample Stock-level Prediction Performance (Percentage $R_{\mathrm{oos}}^{2}$ )

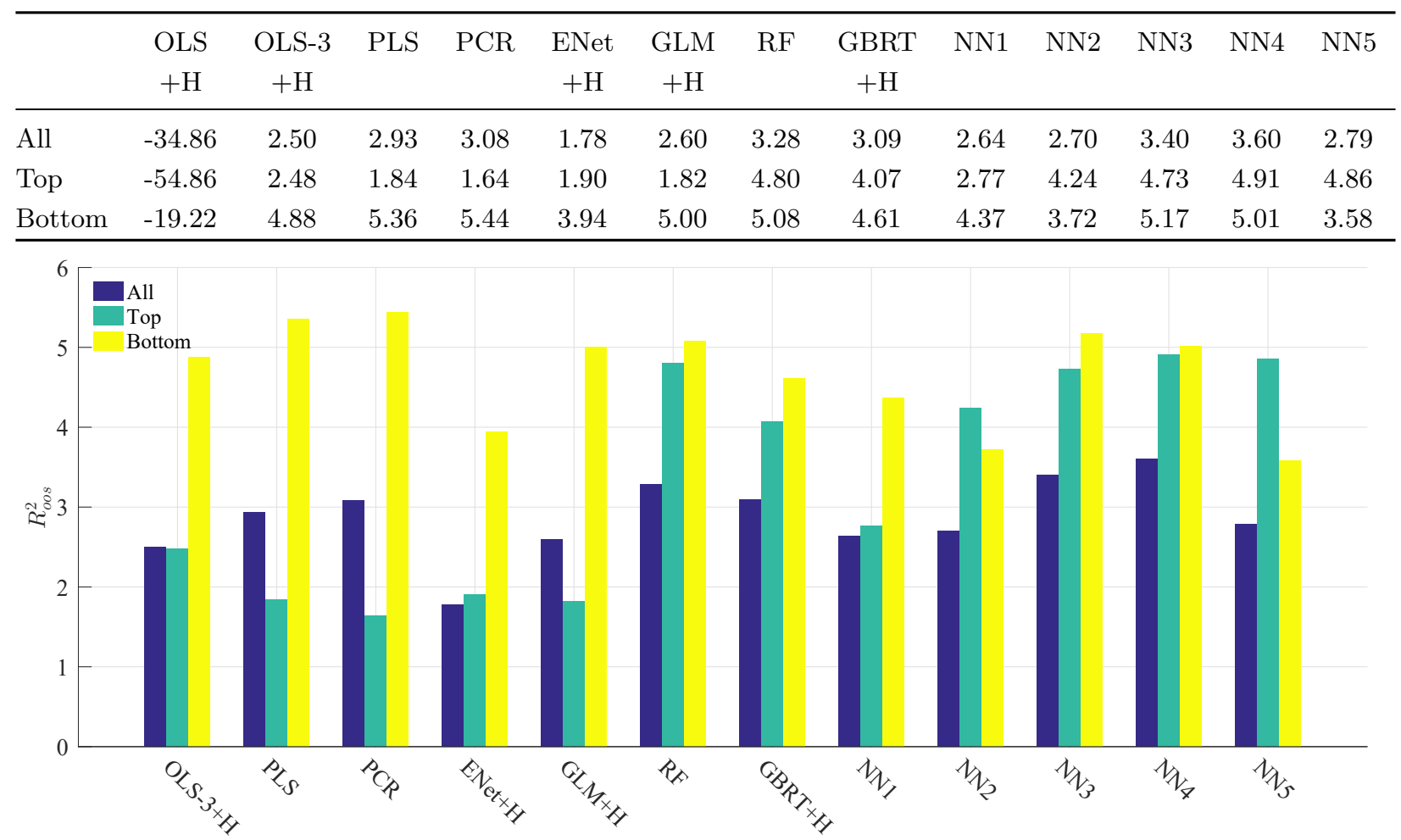

Note: Annual return forecasting $R_{\text {oos }}^{2}$ (see Table 1 notes).

missed by other techniques. The results also show that in the monthly return setting, the benefits of "deep" learning are limited, as four and five layer models fail to improve over NN3. ${ }^{33}$

The second and third rows of Table 1 break out predictability for large stocks (the top 1,000 stocks by market equity each month) and small stocks (the bottom 1,000 each month). This is based on the full estimated model (using all stocks), but focuses on fits among the two subsamples. The baseline patterns that OLS fares poorly, regularized linear models are an improvement, and nonlinear models dominate carries over into subsamples. Tree methods and neural networks are especially successful among large stocks, with $R_{\text {oos }}^{2}$ ranging from $0.52 \%$ to $0.70 \%$. This dichotomy provides reassurance that machine learning is not merely picking up small scale inefficiencies driven by illiquidity. ${ }^{34}$

Table 2 conducts our analysis at the annual horizon. The comparative performance across different methods is similar to the monthly results shown in Table 1 , but the annual $R_{\text {oos }}^{2}$ is nearly an order of magnitude larger. Their success in forecasting annual returns likewise illustrates that

\footnotetext{
${ }^{33}$ Because we hold the five neural networks architectures fixed and simply compare across them, we do not describe their estimated complexity in Figure 3.

${ }^{34} \mathrm{As}$ an aside, it is useful to know that there is a roughly $3 \%$ inflation in out-of-sample $R^{2} \mathrm{~s}$ if performance is benchmarked against historical averages. For OLS-3, the $R^{2}$ relative to the historical mean forecast is $3.74 \%$ per month! Evidently, the historical mean is such a noisy forecaster that it is easily beaten by a fixed excess return forecasts of zero.
} 
Table 3: Comparison of Monthly Out-of-Sample Prediction using Diebold-Mariano Tests

\begin{tabular}{|c|c|c|c|c|c|c|c|c|c|c|c|c|}
\hline & $\begin{array}{c}\text { OLS-3 } \\
+\mathrm{H}\end{array}$ & PLS & PCR & $\begin{array}{c}\text { ENet } \\
+\mathrm{H}\end{array}$ & $\begin{array}{c}\text { GLM } \\
+\mathrm{H}\end{array}$ & $\mathrm{RF}$ & $\begin{array}{c}\text { GBRT } \\
+\mathrm{H}\end{array}$ & NN1 & NN2 & NN3 & NN4 & NN5 \\
\hline$\overline{\mathrm{OLS}}+\mathrm{H}$ & $3.26^{*}$ & $3.29^{*}$ & $3.35^{*}$ & $3.29^{*}$ & $3.28^{*}$ & $3.29^{*}$ & $3.26^{*}$ & $3.34^{*}$ & $3.40^{*}$ & $3.38^{*}$ & $3.37^{*}$ & $3.38^{*}$ \\
\hline OLS-3+H & & 1.42 & 1.87 & -0.27 & 0.62 & 1.64 & 1.28 & 1.25 & 2.13 & 2.13 & 2.36 & 2.11 \\
\hline PLS & & & -0.19 & -1.18 & -1.47 & 0.87 & 0.67 & 0.63 & 1.32 & 1.37 & 1.66 & 1.08 \\
\hline PCR & & & & -1.10 & -1.37 & 0.85 & 0.75 & 0.58 & 1.17 & 1.19 & 1.34 & 1.00 \\
\hline $\mathrm{ENet}+\mathrm{H}$ & & & & & 0.64 & 1.90 & 1.40 & 1.73 & 1.97 & 2.07 & 1.98 & 1.85 \\
\hline $\mathrm{GLM}+\mathrm{H}$ & & & & & & 1.76 & 1.22 & 1.29 & 2.28 & 2.17 & $2.68^{*}$ & 2.37 \\
\hline $\mathrm{RF}$ & & & & & & & 0.07 & -0.03 & 0.31 & 0.37 & 0.34 & 0.00 \\
\hline $\mathrm{GBRT}+\mathrm{H}$ & & & & & & & & -0.06 & 0.16 & 0.21 & 0.17 & -0.04 \\
\hline NN1 & & & & & & & & & 0.56 & 0.59 & 0.45 & 0.04 \\
\hline NN2 & & & & & & & & & & 0.32 & -0.03 & -0.88 \\
\hline NN3 & & & & & & & & & & & -0.32 & -0.92 \\
\hline NN4 & & & & & & & & & & & & -1.04 \\
\hline
\end{tabular}

Note: This table reports pairwise Diebold-Mariano test statistics comparing the out-of-sample stock-level prediction performance among thirteen models. Positive numbers indicate the column model outperforms the row model. Bold font indicates the difference is significant at $5 \%$ level or better for individual tests, while an asterisk indicates significance at the $5 \%$ level for 12-way comparisons via our conservative Bonferroni adjustment.

machine learning models are able to isolate risk premia that persist over business cycle frequencies and are not merely capturing short-lived inefficiencies.

While Table 1 offers a quantitative comparison of models' predictive performance, Table 3 assesses the statistical significance of differences among models at the monthly frequency. It reports DieboldMariano test statistics for pairwise comparisons of a column model versus a row model. DieboldMariano statistics are distributed $\mathcal{N}(0,1)$ under the null of no difference between models, thus the test statistic magnitudes map to $p$-values in the same way as regression $t$-statistics. Our sign convention is that a positive statistic indicates the column model outperforms the row model. Bold numbers denote significance at the $5 \%$ level for each individual test.

The first conclusion from Table 3 is that constrained linear models - including restricting OLS to only use three predictors, reducing dimension via PLS or PCA, and penalizing via elastic netproduce statistically significant improvements over the unconstrained OLS model. Second, we see little difference in the performance of penalized linear methods and dimension reduction methods. Third, we find that tree-based methods uniformly improve over linear models, but the improvements are at best marginally significant. Neural networks are the only models that produce large and significant statistical improvements over linear and generalized linear models. They also improve over tree models, but the difference is not statistically significant.

Table 3 makes multiple comparisons. We highlight how inference changes under a conservative Bonferroni multiple comparisons correction that divides the significance level by the number of comparisons. ${ }^{35}$ For a significance level of $5 \%$ amid 12 model comparisons, the adjusted one-sided

\footnotetext{
${ }^{35}$ Multiple comparisons are a concern when the researcher conducts many hypotheses tests and draws conclusions based on only those that are significant. This distorts the size of tests through a selection bias. This is not how we present our results - we report $t$-statistics for every comparison we consider-yet we report adjusted inference to err on the side of caution. We also note that false discoveries in multiple comparisons should be randomly distributed.
} 
critical value in our setting is 2.64. In the table, tests that exceed this conservative threshold are accompanied by an asterisk. The main difference with a Bonferroni adjustment is that neural networks become only marginally significant over penalized linear models.

\subsection{Which Covariates Matter?}

We now investigate the relative importance of individual covariates for the performance of each model using the importance measures described in Section 2.9. To begin, for each method, we calculate the reduction in $R^{2}$ from setting all values of a given predictor to zero within each training sample, and average these into a single importance measure for each predictor. Figure 4 reports the resulting importances of the top 20 stock-level characteristics for each method. Variable importances within a model are normalized to sum to one, giving them the interpretation of relative importance for that particular model.

Figure 5 reports overall rankings of characteristics for all models. We rank the importance of each characteristic for each method, then sum their ranks. Characteristics are ordered so that the highest total ranks are on top and the lowest ranking characteristics are at the bottom. The color gradient within each column shows the model-specific ranking of characteristics from least to most important (lightest to darkest). ${ }^{36}$

Figures 4 and 5 demonstrate that models are generally in close agreement regarding the most influential stock-level predictors, which can be grouped into four categories. The first are based on recent price trends, including five of the top seven variables in Figure 5: short-term reversal (mom1m), stock momentum (mom12m), momentum change (chmom), industry momentum (indmom), recent maximum return (maxret), and long-term reversal (mom36m). Next are liquidity variables, including turnover and turnover volatility (turn, std_turn), log market equity (mvel1), dollar volume (dolvol), Amihud illiquidity (ill), number of zero trading days (zerotrade), and bidask spread (baspread). Risk measures constitute the third influential group, including total and idiosyncratic return volatility (retvol, idiovol), market beta (beta), and beta-squared (betasq). The last group includes valuation ratios and fundamental signals, such as earnings-to-price (ep), sales-toprice (sp), asset growth (agr), and number of recent earnings increases (nincr). Figure 4 shows that characteristic importance magnitudes for penalized linear models and dimension reduction models are highly skewed toward momentum and reversal. Trees and neural networks are more democratic, drawing predictive information from a broader set of characteristics.

We find that our second measure of variable importance, SSD from Dimopoulos et al. (1995), produces very similar results to the simpler $R^{2}$ measure. Within each model, we calculate the Pearson correlation between relative importances from SSD and the $R^{2}$ measure. These correlations range from $84.0 \%$ on the low end (NN1) to $97.7 \%$ on the high end (random forest). That is, the two

\footnotetext{
The statistically significant $t$-statistics in our analyses do not appear random, but instead follow a pattern in which nonlinear models outperform linear ones.

${ }^{36}$ Figure 5 is based on the average rank over 30 recursing training samples. Figure A.1 presents the ranks for each of the recursing sample, respectively. The rank of important characteristics (top third of the covariates) are remarkably stable over time. This is true for all models, though we show results for one representative model (NN3) in the interest of space.
} 
Figure 4: Variable Importance By Model

PLS
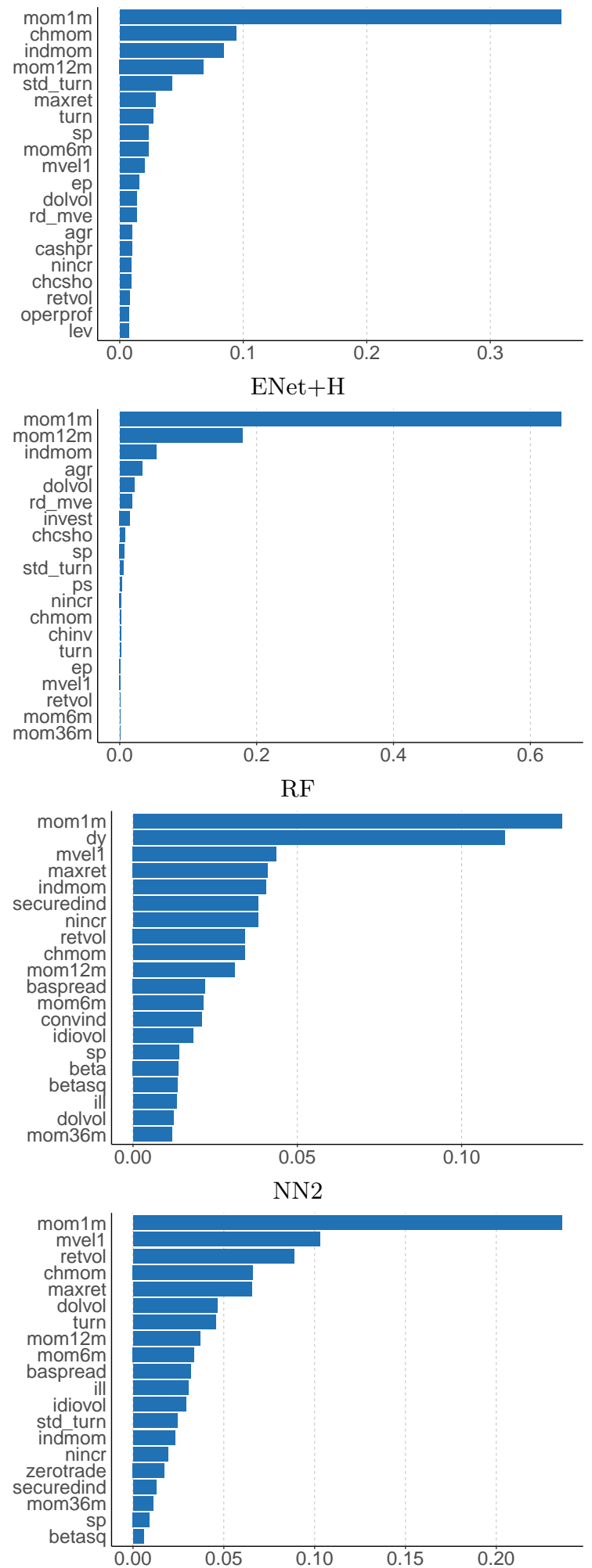

PCR

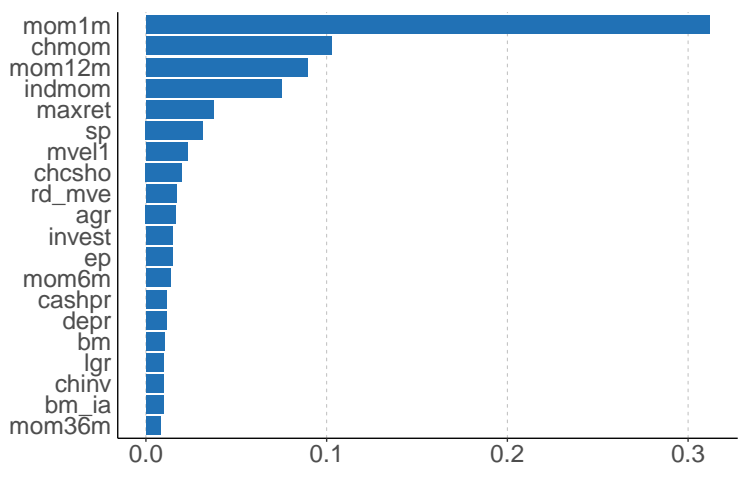

$\mathrm{GLM}+\mathrm{H}$

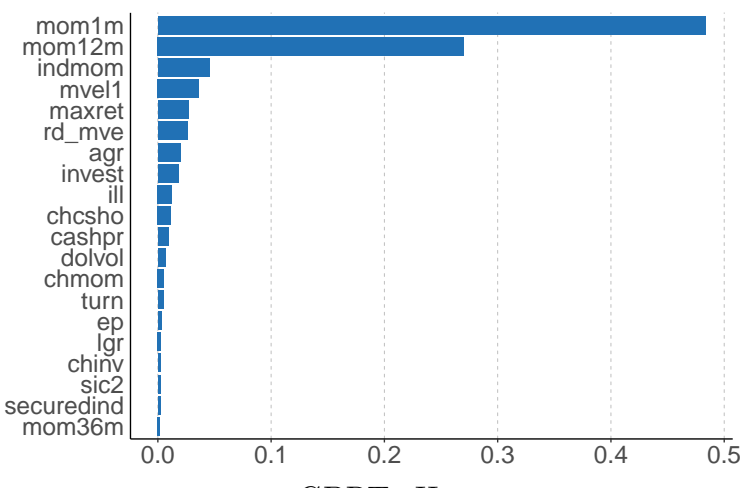

$\mathrm{GBRT}+\mathrm{H}$
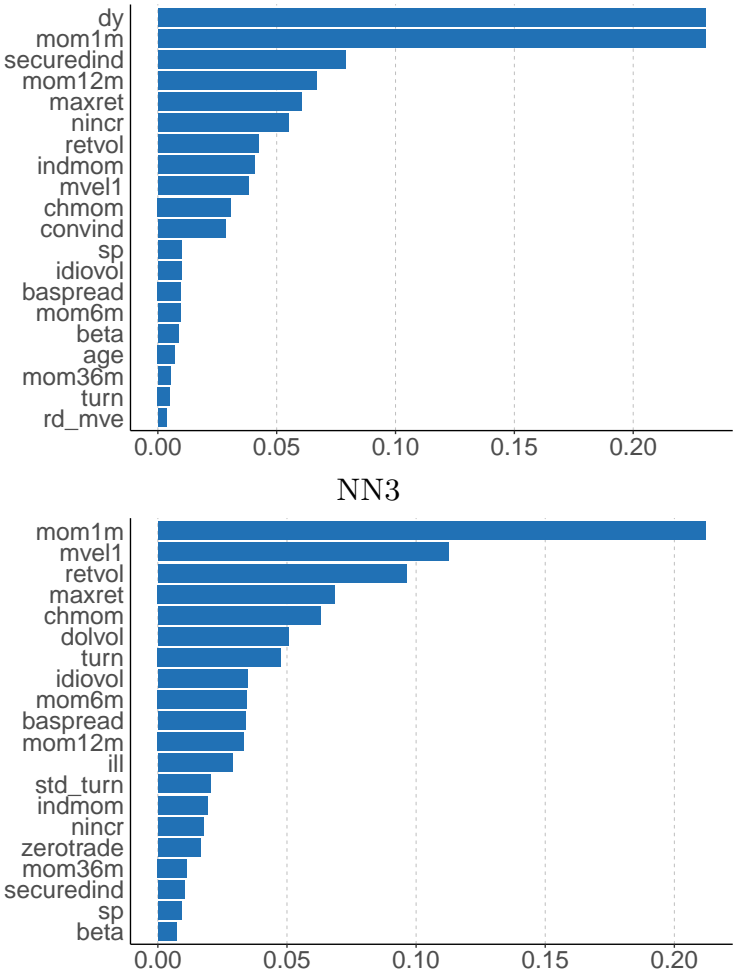

Note: Variable importance for the top 20 most influential variables in each model. Variable importance is an average over all training samples. Variable importances within each model are normalized to sum to one. 
Figure 5: Characteristic Importance

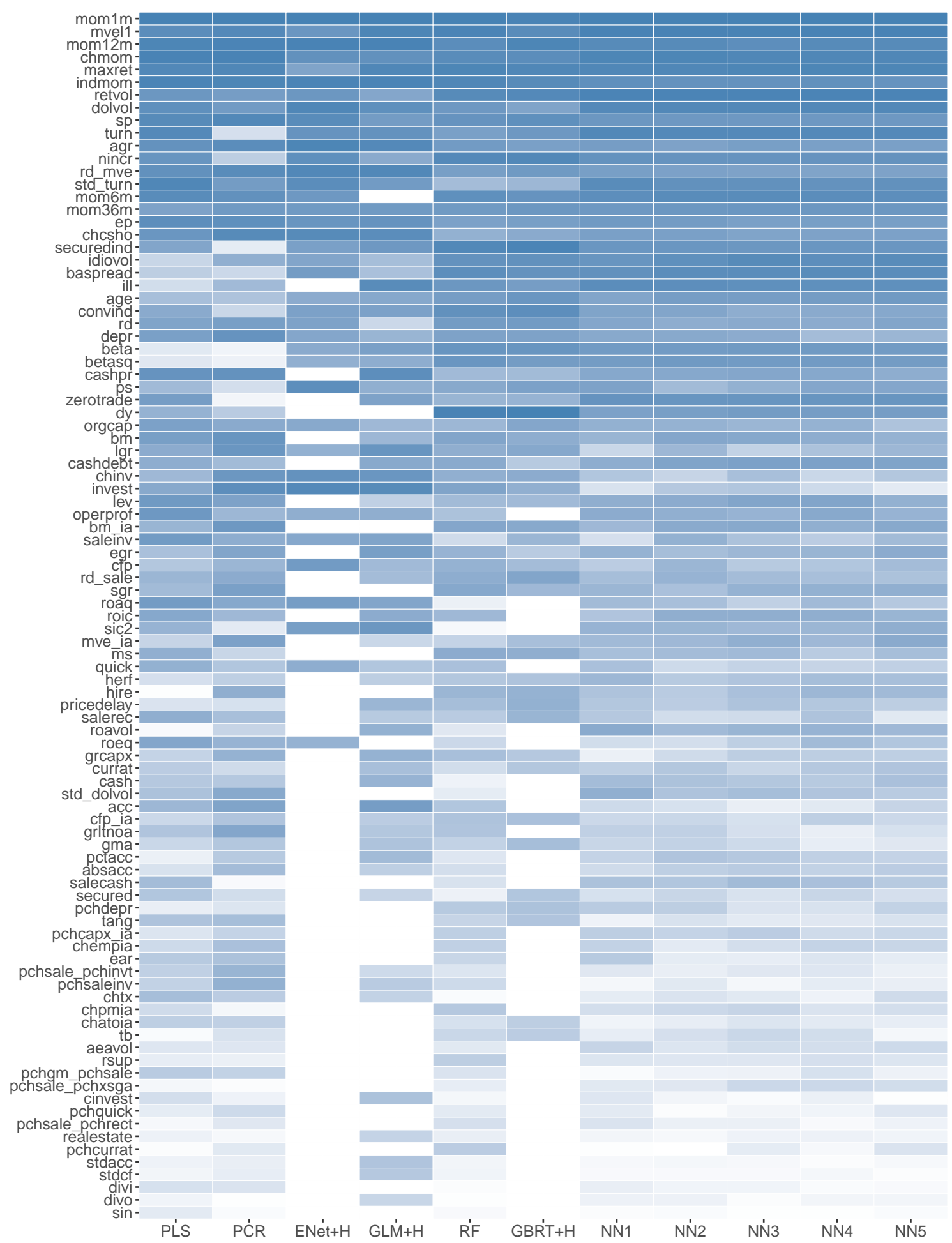

Note: Rankings of 94 stock-level characteristics and the industry dummy (sic2) in terms of overall model contribution. Characteristics are ordered based on the sum of their ranks over all models, with the most influential characteristics on top and least influential on bottom. Columns correspond to individual models, and color gradients within each column indicate the most influential (dark blue) to least influential (white) variables. 
Table 4: Variable Importance for Macroeconomic Predictors

\begin{tabular}{lrrrrrrrrrrr}
\hline & PLS & PCR & ENet+H & GLM+H & RF & GBRT+H & NN1 & NN2 & NN3 & NN4 & NN5 \\
\hline dp & 12.52 & 14.12 & 2.49 & 4.54 & 5.80 & 6.05 & 15.57 & 17.58 & 14.84 & 13.95 & 13.15 \\
ep & 12.25 & 13.52 & 3.27 & 7.37 & 6.27 & 2.85 & 8.86 & 8.09 & 7.34 & 6.54 & 6.47 \\
bm & 14.21 & 14.83 & 33.95 & 43.46 & 10.94 & 12.49 & 28.57 & 27.18 & 27.92 & 26.95 & 27.90 \\
ntis & 11.25 & 9.10 & 1.30 & 4.89 & 13.02 & 13.79 & 18.37 & 19.26 & 20.15 & 19.59 & 18.68 \\
tbl & 14.02 & 15.29 & 13.29 & 7.90 & 11.98 & 19.49 & 17.18 & 16.40 & 17.76 & 20.99 & 21.06 \\
tms & 11.35 & 10.66 & 0.31 & 5.87 & 16.81 & 15.27 & 10.79 & 10.59 & 10.91 & 10.38 & 10.33 \\
dfy & 17.17 & 15.68 & 42.13 & 24.10 & 24.37 & 22.93 & 0.09 & 0.06 & 0.06 & 0.04 & 0.12 \\
svar & 7.22 & 6.80 & 3.26 & 1.87 & 10.82 & 7.13 & 0.57 & 0.85 & 1.02 & 1.57 & 2.29 \\
\hline
\end{tabular}

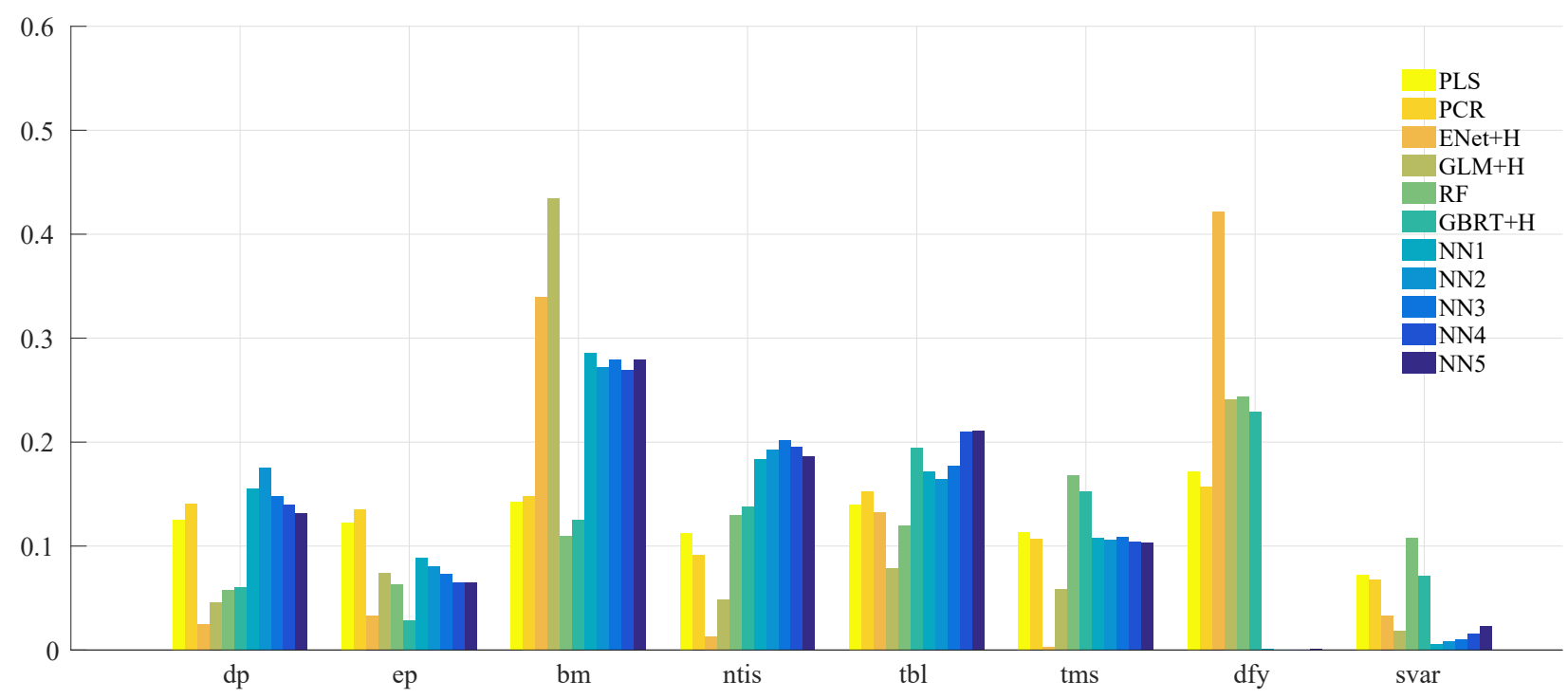

Note: Variable importance for eight macroeconomic variables in each model. Variable importance is an average over all training samples. Variable importances within each model are normalized to sum to one. The lower panel provides a complementary visual comparison of macroeconomic variable importances.

methods provide a highly consistent summary of which variables are most influential for forecast accuracy. The full set of SSD results are shown in appendix Figure A.2.

For robustness, we re-run our analysis with an augmented set of characteristics that include five placebo "characteristics." They are simulated according to the data generating process (A.1) in Appendix A. The parameters are calibrated to have similar behavior as our characteristics dataset but are independent of future returns by construction. Figure A.3 in Appendix F presents the variable importance plot (based on $R^{2}$ ) with five noise characteristics highlighted. The table confirms that the most influential characteristics that we identify in our main analysis are unaffected by the presence of irrelevant characteristics. Noise variables appear among the least informative characteristics, along with sin stocks, dividend initiation/omission, cashflow volatility, and other accounting variables.

Table 4 shows the $R^{2}$-based importance measure for each macroeconomic predictor variable (again normalized to sum to one within a given model). All models agree that the aggregate book-to-market ratio is a critical predictor, whereas market volatility has little role in any model. PLS and PCR place similar weights on all other predictors, potentially because these variables are highly correlated. Linear and generalized linear models strongly favor bond market variables including the default 
spread and treasury rate. Nonlinear methods (trees and neural networks) place great emphasis on exactly those predictors ignored by linear methods, such as term spreads and issuance activity.

Many accounting characteristics are not available at the monthly frequency, which might explain their low importance in Figure 5. To investigate this, appendix Figure A.6 presents the rank of variables based on the annual return forecasts. Price trend variables become less important compared to the liquidity and risk measures, although they are still quite influential. The characteristics that were ranked in the bottom half of predictors at the monthly horizon remain largely unimportant at the annual horizon. The exception is industry (sic2) which shows substantial predictive power at the annual frequency.

\subsubsection{Marginal Association Between Characteristics and Expected Returns}

Figure 6 traces out the model-implied marginal impact of individual characteristics on expected excess returns. Our data transformation normalizes characteristics to the $(-1,1)$ interval, and holds all other variables fixed at their median value of zero. We choose four illustrative characteristics for the figure, including size (mvel1), momentum (mom12m), stock volatility (retvol), and accruals (acc).

First, Figure 6 illustrates that machine learning methods identify patterns similar to some well known empirical phenomena. For example, expected stock returns are decreasing in size, increasing in past one-year return, and decreasing in stock volatility. And it is interesting to see that all methods agree on a nearly exact zero relationship between accruals and future returns. Second, the (penalized) linear model finds no predictive association between returns and either size or volatility, while trees and neural networks find large sensitivity of expected returns to both of these variables. For example, a firm that drops from median size to the $20^{\text {th }}$ percentile of the size distribution experiences an increase in its annualized expected return of roughly $2.4 \%(0.002 \times 12 \times 100)$, and a firm whose volatility rises from median to $80^{\text {th }}$ percentile experiences a decrease of around $3.0 \%$ per year, according to NN3, and these methods detect nonlinear predictive associations. The inability of linear models to capture nonlinearities can lead them to prefer a zero association, and this can in part explain the divergence in the performance of linear and nonlinear methods.

\subsubsection{Interaction Effects}

The favorable performance of trees and neural networks indicates a benefit to allowing for potentially complex interactions among predictors. Machine learning models are often referred to as "black boxes." This is in some sense a misnomer, as the models are readily inspectable. They are, however, complex, and this is the source of both their power and their opacity. Any exploration of interaction effect is vexed by vast possibilities for identity and functional forms for interacting predictors. In this section, we present a handful of interaction results to help illustrate the inner workings of one black box method, the NN3 model.

As a first example, we examine a set of pairwise interaction effects in NN3. Figure 7 reports how expected returns vary as we simultaneously vary values of a pair of characteristics over their support 
Figure 6: Marginal Association Between Expected Returns and Characteristics
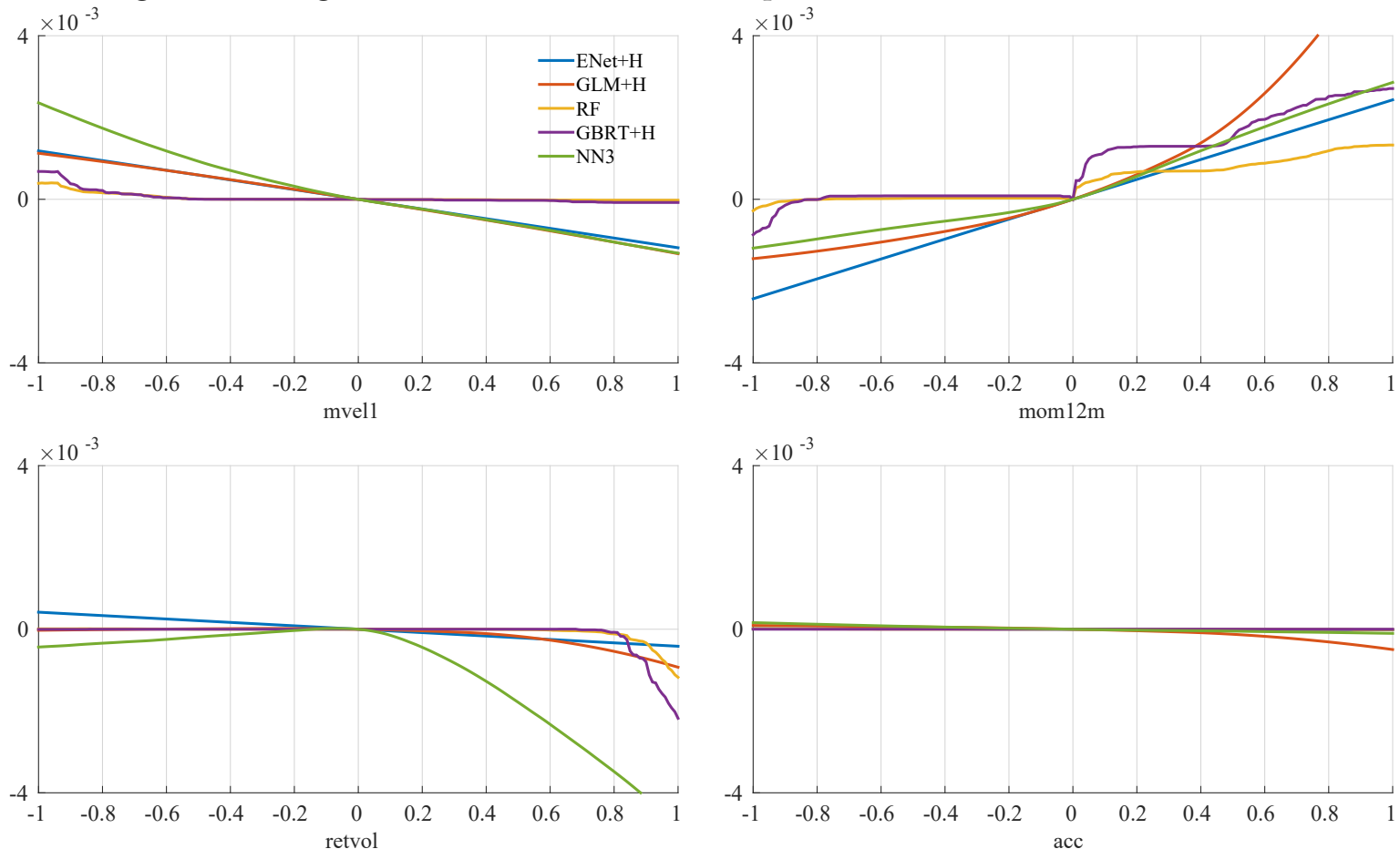

Note: Sensitivity of expected monthly percentage returns (vertical axis) to individual characteristics (holding all other covariates fixed at their median values).

$[-1,1]$, while holding all other variables fixed at their median value of zero. We show interactions of stock size (mvel1) with four other predictors: short-term reversal (mom1m), momentum (mom12m), and total and idiosyncratic volatility (retvol and idiovol, respectively).

The upper-left figure shows that the short-term reversal effect is strongest and is essentially linear among small stocks (blue line). Among large stocks (green line), reversal is concave, occurring primarily when the prior month return is positive. The upper-right figure shows the momentum effect, which is most pronounced among large stocks for the NN3 model. ${ }^{37}$ Likewise, on the lowerleft, we see that the low volatility anomaly is also strongest among large stocks. For small stocks, the volatility effect is hump-shaped. Finally, the lower-right shows that NN3 estimates no interaction effect between size and accruals - the size lines are simply vertical shifts of the univariate accruals curve.

Figure 8 illustrates interactions between stock-level characteristics and macroeconomic indicator variables. It shows, for example, that the size effect is more pronounced when aggregate valuations are low (bm is high) and when equity issuance (ntis) is low, while the low volatility anomaly is especially strong in high valuation and high issuance environments. Appendix Figure A.4 shows the 100 most important interactions of stock characteristics with macroeconomic indicators for each machine learning model. The most influential features come from interacting a stock's recent price

\footnotetext{
${ }^{37}$ Note that conclusions from our model can diverge from results in the literature because we jointly model hundreds of predictor variables, which can lead to new conclusions regarding marginal effects, interaction effects, and so on.
} 
Figure 7: Expected Returns and Characteristic Interactions (NN3)
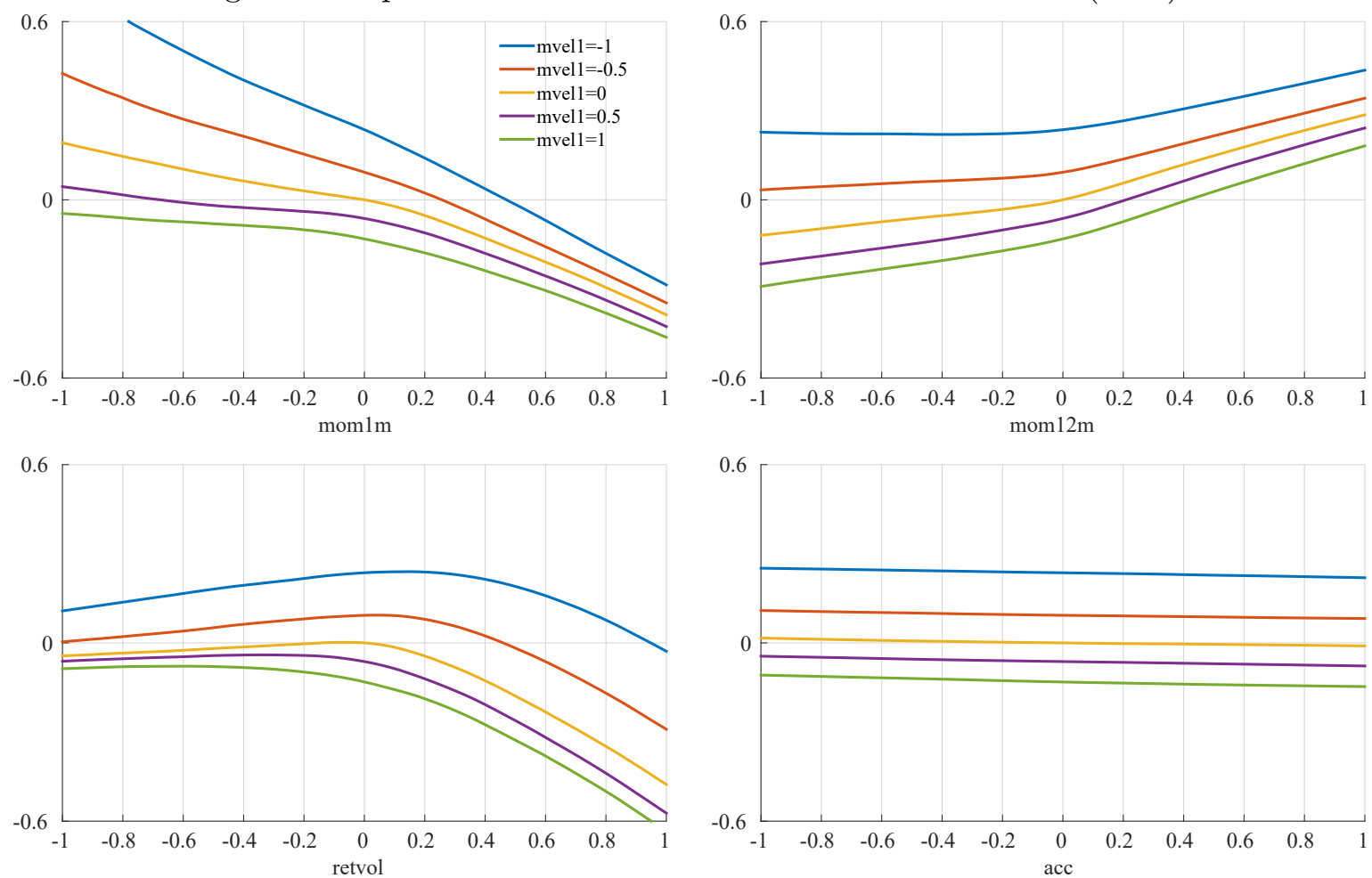

Note: Sensitivity of expected monthly percentage returns (vertical axis) to interactions effects for mvel1 with mom $1 \mathrm{~m}$, mom12m, retvol, and acc in model NN3 (holding all other covariates fixed at their median values).

trends (e.g., momentum, short-term reversal, or industry momentum) with aggregate asset price levels (e.g., valuation ratios of the aggregate stock market or Treasury bill rates). Furthermore, the dominant macroeconomic interactions are stable over time, as illustrated in appendix Figure A.5.

\subsection{Portfolio Forecasts}

So far we have analyzed predictability of individual stock returns. Next, we compare forecasting performance of machine learning methods for aggregate portfolio returns. There are a number of benefits to analyzing portfolio-level forecasts.

First, because all of our models are optimized for stock-level forecasts, portfolio forecasts provide an additional indirect evaluation of the model and its robustness. Second, aggregate portfolios tend to be of broader economic interest because they represent the risky-asset savings vehicles most commonly held by investors (via mutual funds, ETFs, and hedge funds). We study value-weight portfolios to assess the extent to which a model's predictive performance thrives in the most valuable (and most economically important) assets in the economy. Third, the distribution of portfolio returns is sensitive to dependence among stock returns, with the implication that a good stock-level prediction model is not guaranteed to produce accurate portfolio-level forecasts. Bottom-up portfolio forecasts allow us to evaluate a model's ability to transport its asset predictions, which occur at the finest 
Figure 8: Expected Returns and Characteristic/Macroeconomic Variable Interactions (NN3)
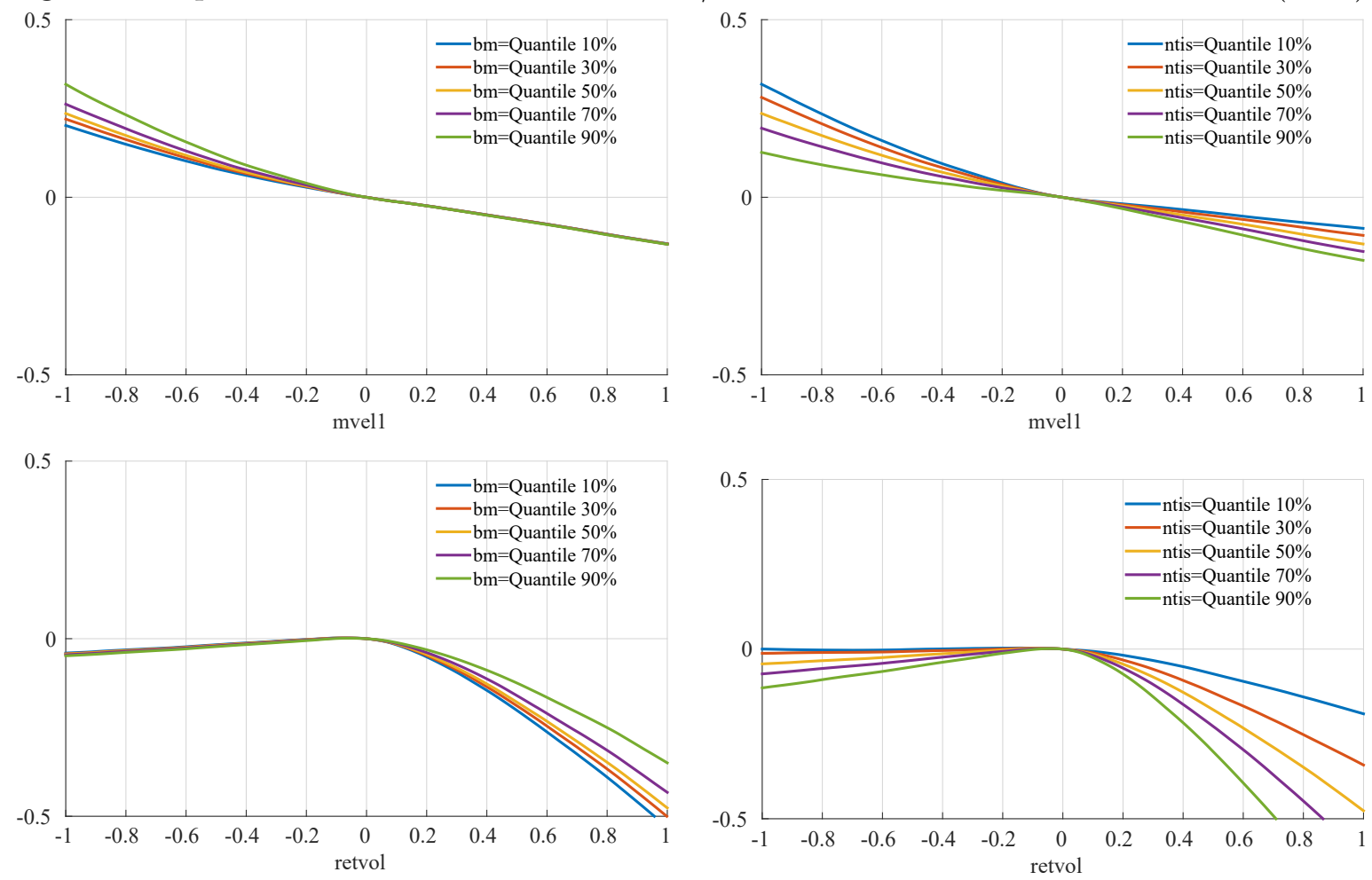

Note: Sensitivity of expected monthly percentage returns (vertical axis) to interactions effects for mvel1 and retvol with bm and ntis in model NN3 (holding all other covariates fixed at their median values).

asset level, into broader investment contexts. Last but not least, the portfolio results are one step further "out-of-sample" in that the optimization routine does not directly account for the predictive performance of the portfolios.

Our assessment of forecast performance up to this point has been entirely statistical, relying on comparisons of predictive $R^{2}$. The final advantage of analyzing predictability at the portfolio level is that we can assess the economic contribution of each method via its contribution to risk-adjusted portfolio return performance.

\subsubsection{Pre-specified Portfolios}

We build bottom-up forecasts by aggregating individual stock return predictions into portfolios. Given the weight of stock $i$ in portfolio $p$ (denoted $w_{i, t}^{p}$ ) and given a model-based out-of-sample forecast for stock $i$ (denoted $\widehat{r}_{i, t+1}$ ), we construct the portfolio return forecast as

$$
\widehat{r}_{t+1}^{p}=\sum_{i=1}^{n} w_{i, t}^{p} \times \widehat{r}_{i, t+1} .
$$

This bottom-up approach works for any target portfolio whose weights are known a priori.

We form bottom-up forecasts for 30 of the most well known portfolios in the empirical finance 
Table 5: Monthly Portfolio-level Out-of-Sample Predictive $R^{2}$

\begin{tabular}{|c|c|c|c|c|c|c|c|c|c|c|c|c|}
\hline & $\begin{array}{c}\text { OLS-3 } \\
+\mathrm{H}\end{array}$ & PLS & PCR & $\begin{array}{c}\text { ENet } \\
+\mathrm{H}\end{array}$ & $\begin{array}{c}\text { GLM } \\
+\mathrm{H}\end{array}$ & $\mathrm{RF}$ & $\begin{array}{c}\text { GBRT } \\
+\mathrm{H}\end{array}$ & NN1 & NN2 & NN3 & NN4 & NN5 \\
\hline \multicolumn{13}{|c|}{ Panel A: Common Factor Portfolios } \\
\hline S\&P 500 & -0.22 & -0.86 & -1.55 & 0.75 & 0.71 & 1.37 & 1.40 & 1.08 & 1.13 & 1.80 & 1.63 & 1.17 \\
\hline SMB & 0.81 & 2.09 & 0.39 & 1.72 & 2.36 & 0.57 & 0.35 & 1.40 & 1.16 & 1.31 & 1.20 & 1.27 \\
\hline HML & 0.66 & 0.50 & 1.21 & 0.46 & 0.84 & 0.98 & 0.21 & 1.22 & 1.31 & 1.06 & 1.25 & 1.24 \\
\hline RMW & -2.35 & 1.19 & 0.41 & -1.07 & -0.06 & -0.54 & -0.92 & 0.68 & 0.47 & 0.84 & 0.53 & 0.54 \\
\hline CMA & 0.80 & -0.44 & 0.03 & -1.07 & 1.24 & -0.11 & -1.04 & 1.88 & 1.60 & 1.06 & 1.84 & 1.31 \\
\hline UMD & -0.90 & -1.09 & -0.47 & 0.47 & -0.37 & 1.37 & -0.25 & -0.56 & -0.26 & 0.19 & 0.27 & 0.35 \\
\hline \multicolumn{13}{|c|}{ Panel B: Sub-components of Factor Portfolios } \\
\hline Big Value & 0.10 & 0.00 & -0.33 & 0.25 & 0.59 & 1.31 & 1.06 & 0.85 & 0.87 & 1.46 & 1.21 & 0.99 \\
\hline Big Growth & -0.33 & -1.26 & -1.62 & 0.70 & 0.51 & 1.32 & 1.19 & 1.00 & 1.10 & 1.50 & 1.24 & 1.11 \\
\hline Big Neutral & -0.17 & -1.09 & -1.51 & 0.80 & 0.36 & 1.31 & 1.28 & 1.43 & 1.24 & 1.70 & 1.81 & 1.40 \\
\hline Small Value & 0.30 & 1.66 & 1.05 & 0.64 & 0.85 & 1.24 & 0.52 & 1.59 & 1.37 & 1.54 & 1.40 & 1.30 \\
\hline Small Growth & -0.16 & 0.14 & -0.18 & -0.33 & -0.12 & 0.71 & 1.24 & 0.05 & 0.42 & 0.48 & 0.41 & 0.50 \\
\hline Small Neutral & -0.27 & 0.60 & 0.19 & 0.21 & 0.28 & 0.88 & 0.36 & 0.58 & 0.62 & 0.70 & 0.58 & 0.68 \\
\hline Big Conservative & -0.57 & -0.10 & -1.06 & 1.02 & 0.46 & 1.11 & 0.55 & 1.15 & 1.13 & 1.59 & 1.37 & 1.07 \\
\hline Big Aggressive & 0.20 & -0.80 & -1.15 & 0.30 & 0.67 & 1.75 & 2.00 & 1.33 & 1.51 & 1.78 & 1.55 & 1.42 \\
\hline Big Neutral & -0.29 & -1.75 & -1.96 & 0.83 & 0.48 & 1.13 & 0.77 & 0.85 & 0.85 & 1.51 & 1.45 & 1.16 \\
\hline Small Conservative & -0.05 & 1.17 & 0.71 & -0.02 & 0.34 & 0.96 & 0.56 & 0.82 & 0.87 & 0.96 & 0.90 & 0.83 \\
\hline Small Aggressive & -0.10 & 0.51 & 0.01 & -0.09 & 0.14 & 1.00 & 1.46 & 0.34 & 0.64 & 0.75 & 0.62 & 0.71 \\
\hline Small Neutral & -0.30 & 0.45 & 0.12 & 0.42 & 0.35 & 0.76 & -0.01 & 0.70 & 0.69 & 0.83 & 0.66 & 0.72 \\
\hline Big Robust & -1.02 & -1.08 & -2.06 & 0.55 & 0.35 & 1.1 & 0.33 & 0.74 & 0.79 & 1.28 & 1.03 & 0.74 \\
\hline Big Weak & -0.12 & 1.42 & 1.07 & 0.89 & 1.10 & 1.33 & 1.77 & 1.79 & 1.79 & 2.05 & 1.66 & 1.60 \\
\hline Big Neutral & 0.86 & -1.22 & -1.26 & 0.41 & 0.13 & 1.10 & 0.91 & 0.84 & 0.94 & 1.19 & 1.15 & 0.99 \\
\hline Small Robust & -0.71 & 0.35 & -0.38 & -0.04 & -0.42 & 0.70 & 0.19 & 0.24 & 0.50 & 0.63 & 0.53 & 0.55 \\
\hline Small Weak & 0.05 & 1.06 & 0.59 & -0.13 & 0.44 & 1.05 & 1.42 & 0.71 & 0.92 & 0.99 & 0.90 & 0.89 \\
\hline Small Neutral & -0.51 & 0.07 & -0.47 & -0.33 & -0.32 & 0.60 & -0.08 & 0.10 & 0.25 & 0.38 & 0.32 & 0.41 \\
\hline Big Up & 0.20 & -0.25 & -1.24 & 0.66 & 1.17 & 1.18 & 0.90 & 0.80 & 0.76 & 1.13 & 1.12 & 0.93 \\
\hline Big Down & -1.54 & -1.63 & -1.55 & 0.44 & -0.33 & 1.14 & 0.71 & 0.36 & 0.70 & 1.07 & 0.90 & 0.84 \\
\hline Big Medium & -0.04 & -1.51 & -1.94 & 0.81 & -0.08 & 1.57 & 1.80 & 1.29 & 1.32 & 1.71 & 1.55 & 1.23 \\
\hline Small Up & 0.07 & 0.78 & 0.56 & -0.07 & 0.25 & 0.62 & -0.03 & 0.06 & 0.07 & 0.21 & 0.19 & 0.25 \\
\hline Small Down & -0.21 & 0.15 & -0.20 & 0.15 & -0.01 & 1.51 & 1.38 & 0.74 & 0.82 & 1.02 & 0.91 & 0.96 \\
\hline Small Medium & 0.07 & 0.82 & 0.20 & 0.59 & 0.37 & 1.22 & 1.06 & 1.09 & 1.09 & 1.18 & 1.00 & 1.03 \\
\hline
\end{tabular}

Note: In this table, we report the out-of-sample predictive $R^{2}$ s for 30 portfolios using OLS with size, book-to-market, and momentum, OLS-3, PLS, PCR, elastic net (ENet), generalized linear model with group lasso (GLM), random forest $(\mathrm{RF})$, gradient boosted regression trees (GBRT), and five architectures of neural networks (NN1,..,NN5), respectively. " $+\mathrm{H}$ " indicates the use of Huber loss instead of the $l_{2}$ loss. The six portfolios in Panel A are the S\&P 500 index and the Fama-French SMB, HML, CMA, RMW, and UMD factors. The 24 portfolios in Panel B are $3 \times 2$ size double-sorted portfolios used in the construction of the Fama-French value, investment, profitability, and momentum factors.

literature, including the S\&P 500, the Fama-French size, value, profitability, investment, and momentum factor portfolios (SMB, HML, RMW, CMA, and UMD, respectively), and sub-components of these Fama-French portfolios, including six size and value portfolios, six size and investment portfolios, six size and profitability portfolios, and six size and momentum portfolios. The sub-component portfolios are long-only and therefore have substantial overlap with the market index, while SMB, HML, RMW, CMA, and UMD are zero-net-investment long-short portfolios that are in large part 
Table 6: Market Timing Sharpe Ratio Gains

\begin{tabular}{|c|c|c|c|c|c|c|c|c|c|c|c|c|}
\hline & $\begin{array}{c}\text { OLS-3 } \\
+\mathrm{H}\end{array}$ & PLS & PCR & $\begin{array}{c}\text { ENet } \\
+\mathrm{H}\end{array}$ & $\begin{array}{c}\text { GLM } \\
+\mathrm{H}\end{array}$ & $\mathrm{RF}$ & $\begin{array}{c}\text { GBRT } \\
+\mathrm{H}\end{array}$ & NN1 & NN2 & NN3 & NN4 & NN5 \\
\hline \multicolumn{13}{|c|}{ Panel A: Common Factor Portfolios } \\
\hline S\&P 500 & 0.07 & 0.05 & -0.06 & 0.12 & 0.19 & 0.18 & 0.19 & 0.22 & 0.20 & 0.26 & 0.22 & 0.19 \\
\hline SMB & 0.06 & 0.17 & 0.09 & 0.24 & 0.26 & 0.00 & -0.07 & 0.21 & 0.18 & 0.15 & 0.09 & 0.11 \\
\hline HML & 0.00 & 0.01 & 0.04 & -0.03 & -0.02 & 0.04 & 0.02 & 0.04 & 0.06 & 0.04 & 0.02 & 0.01 \\
\hline RMW & 0.00 & -0.01 & -0.06 & -0.19 & -0.13 & -0.11 & -0.01 & -0.03 & -0.09 & 0.01 & 0.01 & -0.07 \\
\hline CMA & 0.02 & 0.02 & 0 & -0.09 & -0.05 & 0.08 & -0.01 & 0.00 & 0.01 & 0.05 & 0.04 & 0.06 \\
\hline UMD & 0.01 & -0.06 & -0.02 & -0.02 & -0.07 & -0.04 & -0.07 & -0.04 & -0.08 & -0.04 & -0.10 & -0.01 \\
\hline \multicolumn{13}{|c|}{ Panel B: Sub-components of Factor Portfolios } \\
\hline Big Value & -0.01 & 0.06 & -0.03 & 0.09 & 0.06 & 0.09 & 0.08 & 0.11 & 0.11 & 0.13 & 0.10 & 0.11 \\
\hline Big Growth & 0.08 & -0.01 & -0.08 & 0.10 & 0.17 & 0.20 & 0.21 & 0.22 & 0.20 & 0.26 & 0.22 & 0.21 \\
\hline Big Neutral & 0.06 & 0.03 & -0.06 & 0.11 & 0.16 & 0.13 & 0.17 & 0.23 & 0.21 & 0.23 & 0.23 & 0.21 \\
\hline Small Value & -0.04 & 0.15 & 0.09 & 0.01 & 0.08 & 0.07 & 0.08 & 0.11 & 0.11 & 0.10 & 0.11 & 0.13 \\
\hline Small Growth & 0.00 & 0.03 & -0.06 & -0.03 & -0.05 & 0.04 & 0.05 & 0.02 & 0.03 & 0.03 & 0.02 & 0.02 \\
\hline Small Neutral & 0.02 & 0.09 & 0.05 & 0.03 & 0.04 & 0.11 & 0.11 & 0.09 & 0.08 & 0.10 & 0.09 & 0.11 \\
\hline Big Conservative & 0.08 & 0.02 & -0.04 & 0.08 & 0.15 & 0.09 & 0.13 & 0.17 & 0.14 & 0.19 & 0.16 & 0.14 \\
\hline Big Aggressive & 0.08 & -0.01 & -0.11 & 0.01 & 0.13 & 0.22 & 0.18 & 0.21 & 0.19 & 0.23 & 0.20 & 0.20 \\
\hline Big Neutral & 0.04 & -0.01 & -0.08 & 0.09 & 0.11 & 0.09 & 0.11 & 0.13 & 0.12 & 0.18 & 0.18 & 0.16 \\
\hline Small Conservative & 0.04 & 0.17 & 0.12 & 0.02 & 0.05 & 0.17 & 0.15 & 0.11 & 0.11 & 0.14 & 0.13 & 0.15 \\
\hline Small Aggressive & 0.01 & 0.05 & -0.06 & -0.05 & -0.03 & 0.08 & 0.06 & 0.02 & 0.05 & 0.06 & 0.04 & 0.05 \\
\hline Small Neutral & 0.01 & 0.06 & 0.03 & 0.01 & 0.04 & 0.08 & 0.09 & 0.07 & 0.06 & 0.08 & 0.07 & 0.09 \\
\hline Big Robust & 0.10 & 0.07 & -0.07 & 0.11 & 0.18 & 0.17 & 0.18 & 0.18 & 0.16 & 0.22 & 0.19 & 0.16 \\
\hline Big Weak & 0.05 & 0.12 & 0.05 & 0.09 & 0.12 & 0.21 & 0.17 & 0.22 & 0.20 & 0.21 & 0.18 & 0.19 \\
\hline Big Neutral & 0.09 & 0.00 & -0.04 & 0.09 & 0.20 & 0.19 & 0.17 & 0.22 & 0.21 & 0.24 & 0.21 & 0.20 \\
\hline Small Robust & 0.09 & 0.04 & -0.03 & 0.00 & 0.00 & 0.10 & 0.07 & 0.04 & 0.05 & 0.08 & 0.08 & 0.08 \\
\hline Small Weak & -0.03 & 0.09 & 0.00 & -0.03 & -0.02 & 0.07 & 0.07 & 0.06 & 0.06 & 0.06 & 0.05 & 0.06 \\
\hline Small Neutral & 0.04 & 0.04 & -0.03 & 0.00 & 0.01 & 0.11 & 0.09 & 0.04 & 0.04 & 0.07 & 0.07 & 0.08 \\
\hline Big Up & 0.10 & 0.05 & -0.06 & 0.10 & 0.21 & 0.16 & 0.14 & 0.17 & 0.14 & 0.17 & 0.18 & 0.17 \\
\hline Big Down & -0.02 & 0.09 & -0.08 & -0.02 & 0.02 & 0.08 & 0.10 & 0.10 & 0.07 & 0.12 & 0.11 & 0.09 \\
\hline Big Medium & -0.01 & 0.04 & -0.06 & 0.14 & 0.09 & 0.17 & 0.20 & 0.22 & 0.21 & 0.25 & 0.22 & 0.19 \\
\hline Small Up & 0.08 & 0.13 & 0.10 & 0.05 & 0.07 & 0.16 & 0.12 & 0.07 & 0.06 & 0.08 & 0.07 & 0.10 \\
\hline Small Down & -0.14 & 0.04 & -0.05 & -0.09 & -0.05 & 0.06 & 0.04 & 0.01 & 0.01 & 0.02 & 0.01 & 0.01 \\
\hline Small Medium & 0.05 & 0.11 & 0.07 & 0.08 & 0.09 & 0.13 & 0.15 & 0.13 & 0.12 & 0.14 & 0.13 & 0.15 \\
\hline
\end{tabular}

Note: Improvement in annualized Sharpe ratio $\left(S R^{*}-S R\right)$. We compute the $S R^{*}$ by weighting the portfolios based on a market timing strategy Campbell and Thompson (2007). Cases with Sharpe ratio deterioration are omitted.

purged of market exposure. In all cases, we create the portfolios ourselves using CRSP market equity value weights. Our portfolio construction differs slightly from the actual S\&P 500 index and the characteristic-based Fama-French portfolios (Fama and French, 1993, 2015), but has the virtue that we can exactly track the ex ante portfolio weights of each. ${ }^{38}$

Table 5 reports the monthly out-of-sample $R^{2}$ over our 30-year testing sample. Regularized linear methods fail to outperform naive constant forecasts of the S\&P 500. In contrast, all nonlinear models have substantial positive predictive performance. The one month out-of-sample $R^{2}$ is $0.71 \%$ for the

\footnotetext{
${ }^{38}$ Our replication of S\&P500 returns has a correlation with the actual index of more than 0.99. For the other portfolios (SMB, HML, RMW, CMA, UMD), the return correlations between our replication and the version from Ken French's website are $0.99,0.97,0.95,0.99,0.96$, respectively.
} 
generalized linear model and reaches as high as $1.80 \%$ for the three-layer neural network. As a benchmark for comparison, nearly all of the macroeconomic return predictor variables in the survey of Welch and Goyal (2008) fail to produce a positive out-of-sample forecast $R^{2}$. Kelly and Pruitt (2013) find that PLS delivers an out-of-sample forecasting $R^{2}$ around $1 \%$ per month for the aggregate market index, though their forecasts directly target the market return as opposed to being bottomup forecasts. And the most well-studied portfolio predictors, such as the aggregate price-dividend ratio, typically produce an in-sample predictive $R^{2}$ of around $1 \%$ per month (e.g., Cochrane, 2007), smaller than what we find out-of-sample.

The patterns in S\&P 500 forecasting performance across models carry over to long-only characteristicsorted portfolios (rows 2-25) and long-short factor portfolios (SMB, HML, RMW, CMA, and UMD). Nonlinear methods excel. NN3-NN5 produce a positive $R_{\text {oos }}^{2}$ for every portfolio analyzed, with NN3 dominating. NN1 and NN2 also produce a positive $R_{\mathrm{oos}}^{2}$ for all but UMD (which is by and large the hardest strategy to forecast). The generalized linear model $R_{\text {oos }}^{2}$ is positive for 22 out of 30 portfolios. Linear methods, on the other hand, are on balance unreliable for bottom-up portfolio return forecasting, though their performance tends to be better for "big" portfolios compared to "small." For select long-short factor portfolios (e.g., SMB), constrained linear methods such as PLS perform comparatively well (consistent with the findings of Kelly and Pruitt, 2013). In short, machine learning methods, and nonlinear methods in particular, produce unusually powerful out-of-sample portfolio predictions.

We next assess the economic magnitudes of portfolio predictability. Campbell and Thompson (2008) show that small improvements in $R^{2}$ can map into large utility gains for a mean-variance investor. They show that the Sharpe ratio $\left(S R^{*}\right)$ earned by an active investor exploiting predictive information (summarized as a predictive $R^{2}$ ) improves over the Sharpe ratio $(S R)$ earned by a buy-and-hold investor according to

$$
S R^{*}=\sqrt{\frac{S R^{2}+R^{2}}{1-R^{2}}}
$$

We use this formula to translate the predictive $R_{\text {oos }}^{2}$ of Table 5 (along with the full-sample Sharpe ratio of each portfolio) into an improvement in annualized Sharpe ratio, $S R^{*}-S R$, for an investor exploiting machine learning predictions for portfolio timing. The results are presented in appendix Table A.7. For example, the buy-and-hold Sharpe ratio of the S\&P 500, which is 0.51 in our 30-year out-of-sample period, can be improved to 0.71 by a market-timer exploiting forecasts from the NN3 model. For characteristic-based portfolios, nonlinear machine learning methods help improve Sharpe ratios by anywhere from a few percentage points to over 24 percentage points.

Campbell and Thompson (2008) also propose evaluating the economic magnitude of portfolio predictability with a market timing trading strategy. We follow their out-of-sample trading strategy exactly, scaling up/down positions each month as expected returns rise/fall, while imposing a maximum leverage constraint of $50 \%$ and excluding short sales for long-only portfolios (we do not impose these constraints for long-short factor portfolios). Table 6 reports the annualized Sharpe ratio gains (relative to a buy-and-hold strategy) for timing strategies based on machine learning forecasts. Con- 
sistent with our other results, the strongest and most consistent trading strategies are those based on nonlinear models, with neural networks the best overall. In the case of NN3, the Sharpe ratio from timing the S\&P 500 index 0.77 , or 26 percentage points higher than a buy-and-hold position. Longshort factor portfolios are harder to time than the S\&P 500 and the other 24 long-only portfolios, and all methods fail to outperform the static UMD strategy.

As a robustness test, we also analyze bottom-up predictions for annual rather than monthly returns. The comparative patterns in predictive performance across methods is the same in annual and monthly data. Appendix Table A.8 demonstrates the superiority of nonlinear methods, and in particular neural networks. NN3 continues to dominate for the market portfolio, achieving an annual $R_{\text {oos }}^{2}$ of $15.7 \%$.

\subsubsection{Machine Learning Portfolios}

Next, rather than assessing forecast performance among pre-specified portfolios, we design a new set of portfolios to directly exploit machine learning forecasts. At the end of each month, we calculate one-month-ahead out-of-sample stock return predictions for each method. We then sort stocks into deciles based on each model's forecasts. We reconstitute portfolios each month using value weights. Finally, we construct a zero-net-investment portfolio that buys the highest expected return stocks (decile 10) and sells the lowest (decile 1).

Table 7 reports results. Out-of-sample portfolio performance aligns very closely with results on machine learning forecast accuracy reported earlier. Realized returns generally increase monotonically with machine learning forecasts from every method (with occasional exceptions, such as decile 8 of NN1). Neural network models again dominate linear models and tree-based approaches. In particular, for all but the most extreme deciles, the quantitative match between predicted returns and average realized returns using neural networks is extraordinarily close. The best 10-1 strategy comes from NN4, which returns on average $2.3 \%$ per month (27.1\% on an annualized basis). Its monthly volatility is $5.8 \%$ (20.1\% annualized), amounting to an annualized out-of-sample Sharpe ratio of 1.35 .

While value-weight portfolios are less sensitive to trading cost considerations, it is perhaps more natural to study equal weights in our analysis because our statistical objective functions minimize equally weighted forecast errors. Appendix Table A.9 reports the performance of machine learning portfolios using an equal-weight formation. The qualitative conclusions of this table are identical to those of Table 7, but the Sharpe ratios are substantially higher. For example, the long-short decile spread portfolio based on the NN4 model earns an annualized Sharpe ratio of 2.45 with equal weighting. To identify the extent to which equal-weight results are driven by micro-cap stocks, Table A.10 reports equal-weight portfolio results excluding stocks that fall below the $20^{\text {th }}$ percentile of the NYSE size distribution. In this case, the NN4 long-short decile spread earns a Sharpe ratio of 1.69.

As recommended by Lewellen (2015), the OLS-3 model is an especially parsimonious and robust benchmark model. He also recommends somewhat larger OLS benchmark models with either seven or 15 predictors, which we report in appendix Table A.11. The larger OLS models improve over 
Table 7: Performance of Machine Learning Portfolios

\begin{tabular}{|c|c|c|c|c|c|c|c|c|c|c|c|c|}
\hline & \multicolumn{4}{|c|}{ OLS-3+H } & \multicolumn{4}{|c|}{ PLS } & \multicolumn{4}{|c|}{ PCR } \\
\hline & Pred & Avg & Std & SR & Pred & Avg & Std & SR & Pred & Avg & Std & $\mathrm{SR}$ \\
\hline $\operatorname{Low}(\mathrm{L})$ & -0.17 & 0.40 & 5.90 & 0.24 & -0.83 & 0.29 & 5.31 & 0.19 & -0.68 & 0.03 & 5.98 & 0.02 \\
\hline 2 & 0.17 & 0.58 & 4.65 & 0.43 & -0.21 & 0.55 & 4.96 & 0.38 & -0.11 & 0.42 & 5.25 & 0.28 \\
\hline 3 & 0.35 & 0.60 & 4.43 & 0.47 & 0.12 & 0.64 & 4.63 & 0.48 & 0.19 & 0.53 & 4.94 & 0.37 \\
\hline 4 & 0.49 & 0.71 & 4.32 & 0.57 & 0.38 & 0.78 & 4.30 & 0.63 & 0.42 & 0.68 & 4.64 & 0.51 \\
\hline 5 & 0.62 & 0.79 & 4.57 & 0.60 & 0.61 & 0.77 & 4.53 & 0.59 & 0.62 & 0.81 & 4.66 & 0.60 \\
\hline 6 & 0.75 & 0.92 & 5.03 & 0.63 & 0.84 & 0.88 & 4.78 & 0.64 & 0.81 & 0.81 & 4.58 & 0.61 \\
\hline 7 & 0.88 & 0.85 & 5.18 & 0.57 & 1.06 & 0.92 & 4.89 & 0.65 & 1.01 & 0.87 & 4.72 & 0.64 \\
\hline 8 & 1.02 & 0.86 & 5.29 & 0.56 & 1.32 & 0.92 & 5.14 & 0.62 & 1.23 & 1.01 & 4.77 & 0.73 \\
\hline 9 & 1.21 & 1.18 & 5.47 & 0.75 & 1.66 & 1.15 & 5.24 & 0.76 & 1.52 & 1.20 & 4.88 & 0.86 \\
\hline $\operatorname{High}(\mathrm{H})$ & 1.51 & 1.34 & 5.88 & 0.79 & 2.25 & 1.30 & 5.85 & 0.77 & 2.02 & 1.25 & 5.60 & 0.77 \\
\hline \multirow[t]{3}{*}{$\mathrm{H}-\mathrm{L}$} & 1.67 & 0.94 & 5.33 & 0.61 & 3.09 & 1.02 & 4.88 & 0.72 & 2.70 & 1.22 & 4.82 & 0.88 \\
\hline & \multicolumn{4}{|c|}{ ENet $+\mathrm{H}$} & \multicolumn{4}{|c|}{$\mathrm{GLM}+\mathrm{H}$} & \multicolumn{4}{|c|}{$\mathrm{RF}$} \\
\hline & Pred & Avg & Std & $\mathrm{SR}$ & Pred & Avg & Std & $\mathrm{SR}$ & Pred & Avg & Std & $\mathrm{SR}$ \\
\hline $\operatorname{Low}(\mathrm{L})$ & -0.04 & 0.24 & 5.44 & 0.15 & -0.47 & 0.08 & 5.65 & 0.05 & 0.29 & -0.09 & 6.00 & -0.05 \\
\hline 2 & 0.27 & 0.56 & 4.84 & 0.40 & 0.01 & 0.49 & 4.80 & 0.35 & 0.44 & 0.38 & 5.02 & 0.27 \\
\hline 3 & 0.44 & 0.53 & 4.50 & 0.40 & 0.29 & 0.65 & 4.52 & 0.50 & 0.53 & 0.64 & 4.70 & 0.48 \\
\hline 4 & 0.59 & 0.72 & 4.11 & 0.61 & 0.50 & 0.72 & 4.59 & 0.55 & 0.60 & 0.60 & 4.56 & 0.46 \\
\hline 5 & 0.73 & 0.72 & 4.42 & 0.57 & 0.68 & 0.70 & 4.55 & 0.53 & 0.67 & 0.57 & 4.51 & 0.44 \\
\hline 6 & 0.87 & 0.85 & 4.60 & 0.64 & 0.84 & 0.84 & 4.53 & 0.65 & 0.73 & 0.64 & 4.54 & 0.49 \\
\hline 7 & 1.01 & 0.87 & 4.75 & 0.64 & 1.00 & 0.86 & 4.82 & 0.62 & 0.80 & 0.67 & 4.65 & 0.50 \\
\hline 8 & 1.16 & 0.88 & 5.20 & 0.59 & 1.18 & 0.87 & 5.18 & 0.58 & 0.87 & 1.00 & 4.91 & 0.71 \\
\hline 9 & 1.36 & 0.80 & 5.61 & 0.50 & 1.40 & 1.04 & 5.44 & 0.66 & 0.96 & 1.23 & 5.59 & 0.76 \\
\hline $\operatorname{High}(\mathrm{H})$ & 1.66 & 0.84 & 6.76 & 0.43 & 1.81 & 1.14 & 6.33 & 0.62 & 1.12 & 1.53 & 7.27 & 0.73 \\
\hline \multirow[t]{3}{*}{$\mathrm{H}-\mathrm{L}$} & 1.70 & 0.60 & 5.37 & 0.39 & 2.27 & 1.06 & 4.79 & 0.76 & 0.83 & 1.62 & 5.75 & 0.98 \\
\hline & \multicolumn{4}{|c|}{$\mathrm{GBRT}+\mathrm{H}$} & \multicolumn{4}{|c|}{ NN1 } & \multicolumn{4}{|c|}{$\mathrm{NN} 2$} \\
\hline & Pred & Avg & Std & $\mathrm{SR}$ & Pred & Avg & Std & $\mathrm{SR}$ & Pred & Avg & Std & $\mathrm{SR}$ \\
\hline $\operatorname{Low}(\mathrm{L})$ & -0.45 & 0.18 & 5.60 & 0.11 & -0.38 & -0.29 & 7.02 & -0.14 & -0.23 & -0.54 & 7.83 & -0.24 \\
\hline 2 & -0.16 & 0.49 & 4.93 & 0.35 & 0.16 & 0.41 & 5.89 & 0.24 & 0.21 & 0.36 & 6.08 & 0.20 \\
\hline 3 & 0.02 & 0.59 & 4.75 & 0.43 & 0.44 & 0.51 & 5.07 & 0.35 & 0.44 & 0.65 & 5.07 & 0.44 \\
\hline 4 & 0.17 & 0.63 & 4.68 & 0.46 & 0.64 & 0.70 & 4.56 & 0.53 & 0.59 & 0.73 & 4.53 & 0.56 \\
\hline 5 & 0.34 & 0.57 & 4.70 & 0.42 & 0.80 & 0.77 & 4.37 & 0.61 & 0.72 & 0.81 & 4.38 & 0.64 \\
\hline 6 & 0.46 & 0.77 & 4.48 & 0.59 & 0.95 & 0.78 & 4.39 & 0.62 & 0.84 & 0.84 & 4.51 & 0.65 \\
\hline 7 & 0.59 & 0.52 & 4.73 & 0.38 & 1.11 & 0.81 & 4.40 & 0.64 & 0.97 & 0.95 & 4.61 & 0.71 \\
\hline 8 & 0.72 & 0.72 & 4.92 & 0.51 & 1.31 & 0.75 & 4.86 & 0.54 & 1.13 & 0.93 & 5.09 & 0.63 \\
\hline 9 & 0.88 & 0.99 & 5.19 & 0.66 & 1.58 & 0.96 & 5.22 & 0.64 & 1.37 & 1.04 & 5.69 & 0.63 \\
\hline $\operatorname{High}(\mathrm{H})$ & 1.11 & 1.17 & 5.88 & 0.69 & 2.19 & 1.52 & 6.79 & 0.77 & 1.99 & 1.38 & 6.98 & 0.69 \\
\hline \multirow[t]{3}{*}{ H-L } & 1.56 & 0.99 & 4.22 & 0.81 & 2.57 & 1.81 & 5.34 & 1.17 & 2.22 & 1.92 & 5.75 & 1.16 \\
\hline & \multicolumn{4}{|c|}{ NN3 } & \multicolumn{4}{|c|}{ NN4 } & \multicolumn{4}{|c|}{ NN5 } \\
\hline & Pred & Avg & Std & $\mathrm{SR}$ & Pred & Avg & Std & $\mathrm{SR}$ & Pred & Avg & Std & $\mathrm{SR}$ \\
\hline Low $(\mathrm{L})$ & -0.03 & -0.43 & 7.73 & -0.19 & -0.12 & -0.52 & 7.69 & -0.23 & -0.23 & -0.51 & 7.69 & -0.23 \\
\hline 2 & 0.34 & 0.30 & 6.38 & 0.16 & 0.30 & 0.33 & 6.16 & 0.19 & 0.23 & 0.31 & 6.10 & 0.17 \\
\hline 3 & 0.51 & 0.57 & 5.27 & 0.37 & 0.50 & 0.42 & 5.18 & 0.28 & 0.45 & 0.54 & 5.02 & 0.37 \\
\hline 4 & 0.63 & 0.66 & 4.69 & 0.49 & 0.62 & 0.60 & 4.51 & 0.46 & 0.60 & 0.67 & 4.47 & 0.52 \\
\hline 5 & 0.71 & 0.69 & 4.41 & 0.55 & 0.72 & 0.69 & 4.26 & 0.56 & 0.73 & 0.77 & 4.32 & 0.62 \\
\hline 6 & 0.79 & 0.76 & 4.46 & 0.59 & 0.81 & 0.84 & 4.46 & 0.65 & 0.85 & 0.86 & 4.35 & 0.68 \\
\hline 7 & 0.88 & 0.99 & 4.77 & 0.72 & 0.90 & 0.93 & 4.56 & 0.70 & 0.96 & 0.88 & 4.76 & 0.64 \\
\hline 8 & 1.00 & 1.09 & 5.47 & 0.69 & 1.03 & 1.08 & 5.13 & 0.73 & 1.11 & 0.94 & 5.17 & 0.63 \\
\hline 9 & 1.21 & 1.25 & 5.94 & 0.73 & 1.23 & 1.26 & 5.93 & 0.74 & 1.34 & 1.02 & 6.02 & 0.58 \\
\hline $\operatorname{High}(\mathrm{H})$ & 1.83 & 1.69 & 7.29 & 0.80 & 1.89 & 1.75 & 7.51 & 0.81 & 1.99 & 1.46 & 7.40 & 0.68 \\
\hline H-L & 1.86 & 2.12 & 6.13 & 1.20 & 2.01 & 2.26 & 5.80 & 1.35 & 2.22 & 1.97 & 5.93 & 1.15 \\
\hline
\end{tabular}

Note: In this table, we report the performance of prediction-sorted portfolios over the 30-year out-of-sample testing period. All stocks are sorted into deciles based on their predicted returns for the next month. Column "Pred", "Avg", "Std", and "SR" provide the predicted monthly returns for each decile, the average realized monthly returns, their standard deviations, and Sharpe ratios, respectively. All portfolios are value weighted. 
Table 8: Drawdowns, Turnover, and Risk-adjusted Performance of Machine Learning Portfolios

\begin{tabular}{|c|c|c|c|c|c|c|c|c|c|c|c|c|}
\hline & $\begin{array}{c}\text { OLS-3 } \\
+\mathrm{H}\end{array}$ & PLS & PCR & $\begin{array}{c}\text { ENet } \\
+\mathrm{H}\end{array}$ & $\begin{array}{c}\text { GLM } \\
+\mathrm{H}\end{array}$ & $\mathrm{RF}$ & $\begin{array}{c}\text { GBRT } \\
+\mathrm{H}\end{array}$ & NN1 & $\mathrm{NN} 2$ & NN3 & NN4 & NN5 \\
\hline & \multicolumn{12}{|c|}{ Drawdowns and Turnover (Value Weighted) } \\
\hline $\operatorname{Max} \mathrm{DD}(\%)$ & 69.60 & 41.13 & 42.17 & 60.71 & 37.09 & 52.27 & 48.75 & 61.60 & 55.29 & 30.84 & 51.78 & 57.52 \\
\hline Max 1M Loss(\%) & 24.72 & 27.40 & 18.38 & 27.40 & 15.61 & 26.21 & 21.83 & 18.59 & 37.02 & 30.84 & 33.03 & 38.95 \\
\hline \multirow[t]{2}{*}{ Turnover $(\%)$} & 58.20 & 110.87 & 125.86 & 151.59 & 145.26 & 133.87 & 143.53 & 121.02 & 122.46 & 123.50 & 126.81 & 125.37 \\
\hline & \multicolumn{12}{|c|}{ Drawdowns and Turnover (Equally Weighted) } \\
\hline $\operatorname{Max} \mathrm{DD}(\%)$ & 84.74 & 32.35 & 31.39 & 33.70 & 21.01 & 46.42 & 37.19 & 18.25 & 25.81 & 17.34 & 14.72 & 21.78 \\
\hline Max 1M Loss(\%) & 37.94 & 32.35 & 22.33 & 32.35 & 15.74 & 34.63 & 22.34 & 12.79 & 25.81 & 12.50 & 9.01 & 21.78 \\
\hline \multirow[t]{2}{*}{ Turnover $(\%)$} & 57.24 & 104.47 & 118.07 & 142.78 & 137.97 & 120.29 & 134.24 & 112.35 & 112.43 & 113.76 & 114.17 & 114.34 \\
\hline & \multicolumn{12}{|c|}{ Risk-adjusted Performance (Value Weighted) } \\
\hline Mean Ret. & 0.94 & 1.02 & 1.22 & 0.60 & 1.06 & 1.62 & 0.99 & 1.81 & 1.92 & 1.97 & 2.26 & 2.12 \\
\hline FF5+Mom $\alpha$ & 0.39 & 0.24 & 0.62 & -0.23 & 0.38 & 1.20 & 0.66 & 1.20 & 1.33 & 1.52 & 1.76 & 1.43 \\
\hline t-stats & 2.76 & 1.09 & 2.89 & -0.89 & 1.68 & 3.95 & 3.11 & 4.68 & 4.74 & 4.92 & 6.00 & 4.71 \\
\hline$R^{2}$ & 78.60 & 34.95 & 39.11 & 28.04 & 30.78 & 13.43 & 20.68 & 27.67 & 25.81 & 20.84 & 20.47 & 18.23 \\
\hline \multirow[t]{2}{*}{ IR } & 0.54 & 0.21 & 0.57 & -0.17 & 0.33 & 0.77 & 0.61 & 0.92 & 0.93 & 0.96 & 1.18 & 0.92 \\
\hline & \multicolumn{12}{|c|}{ Risk-adjusted Performance (Equally Weighted) } \\
\hline Mean Ret. & 1.34 & 2.08 & 2.45 & 2.11 & 2.31 & 2.38 & 2.14 & 2.91 & 3.31 & 3.27 & 3.33 & 3.09 \\
\hline FF5+Mom $\alpha$ & 0.83 & 1.40 & 1.95 & 1.32 & 1.79 & 1.88 & 1.87 & 2.60 & 3.07 & 3.02 & 3.08 & 2.78 \\
\hline$t(\alpha)$ & 6.64 & 5.90 & 9.92 & 4.77 & 8.09 & 6.66 & 8.19 & 10.51 & 11.66 & 11.70 & 12.28 & 10.68 \\
\hline$R^{2}$ & 84.26 & 26.27 & 40.50 & 20.89 & 21.25 & 19.91 & 11.19 & 13.98 & 10.60 & 9.63 & 11.57 & 14.54 \\
\hline IR & 1.30 & 1.15 & 1.94 & 0.93 & 1.58 & 1.30 & 1.60 & 2.06 & 2.28 & 2.29 & 2.40 & 2.09 \\
\hline
\end{tabular}

Note: The top two panels report a value-weight and equal-weight portfolio maximum drawdown ("Max DD"), most extreme negative monthly return ("Max 1M Loss"), and average monthly percentage change in holdings ("Turnover"). The bottom panel report average monthly returns in percent as well as alphas, information ratios (IR), and $R^{2}$ with respect to the Fama-French five-factor model augmented to include the momentum factor.

OLS-3, but are nonetheless handily outperformed by tree-based models and neural networks.

The top panel of Table 8 reports drawdowns, portfolio turnover, and risk-adjusted performance of 10-1 spread portfolios from each machine learning model, for both value and equally weighted strategies. We define maximum drawdown of a strategy as

$$
\operatorname{MaxDD}=\max _{0 \leq t_{1} \leq t_{2} \leq T}\left(Y_{t_{1}}-Y_{t_{2}}\right)
$$

where $Y_{t}$ is the cumulative log return from date 0 through $t$.

Not only do neural network portfolios have higher Sharpe ratios than alternatives, they also have comparatively small drawdowns, particularly for equal-weight portfolios. The maximum drawdown experienced for the NN4 strategy is $51.8 \%$ and $14.7 \%$ for value and equal weights, respectively. In contrast, the maximum drawdown for OLS-3 are $69.6 \%$ and $84.7 \%$, respectively. Equal-weight neural network strategies also experience the most mild one-month losses. For example, for NN4, the worst one-month performance is a $-9.01 \%$ return, which is the least extreme monthly loss among all models with either weighting scheme. 
We define the strategy's average monthly turnover as

$$
\text { Turnover }=\frac{1}{T} \sum_{t=1}^{T}\left(\sum_{i}\left|w_{i, t+1}-\frac{w_{i, t}\left(1+r_{i, t+1}\right)}{\sum_{j} w_{j, t}\left(1+r_{j, t+1}\right)}\right|\right),
$$

where $w_{i, t}$ is the weight of stock $i$ in the portfolio at time $t$. For NN1 through NN5, turnover is consistently between $110 \%$ and $130 \%$ per month, and turnover for tree-based methods and penalized regression is slightly higher. As a frame of reference, the monthly turnover of a short-term reversal decile spread is $172.6 \%$ per month while for a size decile spread it is $22.9 \%$. Given the large role of price trend predictors selected by all machine learning approaches, it is perhaps unsurprising that their outperformance is accomplished with comparatively high portfolio turnover.

The bottom panel of Table 8 reports risk-adjusted performance of machine learning portfolios based on factor pricing models. In a linear factor model, the tangency portfolio of the factors themselves represents the maximum Sharpe ratio portfolio in the economy. Any portfolio with a higher Sharpe ratio than the factor tangency portfolio possesses alpha with respect to the model. From prior work, the out-of-sample factor tangency portfolios of the Fama-French three and fivefactor models have Sharpe ratios of roughly 0.8 and 1.3, respectively (Kelly et al., 2019). It is unsurprising then that portfolios formed on the basis of machine learning forecasts earn large and significant alphas versus these models. A six-factor model (that appends a momentum factor to the Fama-French five-factor model) explains as much as $40 \%$ of the average return for strategies based on linear models, but explains only about $10 \%$ to $30 \%$ of variation in neural network-based portfolios. As a result, neural networks have information ratios ranging from 0.9 to 2.4 depending on the number of layers and the portfolio weighting scheme. Test statistics for the associated alphas are highly significant for both tree models and all neural network models.

The results of Table 8 are visually summarized in Figure 9, which reports cumulative performance for the long and short sides for select strategies, along with the cumulative market excess return as a benchmark. NN4 dominates the other models by a large margin in both directions. Interestingly, the short side of all portfolios is essentially flat in the post-2000 sample. ${ }^{39}$

Finally, we consider two meta-strategies that combine forecasts of all machine learning portfolios. The first is a simple equally weighted average of decile long-short portfolios from our 11 machine learning methods. This achieves a stock-level predictive $R^{2}$ of $0.43 \%$ per month and an equally weighted decile spread Sharpe ratio of 2.49 , both of which are higher than any single method on its own. The value weighted decile spread Sharpe ratio is 1.33 , which is slightly lower than that for the NN4 model.

The second meta-strategy rotates among machine learning methods by selecting the best machine learning model for each one-year test sample based on the predictive $R^{2}$ during the corresponding validation sample. Over our 30-year out-of-sample period, this method selects NN3 11 times, NN1 seven times, GBRT six times, NN2 five times, and NN4 one time. This method delivers the highest overall panel $R^{2}(0.45 \%)$, but underperforms the standalone NN4 model in terms of decile spread Sharpe

\footnotetext{
${ }^{39}$ The corresponding plot of cumulative returns for equal-weight strategies is shown in appendix Figure A.7.
} 
Figure 9: Cumulative Return of Machine Learning Portfolios

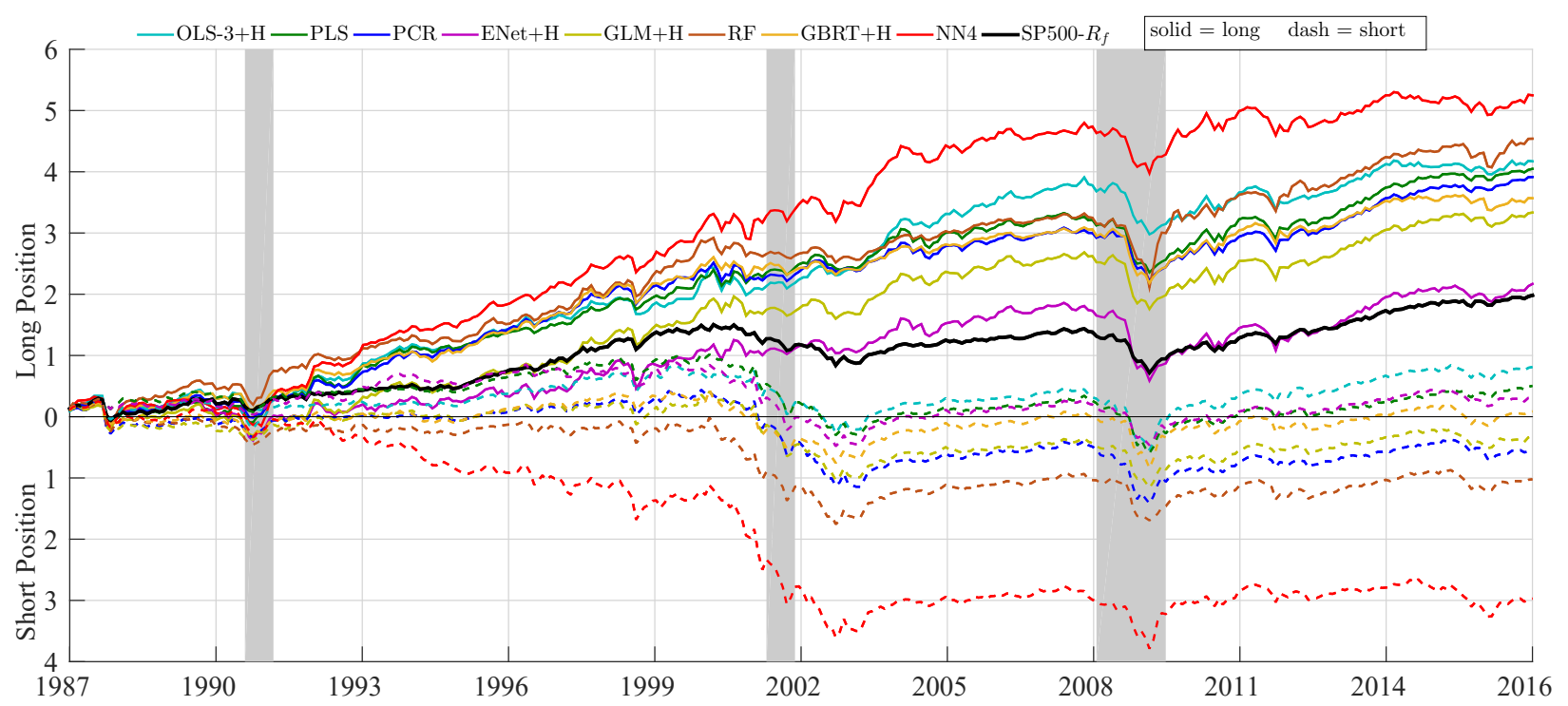

Note: Cumulative log returns of portfolios sorted on out-of-sample machine learning return forecasts. The solid and dash lines represent long (top decile) and short (bottom decile) positions, respectively. The shaded periods show NBER recession dates. All portfolios are value weighted.

ratio (2.42 and 1.23 with equal and value weights, respectively). Interestingly, the meta-strategy's predictive $R^{2}$ gain is statistically insignificant relative to the best standalone neural network models.

\section{Conclusion}

Using the empirical context of return prediction as a proving ground, we perform a comparative analysis of methods in the machine learning repertoire. At the highest level, our findings demonstrate that machine learning methods can help improve our empirical understanding of asset prices. Neural networks and, to a lesser extent, regression trees, are the best performing methods. We track down the source of their predictive advantage to accommodation of nonlinear interactions that are missed by other methods. We also find that "shallow" learning outperforms "deep" learning, which differs from the typical conclusion in other fields such as computer vision or bioinformatics, and is likely due to the comparative dearth of data and low signal-to-noise ratio in asset pricing problems. Machine learning methods are most valuable for forecasting larger and more liquid stock returns and portfolios. Lastly, we find that all methods agree on a fairly small set of dominant predictive signals, the most powerful predictors being associated with price trends including return reversal and momentum. The next most powerful predictors are measures of stock liquidity, stock volatility, and valuation ratios.

The overall success of machine learning algorithms for return prediction brings promise for both economic modeling and for practical aspects of portfolio choice. With better measurement through machine learning, risk premia are less shrouded in approximation and estimation error, thus the challenge of identifying reliable economic mechanisms behind asset pricing phenomena becomes less 
steep. Finally, our findings help justify the growing role of machine learning throughout the architecture of the burgeoning fintech industry.

\section{References}

Bach, Francis R., 2008, Consistency of the group lasso and multiple kernel learning, Journal of Machine Learning Research 9, 1179-1225.

Bai, Jushan, and Serena Ng, 2002, Determining the number of factors in approximate factor models, Econometrica 70, 191-221.

Bai, Jushan, and Serena Ng, 2013, Principal components estimation and identification of static factors, Journal of Econometrics 176, 18-29.

Bai, Zhidong, 1999, Methodologies in spectral analysis of large dimensional random matrices: A review, Statistica Sinica 9, 611-677.

Biau, Gérard, 2012, Analysis of a random forests model, Journal of Machine Learning Research 13, $1063-1095$.

Bickel, Peter J, Ya'acov Ritov, and Alexandre B Tsybakov, 2009, Simultaneous analysis of Lasso and Dantzig selector, Annals of Statistics 37, 1705-1732.

Bishop, Christopher M., 1995, Neural Networks for Pattern Recognition (Oxford University Press).

Box, G. E. P., 1953, Non-normality and tests on variances, Biometrika 40, 318-335.

Breiman, Leo, 2001, Random forests, Machine learning 45, 5-32.

Breiman, Leo, Jerome Friedman, Charles J Stone, and Richard A Olshen, 1984, Classification and regression trees (CRC press).

Bühlmann, Peter, and Torsten Hothorn, 2007, Boosting Algorithms: Regularization, Prediction and Model Fitting, Statistical Science 22, 477-505.

Bühlmann, Peter, and Bin Yu, 2003, Boosting with the 12 loss, Journal of the American Statistical Association 98, 324-339.

Butaru, Florentin, Qingqing Chen, Brian Clark, Sanmay Das, Andrew W Lo, and Akhtar Siddique, 2016, Risk and risk management in the credit card industry, Journal of Banking 8 Finance 72, $218-239$.

Campbell, John Y., and S. B. Thompson, 2008, Predicting excess stock returns out of sample: Can anyting beat the historical average?, Review of Financial Studies 21, 1509-1531.

Campbell, John Y, and Samuel B Thompson, 2007, Predicting excess stock returns out of sample: Can anything beat the historical average?, The Review of Financial Studies 21, 1509-1531.

Chen, Tianqi, and Carlos Guestrin, 2016, Xgboost: A scalable tree boosting system, in Proceedings of the 22Nd ACM SIGKDD International Conference on Knowledge Discovery and Data Mining, KDD '16, 785-794 (ACM, New York, NY, USA). 
Chizat, Lénaïc, and Francis Bach, 2018, On the global convergence of gradient descent for overparameterized models using optimal transport, in Proceedings of the 32Nd International Conference on Neural Information Processing Systems, NIPS'18, 3040-3050 (Curran Associates Inc., USA).

Cochrane, John H, 2007, The dog that did not bark: A defense of return predictability, The Review of Financial Studies 21, 1533-1575.

Cybenko, George, 1989, Approximation by superpositions of a sigmoidal function, Mathematics of control, signals and systems 2, 303-314.

Daubechies, I, M Defrise, and C De Mol, 2004, An iterative thresholding algorithm for linear inverse problems with a sparsity constraint, Communications on Pure and Applied Mathematics 57, 14131457.

de Jong, Sijmen, 1993, Simpls: An alternative approach to partial least squares regression, Chemometrics and Intelligent Laboratory Systems 18, 251 - 263.

Diebold, Francis X., 2015, Comparing predictive accuracy, twenty years later: A personal perspective on the use and abuse of diebold-mariano tests, Journal of Business 86 Economic Statistics 33, 1-9.

Diebold, Francis X., and Roberto S. Mariano, 1995, Comparing predictive accuracy, Journal of Business \& Economic Statistics 13, 134-144.

Dietterich, Thomas G, 2000, Ensemble methods in machine learning, in International workshop on multiple classifier systems, 1-15, Springer.

Dimopoulos, Yannis, Paul Bourret, and Sovan Lek, 1995, Use of some sensitivity criteria for choosing networks with good generalization ability, Neural Processing Letters 2, 1-4.

Eldan, Ronen, and Ohad Shamir, 2016, The power of depth for feedforward neural networks, in Vitaly Feldman, Alexander Rakhlin, and Ohad Shamir, eds., 29th Annual Conference on Learning Theory, volume 49 of Proceedings of Machine Learning Research, 907-940 (PMLR, Columbia University, New York, New York, USA).

Fama, Eugene F, and Kenneth R French, 1993, Common risk factors in the returns on stocks and bonds, Journal of financial economics 33, 3-56.

Fama, Eugene F, and Kenneth R French, 2008, Dissecting anomalies, The Journal of Finance 63, $1653-1678$.

Fama, Eugene F., and Kenneth R. French, 2015, A five-factor asset pricing model, Journal of Financial Economics 116, 1-22.

Fan, Jianqing, Quefeng Li, and Yuyan Wang, 2017, Estimation of high dimensional mean regression in the absence of symmetry and light tail assumptions, Journal of the Royal Statistical Society, B $79,247-265$.

Fan, Jianqing, Cong Ma, and Yiqiao Zhong, 2019, A selective overview of deep learning, Technical report, Princeton University.

Feng, Guanhao, Stefano Giglio, and Dacheng Xiu, 2019, Taming the factor zoo: A test of new factors, Journal of Finance, forthcoming . 
Freund, Yoav, 1995, Boosting a weak learning algorithm by majority, Information and Computation 121, 256-285.

Freyberger, Joachim, Andreas Neuhierl, and Michael Weber, 2019, Dissecting characteristics nonparametrically, Review of Financial Studies, forthcoming .

Friedman, Jerome, Trevor Hastie, Holger Höfling, Robert Tibshirani, et al., 2007, Pathwise coordinate optimization, The Annals of Applied Statistics 1, 302-332.

Friedman, Jerome, Trevor Hastie, and Robert Tibshirani, 2000a, Additive logistic regression: A statistical view of boosting, Annals of Statistics 28, 337-374.

Friedman, Jerome, Trevor Hastie, and Robert Tibshirani, 2000b, Additive logistic regression: a statistical view of boosting (with discussion and a rejoinder by the authors), Annals of Statistics $28,337-407$.

Friedman, Jerome H, 2001, Greedy function approximation: a gradient boosting machine, Annals of statistics $1189-1232$.

Giglio, Stefano W, and Dacheng Xiu, 2016, Asset pricing with omitted factors, Technical report, University of Chicago.

Glorot, Xavier, Antoine Bordes, and Yoshua Bengio, 2011, Deep sparse rectifier neural networks., in Aistats, volume 15, 315-323.

Goodfellow, Ian, Yoshua Bengio, and Aaron Courville, 2016, Deep Learning (MIT Press), http: //www. deeplearningbook.org.

Green, Jeremiah, John RM Hand, and X Frank Zhang, 2013, The supraview of return predictive signals, Review of Accounting Studies 18, 692-730.

Green, Jeremiah, John RM Hand, and X Frank Zhang, 2017, The characteristics that provide independent information about average us monthly stock returns, The Review of Financial Studies $30,4389-4436$.

Gu, Shihao, Bryan T Kelly, and Dacheng Xiu, 2019, Autoencoder asset pricing models, Available at SSRN .

Hansen, Lars Kai, and Peter Salamon, 1990, Neural network ensembles, IEEE transactions on pattern analysis and machine intelligence 12, 993-1001.

Harvey, Campbell R., and Wayne E. Ferson, 1999, Conditioning variables and the cross-section of stock returns, Journal of Finance 54, 1325-1360.

Harvey, Campbell R, and Yan Liu, 2016, Lucky factors, Technical report, Duke University.

Harvey, Campbell R, Yan Liu, and Heqing Zhu, 2016, ... and the cross-section of expected returns, Review of Financial Studies 29, 5-68.

Hastie, Trevor, Robert Tibshirani, and Jerome Friedman, 2009, The Elements of Statistical Learning (Springer).

He, Kaiming, Xiangyu Zhang, Shaoqing Ren, and Jian Sun, 2016a, Deep residual learning for image recognition, in 2016 IEEE Conference on Computer Vision and Pattern Recognition, CVPR 2016, Las Vegas, NV, USA, June 27-30, 2016, 770-778. 
He, Kaiming, Xiangyu Zhang, Shaoqing Ren, and Jian Sun, 2016b, Deep residual learning for image recognition, in The IEEE Conference on Computer Vision and Pattern Recognition (CVPR).

Heaton, JB, NG Polson, and JH Witte, 2016, Deep learning in finance, arXiv preprint arXiv:1602.06561.

Hinton, Geoffrey E., Simon Osindero, and Yee-Whye Teh, 2006, A fast learning algorithm for deep belief nets, Neural Comput. 18, 1527-1554.

Hornik, Kurt, Maxwell Stinchcombe, and Halbert White, 1989, Multilayer feedforward networks are universal approximators, Neural networks 2, 359-366.

Huber, P. J., 1964, Robust estimation of a location parameter, Annals of Mathematical Statistics 35, 73-101.

Hutchinson, James M, Andrew W Lo, and Tomaso Poggio, 1994, A nonparametric approach to pricing and hedging derivative securities via learning networks, The Journal of Finance 49, 851889.

Ioffe, Sergey, and Christian Szegedy, 2015, Batch Normalization: Accelerating Deep Network Training by Reducing Internal Covariate Shift, International Conference on Machine Learning 448-456.

Jaggi, Martin, 2013, An equivalence between the lasso and support vector machines, Regularization, optimization, kernels, and support vector machines 1-26.

Jarrett, Kevin, Koray Kavukcuoglu, Yann Lecun, et al., 2009, What is the best multi-stage architecture for object recognition?, in 2009 IEEE 12th International Conference on Computer Vision, 2146-2153, IEEE.

Johnstone, Iain M., 2001, On the distribution of the largest eigenvalue in principal components analysis, Annals of Statistics 29, 295-327.

Johnstone, Iain M., and Arthur Yu Lu, 2009, On consistency and sparsity for principal components analysis in high dimensions, Journal of the American Statistical Association 104, 682-693.

Kelly, Bryan, and Seth Pruitt, 2013, Market expectations in the cross-section of present values, The Journal of Finance 68, 1721-1756.

Kelly, Bryan, and Seth Pruitt, 2015, The three-pass regression filter: A new approach to forecasting using many predictors, Journal of Econometrics 186, 294-316.

Kelly, Bryan, Seth Pruitt, and Yinan Su, 2019, Some characteristics are risk exposures, and the rest are irrelevant, Journal of Financial Economics, forthcoming .

Khandani, Amir E, Adlar J Kim, and Andrew W Lo, 2010, Consumer credit-risk models via machinelearning algorithms, Journal of Banking 83 Finance 34, 2767-2787.

Kingma, Diederik, and Jimmy Ba, 2014, Adam: A method for stochastic optimization, arXiv preprint arXiv:1412.6980 .

Knight, Keith, and Wenjiang Fu, 2000, Asymptotics for lasso-type estimators, Annals of Statistics $28,1356-1378$.

Koijen, Ralph, and Stijn Van Nieuwerburgh, 2011, Predictability of returns and cash flows, Annual Review of Financial Economics 3, 467-491. 
Kozak, Serhiy, 2019, Kernel trick for the cross section, Available at SSRN 3307895 .

Kozak, Serhiy, Stefan Nagel, and Shrihari Santosh, 2019, Shrinking the cross section, Journal of Financial Economics, forthcoming .

Lewellen, Jonathan, 2015, The cross-section of expected stock returns, Critical Finance Review 4, $1-44$.

Lin, Henry W., Max Tegmark, and David Rolnick, 2017, Why does deep and cheap learning work so well?, Journal of Statistical Physics 168, 1223-1247.

Lo, Andrew W, and A Craig MacKinlay, 1990, Data-snooping biases in tests of financial asset pricing models, Review of financial studies 3, 431-467.

Lounici, Karim, Massimiliano Pontil, Sara van de Geer, and Alexandre B. Tsybakov, 2011, Oracle inequalities and optimal inference under group sparsity, Annals of Statistics 39, 2164-2204.

Lugosi, Gábor, and Nicolas Vayatis, 2004, On the bayes-risk consistency of regularized boosting methods, Annals of Statistics 32, 30-55.

Masters, Timothy, 1993, Practical Neural Network Recipes in C++ (Academic Press).

Mei, Song, Theodor Misiakiewicz, and Andrea Montanari, 2019, Mean-field theory of two-layers neural networks: dimension-free bounds and kernel limit, in Conference on Learning Theory (COLT).

Mei, Song, Andrea Montanari, and Phan-Minh Nguyen, 2018, A mean field view of the landscape of two-layer neural networks, Proceedings of the National Academy of Sciences 115, E7665-E7671.

Meinshausen, Nicolai, and Bin Yu, 2009, Lasso-type recovery of sparse representations for highdimensional data, Annals of Statistics 37, 246-270.

Mentch, Lucas, and Giles Hooker, 2016, Quantifying uncertainty in random forests via confidence intervals and hypothesis tests, Journal of Machine Learning Research 17, 1-41.

Mol, Christine De, Ernesto De Vito, and Lorenzo Rosasco, 2009, Elastic-net regularization in learning theory, Journal of Complexity 25, $201-230$.

Moritz, Benjamin, and Tom Zimmermann, 2016, Tree-based conditional portfolio sorts: The relation between past and future stock returns, Available at SSRN 2740751 .

Nair, Vinod, and Geoffrey E Hinton, 2010, Rectified linear units improve restricted boltzmann machines, in Proceedings of the 27th International Conference on Machine Learning (ICML-10), $807-814$.

Nesterov, Yurii, 1983, A method of solving a convex programming problem with convergence rate $o\left(1 / k^{2}\right)$, Soviet Mathematics Doklady 27, 372-376.

Parikh, Neal, and Stephen Boyd, 2013, Proximal algorithms, Foundations and Trends in Optimization $1,123-231$.

Paul, Debashis, 2007, Asymptotics of sample eigenstructure for a large dimensional spiked covariance model, Statistical Sinica 17, 1617-1642.

Polson, Nicholas G., James Scott, and Brandon T. Willard, 2015, Proximal algorithms in statistics and machine learning, Statistical Science 30, 559-581. 
Rapach, David, and Guofu Zhou, 2013, Forecasting stock returns. vol. 2 of handbook of economic forecasting, 328383 .

Rapach, David E, Jack K Strauss, and Guofu Zhou, 2013, International stock return predictability: what is the role of the united states?, The Journal of Finance 68, 1633-1662.

Ravikumar, Pradeep, John Lafferty, Han Liu, and Larry Wasserman, 2009, Sparse additive models, Journal of the Royal Statistical Society. Series B (Statistical Methodology) 71, 1009-1030.

Rolnick, David, and Max Tegmark, 2018, The power of deeper networks for expressing natural functions, in ICLR.

Rosenberg, Barr, 1974, Extra-market components of covariance in security returns, Journal of Financial and Quantitative Analysis 9, 263-274.

Schapire, Robert E., 1990, The strength of weak learnability, Machine Learning 5, 197-227.

Scornet, Erwan, Gérard Biau, and Jean-Philippe Vert, 2015, Consistency of random forests, Annals of Statistics 43, 1716-1741.

Sirignano, Justin, Apaar Sadhwani, and Kay Giesecke, 2016, Deep learning for mortgage risk, Available at SSRN 2799443 .

Stock, James H., and Mark W. Watson, 2002, Forecasting using principal components from a large number of predictors, Journal of American Statistical Association 97, 1167-1179.

Tibshirani, Robert, 2011, Regression shrinkage and selection via the lasso: a retrospective, Journal of the Royal Statistical Society: Series B (Statistical Methodology) 73, 273-282.

Tukey, J. W., 1960, A survey of sampling from contaminated distributions, in I. Olkin, S. G. Ghurye, W. Hoeffding, W. G. Madow, and H. B. Mann, eds., Contributions to probability and statistics: Essays in Honor of Harold Hotelling (Stanford University Press).

Wager, Stefan, and Susan Athey, 2018, Estimation and inference of heterogeneous treatment effects using random forests, Journal of the American Statistical Association 113, 1228-1242.

Wager, Stefan, Trevor Hastie, and Bradley Efron, 2014, Confidence intervals for random forests: The jackknife and the infinitesimal jackknife, Journal of Machine Learning Research 15, 1625-1651.

Wainwright, Martin J., 2009, Sharp thresholds for high-dimensional and noisy sparsity recovery using $l_{1}$-constrained quadratic programming (lasso), IEEE Transactions on Information Theory $55,2183-2202$.

Wang, Weichen, and Jianqing Fan, 2017, Asymptotics of empirical eigenstructure for high dimensional spiked covariance, Annals of Statistics 45, 1342-1374.

Welch, Ivo, and Amit Goyal, 2008, A Comprehensive Look at The Empirical Performance of Equity Premium Prediction, Review of Financial Studies 21, 1455-1508.

West, Kenneth D., 2006, Forecast evaluation, in Graham Elliott, Cliver Granger, and Allan Timmermann, eds., Handbook of Economic Forecasting, volume 1, 99-134 (Elsevier).

White, Halbert, 1980, Using least squares to approximate unknown regression functions, International Economic Review 149-170. 
Wilson, D. Randall, and Tony R. Martinez, 2003, The general inefficiency of batch training for gradient descent learning, Neural networks 16, 1429-1451.

Yao, Jingtao, Yili Li, and Chew Lim Tan, 2000, Option price forecasting using neural networks, Omega 28, 455-466.

Zhang, Cun-Hui, and Jian Huang, 2008, The sparsity and bias of the lasso selection in highdimensional linear regression, Annals of Statistics 36, 1567-1594.

Zhang, Tong, and Bin Yu, 2005, Boosting with early stopping: Convergence and consistency, Annals of Statistics 33, 1538-1579.

Zou, Hui, and Trevor Hastie, 2005, Regularization and variable selection via the elastic net, Journal of the Royal Statistical Society. Series B (Statistical Methodology) 67, 301-320.

Zou, Hui, and Hao Helen Zhang, 2009, On the adaptive elastic-net with a diverging number of parameters, Annals of Statistics 37, 1733-1751. 


\section{Internet Appendix}

\section{A Monte Carlo Simulations}

To demonstrate the finite sample performance of all machine learning procedures, we simulate a (latent) 3-factor model for excess returns $r_{t+1}$, for $t=1,2, \ldots, T$ :

$$
r_{i, t+1}=g^{\star}\left(z_{i, t}\right)+e_{i, t+1}, \quad e_{i, t+1}=\beta_{i, t} v_{t+1}+\varepsilon_{i, t+1}, \quad z_{i, t}=\left(1, x_{t}\right)^{\prime} \otimes c_{i, t}, \quad \beta_{i, t}=\left(c_{i 1, t}, c_{i 2, t}, c_{i 3, t}\right),
$$

where $c_{t}$ is an $N \times P_{c}$ matrix of characteristics, $v_{t+1}$ is a $3 \times 1$ vector of factors, $x_{t}$ is a univariate time series, and $\varepsilon_{t+1}$ is a $N \times 1$ vector of idiosyncratic errors. We choose $v_{t+1} \sim \mathcal{N}\left(0,0.05^{2} \times \mathbb{I}_{3}\right)$, and $\varepsilon_{i, t+1} \sim t_{5}\left(0,0.05^{2}\right)$, in which their variances are calibrated so that the average time series $R^{2}$ is $40 \%$ and the average annualized volatility is $30 \%$.

We simulate the panel of characteristics for each $1 \leq i \leq N$ and each $1 \leq j \leq P_{c}$ from the following model:

$$
c_{i j, t}=\frac{2}{N+1} \operatorname{CSrank}\left(\bar{c}_{i j, t}\right)-1, \quad \bar{c}_{i j, t}=\rho_{j} \bar{c}_{i j, t-1}+\epsilon_{i j, t}
$$

where $\rho_{j} \sim \mathcal{U}[0.9,1], \epsilon_{i j, t} \sim \mathcal{N}\left(0,1-\rho_{j}^{2}\right)$ and CSrank is Cross-Section rank function, so that the characteristics feature some degree of persistence over time, yet is cross-sectionally normalized to be within $[-1,1]$. This matches our data cleaning procedure in the empirical study.

In addition, we simulate the time series $x_{t}$ from the following model:

$$
x_{t}=\rho x_{t-1}+u_{t}
$$

where $u_{t} \sim \mathcal{N}\left(0,1-\rho^{2}\right)$, and $\rho=0.95$ so that $x_{t}$ is highly persistent.

We consider two cases of $g^{\star}(\cdot)$ functions:

(a) $g^{\star}\left(z_{i, t}\right)=\left(c_{i 1, t}, c_{i 2, t}, c_{i 3, t} \times x_{t}\right) \theta_{0}, \quad$ where $\theta_{0}=(0.02,0.02,0.02)^{\prime}$;

(b) $g^{\star}\left(z_{i, t}\right)=\left(c_{i 1, t}^{2}, c_{i 1, t} \times c_{i 2, t}, \operatorname{sgn}\left(c_{i 3, t} \times x_{t}\right)\right) \theta_{0}, \quad$ where $\theta_{0}=(0.04,0.03,0.012)^{\prime}$.

In both cases, $g^{\star}(\cdot)$ only depends on 3 covariates, so there are only 3 non-zero entries in $\theta$, denoted as $\theta_{0}$. Case $(a)$ is simple and sparse linear model. Case $(b)$ involves a nonlinear covariate $c_{i 1, t}^{2}$, a nonlinear and interaction term $c_{i 1, t} \times c_{i 2, t}$, and a discrete variable $\operatorname{sgn}\left(c_{i 3, t} \times x_{t}\right)$. We calibrate the values of $\theta_{0}$ such that the cross-sectional $R^{2}$ is $50 \%$, and the predictive $R^{2}$ is $5 \%$.

Throughout, we fix $N=200, T=180$, and $P_{x}=2$, while comparing the cases of $P_{c}=100$ and $P_{c}=50$, corresponding to $P=200$ and 100 , respectively, to demonstrate the effect of increasing dimensionality.

For each Monte Carlo sample, we divide the whole time series into 3 consecutive subsamples of equal length for training, validation, and testing, respectively. Specifically, we estimate each of the two models in the training sample, using PLS, PCR, Ridge, Lasso, Elastic Net (ENet), generalized 
Table A.1: Comparison of Predictive $R^{2}$ s for Machine Learning Algorithms in Simulations Model $(a)$ (b)

\begin{tabular}{|c|c|c|c|c|c|c|c|c|}
\hline \multirow{2}{*}{$\frac{\text { Parameter }}{R^{2}(\%)}$} & \multicolumn{2}{|c|}{$P_{c}=50$} & \multicolumn{2}{|c|}{$P_{c}=100$} & \multicolumn{2}{|c|}{$P_{c}=50$} & \multicolumn{2}{|c|}{$P_{c}=100$} \\
\hline & IS & OOS & IS & OOS & IS & OOS & IS & OOS \\
\hline OLS & 7.50 & 1.14 & 8.19 & -1.35 & 3.44 & -4.72 & 4.39 & -7.75 \\
\hline $\mathrm{OLS}+\mathrm{H}$ & 7.48 & 1.25 & 8.16 & -1.15 & 3.43 & -4.60 & 4.36 & -7.54 \\
\hline PCR & 2.69 & 0.90 & 1.70 & 0.43 & 0.65 & 0.02 & 0.41 & -0.01 \\
\hline PLS & 6.24 & 3.48 & 6.19 & 2.82 & 1.02 & -0.08 & 0.99 & -0.17 \\
\hline Lasso & 6.04 & 4.26 & 6.08 & 4.25 & 1.36 & 0.58 & 1.36 & 0.61 \\
\hline Lasso $+\mathrm{H}$ & 6.00 & 4.26 & 6.03 & 4.25 & 1.32 & 0.59 & 1.31 & 0.61 \\
\hline Ridge & 6.46 & 3.89 & 6.67 & 3.39 & 1.66 & 0.34 & 1.76 & 0.23 \\
\hline Ridge $+\mathrm{H}$ & 6.42 & 3.91 & 6.61 & 3.42 & 1.63 & 0.35 & 1.73 & 0.25 \\
\hline ENet & 6.04 & 4.26 & 6.08 & 4.25 & 1.35 & 0.58 & 1.35 & 0.61 \\
\hline ENet $+\mathrm{H}$ & 6.00 & 4.26 & 6.03 & 4.25 & 1.32 & 0.59 & 1.31 & 0.61 \\
\hline GLM & 5.91 & 4.11 & 5.94 & 4.08 & 3.38 & 1.22 & 3.31 & 1.17 \\
\hline $\mathrm{GLM}+\mathrm{H}$ & 5.85 & 4.12 & 5.88 & 4.09 & 3.32 & 1.24 & 3.24 & 1.20 \\
\hline $\mathrm{RF}$ & 8.34 & 3.35 & 8.23 & 3.30 & 8.05 & 3.07 & 8.22 & 3.02 \\
\hline GBRT & 7.08 & 3.35 & 7.02 & 3.33 & 6.51 & 2.76 & 6.42 & 2.84 \\
\hline $\mathrm{GBRT}+\mathrm{H}$ & 7.16 & 3.45 & 7.11 & 3.37 & 6.47 & 3.12 & 6.37 & 3.22 \\
\hline NN1 & 6.53 & 4.37 & 6.72 & 4.28 & 5.61 & 2.78 & 5.80 & 2.59 \\
\hline NN2 & 6.55 & 4.42 & 6.72 & 4.26 & 6.22 & 3.13 & 6.33 & 2.91 \\
\hline NN3 & 6.47 & 4.34 & 6.67 & 4.27 & 6.03 & 2.96 & 6.09 & 2.68 \\
\hline NN4 & 6.47 & 4.31 & 6.66 & 4.24 & 5.94 & 2.81 & 6.04 & 2.51 \\
\hline NN5 & 6.41 & 4.27 & 6.55 & 4.14 & 5.81 & 2.72 & 5.70 & 2.20 \\
\hline Oracle & 6.22 & 5.52 & 6.22 & 5.52 & 5.86 & 5.40 & 5.86 & 5.40 \\
\hline
\end{tabular}

Note: In this table, we report the average in-sample (IS) and out-of-sample (OOS) $R^{2}$ for models $(a)$ and $(b)$ using Ridge, Lasso, Elastic Net (ENet), generalized linear model with group lasso (GLM), random forest (RF), gradient boosted regression trees (GBRT), and five architectures of neural networks (NN1,..,NN5), respectively. "+H" indicates the use of Huber loss instead of the $l_{2}$ loss. "Oracle" stands for using the true covariates in a pooled-OLS regression. We fix $N=200, T=180$, and $P_{x}=2$, comparing $P_{c}=100$ with $P_{c}=50$. The number of Monte Carlo repetitions is 100 .

linear model with group lasso (GLM), random forest (RF), gradient boosted regression trees (GBRT), and the same five architectures of neural networks (NN1,..,NN5) we adopt for the empirical work, respectively, then choose tuning parameters for each method in the validation sample, and calculate the prediction errors in the testing sample. For benchmark, we also compare the pooled OLS with all covariates and that using the oracle model.

We report the average $R^{2}$ s both in-sample (IS) and out-of-sample (OOS) for each model and each method over 100 Monte Carlo repetitions in Table A.1. Both IS and OOS $R^{2}$ are relative to the estimator based on the IS average. For model (a), Lasso, ENet and NNs deliver the best and almost identical out-of-sample $R^{2}$. This is not surprising given that the true model is sparse and linear in the input covariates. The advanced tree methods such as RF and GBRT tend to overfit, so their performance is slightly worse. By contrast, for model (b), these methods clearly dominate Lasso and ENet, because the latter cannot capture the nonlinearity in the model. GLM is slightly better, but is dominated by NNs, RF, and GBRT. OLS is the worst in all settings, not surprisingly. PLS outperforms PCR in the linear model (a), but is dominated in the nonlinear case. When $P_{c}$ increases, the IS $R^{2}$ tends to increase whereas the out-of-sample $R^{2}$ decreases. Hence, the performance of all methods deteriorates as overfitting exacerbates. Using Huber loss improves 
Table A.2: Comparison of Predictive $R^{2}$ s for Alternative Prediction Horizons in Simulations Model (a) (b)

\begin{tabular}{|c|c|c|c|c|c|c|c|c|c|c|c|c|}
\hline \multirow{2}{*}{$\frac{\text { Horizon }}{R^{2}(\%)}$} & \multicolumn{2}{|c|}{ Quarter } & \multicolumn{2}{|c|}{ Halfyear } & \multicolumn{2}{|c|}{ Annual } & \multicolumn{2}{|c|}{ Quarter } & \multicolumn{2}{|c|}{ Halfyear } & \multicolumn{2}{|c|}{ Annual } \\
\hline & IS & OOS & IS & OOS & IS & OOS & IS & OOS & IS & OOS & IS & OOS \\
\hline OLS & 18.84 & -0.90 & 27.67 & 0.19 & 35.40 & -0.15 & 10.03 & -16.47 & 15.02 & -23.48 & 20.19 & -30.73 \\
\hline $\mathrm{OLS}+\mathrm{H}$ & 18.82 & -0.76 & 27.66 & 0.31 & 35.38 & -0.09 & 10.00 & -16.27 & 14.99 & -23.32 & 20.15 & -30.66 \\
\hline PCR & 3.86 & 0.91 & 5.50 & 1.32 & 7.58 & 1.39 & 0.90 & -0.04 & 1.30 & -0.04 & 1.71 & -0.24 \\
\hline PLS & 15.12 & 6.56 & 21.52 & 8.40 & 26.46 & 8.14 & 1.91 & -0.42 & 1.73 & -0.33 & 2.78 & -0.80 \\
\hline Lasso & 14.10 & 10.33 & 20.42 & 14.68 & 25.06 & 16.76 & 3.10 & 1.17 & 4.03 & 1.12 & 4.87 & 0.40 \\
\hline Lasso $+\mathrm{H}$ & 14.01 & 10.32 & 20.30 & 14.67 & 24.85 & 16.74 & 3.03 & 1.19 & 3.91 & 1.15 & 4.66 & 0.48 \\
\hline Ridge & 15.76 & 7.81 & 23.26 & 10.67 & 29.27 & 11.65 & 4.07 & 0.43 & 5.66 & 0.44 & 6.72 & 0.00 \\
\hline Ridge+H & 15.68 & 7.84 & 23.15 & 10.69 & 29.13 & 11.65 & 4.00 & 0.45 & 5.56 & 0.46 & 6.56 & 0.04 \\
\hline ENet & 14.08 & 10.33 & 20.49 & 14.69 & 25.06 & 16.69 & 3.10 & 1.15 & 4.07 & 1.14 & 4.80 & 0.41 \\
\hline ENet+H & 13.99 & 10.32 & 20.37 & 14.68 & 24.85 & 16.67 & 3.02 & 1.17 & 3.95 & 1.18 & 4.60 & 0.48 \\
\hline GLM & 13.90 & 9.40 & 21.02 & 13.53 & 27.15 & 15.36 & 7.61 & 2.46 & 10.79 & 2.88 & 13.07 & 1.63 \\
\hline $\mathrm{GLM}+\mathrm{H}$ & 13.79 & 9.42 & 20.88 & 13.56 & 26.99 & 15.40 & 7.48 & 2.51 & 10.59 & 2.93 & 12.77 & 1.71 \\
\hline $\mathrm{RF}$ & 17.56 & 8.11 & 25.24 & 11.86 & 31.04 & 14.32 & 15.52 & 5.91 & 20.53 & 7.11 & 22.48 & 6.05 \\
\hline GBRT & 15.98 & 8.94 & 22.68 & 13.27 & 28.68 & 15.06 & 12.39 & 5.87 & 15.85 & 6.90 & 18.08 & 5.99 \\
\hline $\mathrm{GBRT}+\mathrm{H}$ & 15.70 & 8.78 & 22.84 & 13.45 & 29.07 & 15.29 & 12.12 & 5.87 & 16.00 & 7.13 & 18.20 & 6.17 \\
\hline NN1 & 15.68 & 9.99 & 23.04 & 14.07 & 29.62 & 15.58 & 13.25 & 5.36 & 17.95 & 6.29 & 20.68 & 5.32 \\
\hline NN2 & 15.56 & 9.96 & 22.72 & 14.00 & 28.90 & 16.01 & 13.29 & 5.76 & 17.95 & 6.78 & 20.10 & 5.43 \\
\hline NN3 & 15.45 & 9.98 & 22.66 & 13.94 & 28.59 & 16.10 & 13.11 & 5.57 & 17.50 & 6.63 & 20.31 & 5.27 \\
\hline NN4 & 15.49 & 9.91 & 22.32 & 14.06 & 28.59 & 15.97 & 13.20 & 5.56 & 17.90 & 6.52 & 19.67 & 5.20 \\
\hline NN5 & 15.19 & 9.82 & 22.14 & 13.85 & 28.22 & 15.92 & 13.00 & 5.24 & 17.15 & 6.19 & 18.86 & 5.08 \\
\hline Oracle & 14.37 & 12.72 & 20.73 & 18.15 & 25.42 & 21.56 & 10.91 & 10.28 & 13.61 & 12.75 & 13.04 & 11.52 \\
\hline
\end{tabular}

Note: In this table, we report the average in-sample (IS) and out-of-sample (OOS) $R^{2}$ s for models $(a)$ and $(b)$ using Ridge, Lasso, Elastic Net (ENet), generalized linear model with group lasso (GLM), random forest (RF), gradient boosted regression trees (GBRT), and five architectures of neural networks (NN1,..,NN5), respectively. "+H" indicates the use of Huber loss instead of the $l_{2}$ loss. "Oracle" stands for using the true covariates in a pooled-OLS regression. We fix $N=200, T=180, P_{x}=2$ and $P_{c}=100$, comparing the performance of different horizons. The number of Monte Carlo repetitions is 100 .

the out-of-sample performance for almost all methods. RF, GBRT plus Huber loss remain the best choices for the nonlinear model. The comparison among NNs demonstrates a stark trade-off between model flexibility and implementation difficulty. Deeper models potentially allow for more parsimonious representation of the data, but their objective functions are more involved to optimize. For instance, the APG algorithm used in Elastic Net is not feasible for NNs, because its loss (as a function of weight parameters) is non-convex. As shown in the table, shallower NNs tend to outperform.

Table A.2 presents the same IS and OOS $R^{2}$ s for prediction conducted for different horizons, e.g., quarterly, half-yearly, and annually. We observe the usual increasing/hump-shape patterns of $R^{2}$ s against prediction horizons documented in the literature, which is driven by the persistence of covariates. The relative performance across different models maintains the same.

Next, we report the average variable selection frequencies of 6 particular covariates and the average of the remaining $P-6$ covariates for models $(a)$ and $(b)$ in Table A.3, using Lasso, Elastic Net, and Group Lasso and their robust versions. We focus on these methods because they all impose the $l_{1}$ penalty and hence encourage variable selection. As expected, for model $(a)$, the true covariates 
Table A.3: Comparison of Average Variable Selection Frequencies in Simulations

Model $(a)$

\begin{tabular}{|c|c|c|c|c|c|c|c|c|}
\hline Parameter & Method & $c_{i 1, t}$ & $c_{i 2, t}$ & $c_{i 3, t}$ & $c_{i 1, t} \times x_{t}$ & $c_{i 2, t} \times x_{t}$ & $c_{i 3, t} \times x_{t}$ & Noise \\
\hline \multirow[t]{6}{*}{$P_{c}=50$} & Lasso & 0.95 & 0.94 & 0.65 & 0.53 & 0.51 & 0.85 & 0.09 \\
\hline & Lasso+H & 0.95 & 0.94 & 0.63 & 0.53 & 0.50 & 0.86 & 0.08 \\
\hline & ENet & 0.95 & 0.94 & 0.65 & 0.54 & 0.51 & 0.86 & 0.09 \\
\hline & $\mathrm{ENet}+\mathrm{H}$ & 0.95 & 0.94 & 0.64 & 0.53 & 0.50 & 0.86 & 0.09 \\
\hline & GLM & 0.95 & 0.95 & 0.72 & 0.61 & 0.63 & 0.90 & 0.13 \\
\hline & $\mathrm{GLM}+\mathrm{H}$ & 0.95 & 0.94 & 0.70 & 0.61 & 0.62 & 0.90 & 0.12 \\
\hline \multirow[t]{6}{*}{$P_{c}=100$} & Lasso & 0.95 & 0.94 & 0.65 & 0.52 & 0.49 & 0.85 & 0.06 \\
\hline & Lasso $+\mathrm{H}$ & 0.95 & 0.94 & 0.63 & 0.53 & 0.49 & 0.86 & 0.06 \\
\hline & ENet & 0.95 & 0.94 & 0.65 & 0.53 & 0.49 & 0.86 & 0.06 \\
\hline & $\mathrm{ENet}+\mathrm{H}$ & 0.95 & 0.94 & 0.64 & 0.53 & 0.49 & 0.86 & 0.06 \\
\hline & GLM & 0.95 & 0.94 & 0.72 & 0.58 & 0.61 & 0.90 & 0.09 \\
\hline & $\mathrm{GLM}+\mathrm{H}$ & 0.95 & 0.94 & 0.69 & 0.55 & 0.60 & 0.90 & 0.09 \\
\hline \multicolumn{9}{|c|}{ Model (b) } \\
\hline Parameter & Method & $c_{i 1, t}$ & $c_{i 2, t}$ & $c_{i 3, t}$ & $c_{i 1, t} \times x_{t}$ & $c_{i 2, t} \times x_{t}$ & $c_{i 3, t} \times x_{t}$ & Noise \\
\hline \multirow[t]{6}{*}{$P_{c}=50$} & Lasso & 0.26 & 0.26 & 0.39 & 0.27 & 0.31 & 0.75 & 0.04 \\
\hline & Lasso+H & 0.25 & 0.25 & 0.38 & 0.28 & 0.31 & 0.75 & 0.04 \\
\hline & ENet & 0.26 & 0.25 & 0.39 & 0.27 & 0.31 & 0.76 & 0.04 \\
\hline & $\mathrm{ENet}+\mathrm{H}$ & 0.25 & 0.24 & 0.39 & 0.28 & 0.31 & 0.75 & 0.04 \\
\hline & GLM & 0.80 & 0.54 & 0.68 & 0.68 & 0.64 & 0.82 & 0.21 \\
\hline & $\mathrm{GLM}+\mathrm{H}$ & 0.79 & 0.54 & 0.70 & 0.68 & 0.62 & 0.82 & 0.20 \\
\hline \multirow[t]{6}{*}{$P_{c}=100$} & Lasso & 0.25 & 0.25 & 0.37 & 0.25 & 0.31 & 0.75 & 0.02 \\
\hline & Lasso+H & 0.24 & 0.24 & 0.36 & 0.26 & 0.31 & 0.75 & 0.02 \\
\hline & ENet & 0.25 & 0.25 & 0.37 & 0.25 & 0.31 & 0.76 & 0.02 \\
\hline & $\mathrm{ENet}+\mathrm{H}$ & 0.24 & 0.24 & 0.37 & 0.26 & 0.31 & 0.75 & 0.02 \\
\hline & GLM & 0.80 & 0.52 & 0.67 & 0.65 & 0.57 & 0.81 & 0.14 \\
\hline & $\mathrm{GLM}+\mathrm{H}$ & 0.79 & 0.48 & 0.67 & 0.66 & 0.56 & 0.81 & 0.13 \\
\hline
\end{tabular}

Note: In this table, we report the average variable selection frequencies of 6 particular covariates for models $(a)$ and $(b)$ (monthly horizon) using Lasso, Elastic Net (ENet), and generalized linear model with group lasso (GLM), respectively. " $+\mathrm{H}$ " indicates the use of Huber loss instead of the $l_{2}$ loss. Column "Noise" reports the average selection frequency of the remaining $P-6$ covariates. We fix $N=200, T=180$, and $P_{x}=2$, comparing $P_{c}=100$ with $P_{c}=50$. The number of Monte Carlo repetitions is 100 .

$\left(c_{i 1, t}, c_{i 2, t}, c_{i 3, t} \times x_{t}\right)$ are selected in over $85 \%$ of the sample paths, whereas correlated yet redundant covariates $\left(c_{i 3, t}, c_{i 1, t} \times x_{t}, c_{i 2, t} \times x_{t}\right)$ are also selected in around $60 \%$ of the samples. By contrast, the remaining covariates are rarely selected. Although model selection mistakes are unavoidable, perhaps due to the tension between variable selection and prediction or for finite sample issues, the true covariates are part of the selected models with high probabilities. For model $(b)$, while no covariates are part of the true model, the 6 covariates we present are more relevant, and hence selected substantially more frequently than the remaining $P-6$ ones.

Finally, we report the average VIPs of the 6 particular covariates and the average of the remaining $P-6$ covariates for models $(a)$ and $(b)$ in Table A.4, using random forest $(\mathrm{RF})$ and gradient boosted regression trees (GBRT), along with neural networks. We find similar results for both models $(a)$ and $(b)$ that the 6 covariates we present are substantially more important than the remaining $P-6$ 
Table A.4: Comparison of Average Variable Importance in Simulations

Model $(a)$

\begin{tabular}{|c|c|c|c|c|c|c|c|c|}
\hline Parameter & Method & $c_{i 1, t}$ & $c_{i 2, t}$ & $c_{i 3, t}$ & $c_{i 1, t} \times x_{t}$ & $c_{i 2, t} \times x_{t}$ & $c_{i 3, t} \times x_{t}$ & Noise \\
\hline \multirow[t]{8}{*}{$P_{c}=50$} & $\mathrm{RF}$ & 21.54 & 23.20 & 5.89 & 6.44 & 6.42 & 19.45 & 0.18 \\
\hline & GBRT & 23.86 & 27.86 & 5.64 & 6.41 & 6.03 & 25.66 & 0.05 \\
\hline & $\mathrm{GBRT}+\mathrm{H}$ & 24.01 & 27.43 & 5.33 & 6.30 & 6.78 & 25.88 & 0.05 \\
\hline & NN1 & 26.50 & 29.55 & 5.31 & 2.99 & 3.75 & 25.18 & 0.07 \\
\hline & NN2 & 26.35 & 28.93 & 5.04 & 3.12 & 3.93 & 25.74 & 0.07 \\
\hline & NN3 & 26.05 & 28.61 & 5.03 & 3.09 & 3.89 & 25.57 & 0.08 \\
\hline & NN4 & 26.09 & 28.72 & 5.08 & 3.37 & 3.82 & 25.59 & 0.08 \\
\hline & NN5 & 25.95 & 28.40 & 5.12 & 3.31 & 3.73 & 25.36 & 0.09 \\
\hline \multirow[t]{8}{*}{$P_{c}=100$} & $\mathrm{RF}$ & 21.40 & 23.08 & 5.84 & 5.62 & 5.87 & 19.18 & 0.10 \\
\hline & GBRT & 23.87 & 27.82 & 5.32 & 6.20 & 5.87 & 25.43 & 0.03 \\
\hline & $\mathrm{GBRT}+\mathrm{H}$ & 23.75 & 27.12 & 5.20 & 6.04 & 6.30 & 26.00 & 0.03 \\
\hline & NN1 & 25.78 & 28.36 & 5.03 & 2.77 & 3.60 & 24.57 & 0.05 \\
\hline & NN2 & 25.30 & 27.88 & 4.85 & 2.95 & 3.52 & 24.58 & 0.06 \\
\hline & NN3 & 25.32 & 28.03 & 4.73 & 2.89 & 3.50 & 24.53 & 0.06 \\
\hline & NN4 & 25.02 & 27.63 & 4.77 & 2.92 & 3.49 & 24.34 & 0.06 \\
\hline & NN5 & 24.78 & 27.73 & 4.82 & 3.07 & 3.54 & 24.19 & 0.06 \\
\hline \multicolumn{9}{|c|}{ Model $(b)$} \\
\hline Parameter & Method & $c_{i 1, t}$ & $c_{i 2, t}$ & $c_{i 3, t}$ & $c_{i 1, t} \times x_{t}$ & $c_{i 2, t} \times x_{t}$ & $c_{i 3, t} \times x_{t}$ & Noise \\
\hline \multirow[t]{8}{*}{$P_{c}=50$} & $\mathrm{RF}$ & 27.70 & 6.47 & 5.03 & 8.02 & 5.00 & 32.13 & 0.17 \\
\hline & GBRT & 31.24 & 7.37 & 5.82 & 8.81 & 6.43 & 36.41 & 0.04 \\
\hline & $\mathrm{GBRT}+\mathrm{H}$ & 32.05 & 7.46 & 5.83 & 8.87 & 6.62 & 35.57 & 0.04 \\
\hline & NN1 & 55.55 & 14.50 & 4.53 & 3.46 & 2.97 & 12.11 & 0.07 \\
\hline & NN2 & 51.84 & 13.66 & 4.15 & 2.96 & 2.72 & 18.32 & 0.07 \\
\hline & NN3 & 52.00 & 13.64 & 4.36 & 2.93 & 2.89 & 16.63 & 0.08 \\
\hline & NN4 & 51.07 & 13.61 & 4.45 & 3.31 & 2.81 & 16.19 & 0.09 \\
\hline & NN5 & 49.74 & 13.68 & 4.48 & 3.28 & 2.86 & 15.91 & 0.11 \\
\hline \multirow[t]{8}{*}{$P_{c}=100$} & $\mathrm{RF}$ & 26.42 & 5.74 & 4.40 & 7.77 & 4.69 & 31.93 & 0.10 \\
\hline & GBRT & 31.49 & 7.30 & 5.38 & 8.61 & 6.17 & 36.70 & 0.02 \\
\hline & $\mathrm{GBRT}+\mathrm{H}$ & 32.13 & 7.48 & 5.71 & 8.66 & 6.30 & 35.87 & 0.02 \\
\hline & NN1 & 53.09 & 13.53 & 4.79 & 3.45 & 2.77 & 12.11 & 0.05 \\
\hline & NN2 & 50.25 & 12.93 & 4.26 & 2.87 & 2.45 & 17.31 & 0.05 \\
\hline & NN3 & 50.36 & 13.00 & 4.30 & 2.90 & 2.54 & 15.43 & 0.06 \\
\hline & NN4 & 48.28 & 13.05 & 4.50 & 3.21 & 2.63 & 15.05 & 0.07 \\
\hline & NN5 & 43.44 & 12.41 & 4.71 & 3.61 & 2.59 & 16.09 & 0.09 \\
\hline
\end{tabular}

Note: In this table, we report the average variable importance of 6 particular covariates for models $(a)$ and $(b)$ (monthly horizon) using random forest (RF), gradient boosted regression trees (GBRT), and five architectures of neural networks $(\mathrm{NN} 1, \ldots, \mathrm{NN} 5)$, respectively. "+H" indicates the use of Huber loss instead of the $l_{2}$ loss. Column "Noise" reports the average variable importance of the remaining $P-6$ covariates. We fix $N=200, T=180$, and $P_{x}=2$, comparing $P_{c}=100$ with $P_{c}=50$. The number of Monte Carlo repetitions is 100 .

ones. All methods work equally well.

Overall, the simulation results suggest that the machine learning methods are successful in singling out informative variables, even though highly correlated covariates are difficult to distinguish. This is not surprising, as these methods are implemented to improve prediction, for which purpose the best model often does not agree with the true model, in particular when covariates are highly 
correlated.

\section{B Algorithms in Details}

\section{B.1 Lasso, Ridge, Elastic Net, and Group Lasso}

We present the accelerated proximal algorithm (APG), see, e.g., Parikh and Boyd (2013) and Polson et al. (2015)., which allows for efficient implementation of the elastic net, Lasso, Ridge regression, and Group Lasso for both $l_{2}$ and Huber losses. We rewrite their regularized objective functions as

$$
\mathcal{L}(\theta ; \cdot)=\underbrace{\mathcal{L}(\theta)}_{\text {Loss Function }}+\underbrace{\phi(\theta ; \cdot)}_{\text {Penalty }}
$$

where we omit the dependence on the tuning parameters. Specifically, we have

$$
\phi(\theta ; \cdot)= \begin{cases}\frac{1}{2} \lambda \sum_{j=1}^{P} \theta_{j}^{2}, & \text { Ridge; } \\ \lambda \sum_{j=1}^{P}\left|\theta_{j}\right|, & \text { Lasso; } \\ \lambda(1-\rho) \sum_{j=1}^{P}\left|\theta_{j}\right|+\frac{1}{2} \lambda \rho \sum_{j=1}^{P} \theta_{j}^{2}, & \text { Elastic Net; } \\ \lambda \sum_{j=1}^{P}\left\|\theta_{j}\right\|, & \text { Group Lasso. }\end{cases}
$$

where in the Group Lasso case, $\theta=\left(\theta_{1}, \theta_{2}, \ldots, \theta_{P}\right)$ is a $K \times P$ matrix.

Proximal algorithms are a class of algorithms for solving convex optimization problems, in which the base operation is evaluating the proximal operator of a function, ie., solving a small convex optimization problem. In many cases, this smaller problem has a closed form solution. The proximal operator is defined as:

$$
\operatorname{prox}_{\gamma f}(\theta)=\underset{z}{\operatorname{argmin}}\left\{f(z)+\frac{1}{2 \gamma}\|z-\theta\|^{2}\right\} .
$$

An important property of the proximal operator is that the minimizer of a convex function $f(\cdot)$ is a fixed point of $\operatorname{prox}_{f}(\cdot)$, i.e., $\theta^{\star}$ minimizes $f(\cdot)$ if and only if

$$
\theta^{\star}=\operatorname{prox}_{f}\left(\theta^{\star}\right) .
$$

The proximal gradient algorithm is designed to minimize an objective function of the form (B.3), where $\mathcal{L}(\theta)$ is differentiable function of $\theta$ but $\phi(\theta ; \cdot)$ is not. Using properties of the proximal operator, 
one can show that $\theta^{\star}$ minimizes (B.3), if and only if

$$
\theta^{\star}=\operatorname{prox}_{\gamma \phi}\left(\theta^{\star}-\gamma \nabla \mathcal{L}\left(\theta^{\star}\right)\right)
$$

This result motivates the first two iteration steps in Algorithm 1. The third step inside the while loop is a Nesterov momentum (Nesterov (1983)) adjustment that accelerates convergence.

The optimization problem requires the proximal operators of $\phi(\theta ; \cdot) \mathrm{s}$ in $(\mathrm{B} .4)$, which have closed forms:

$$
\operatorname{prox}_{\gamma \phi}(\theta)= \begin{cases}\frac{\theta}{1+\lambda \gamma}, & \text { Ridge; } \\ \lambda S(\theta, \lambda \gamma), & \text { Lasso; } \\ \frac{1}{1+\lambda \gamma \rho} S(\theta,(1-\rho) \lambda \gamma), & \text { Elastic Net; } \\ \left(\widetilde{S}\left(\theta_{1}, \lambda \gamma\right)^{\top}, \widetilde{S}\left(\theta_{2}, \lambda \gamma\right)^{\top}, \ldots, \widetilde{S}\left(\theta_{P}, \lambda \gamma\right)^{\top}\right)^{\top}, & \text { Group Lasso. }\end{cases}
$$

where $S(x, \mu)$ and $\widetilde{S}(x, \mu)$ are vector-valued functions, whose $i$ th components are defined by:

$$
(S(x, \mu))_{i}=\left\{\begin{array}{ll}
x_{i}-\mu, & \text { if } x_{i}>0 \text { and } \mu<\left|x_{i}\right| ; \\
x_{i}+\mu, & \text { if } x_{i}<0 \text { and } \mu<\left|x_{i}\right| ;, \\
0, & \text { if } \mu \geq\left|x_{i}\right| .
\end{array} \quad(\widetilde{S}(x, \mu))_{i}=\left\{\begin{array}{ll}
x_{i}-\mu \frac{x_{i}}{\left\|x_{i}\right\|}, & \text { if }\left\|x_{i}\right\|>\mu ; \\
0, & \text { if }\left\|x_{i}\right\| \leq \mu .
\end{array} .\right.\right.
$$

Note that $S(x, \mu)$ is the soft-thresholding operator, so the proximal algorithm is equivalent to the coordinate descent algorithm in the case of $l_{2}$ loss, see, e.g., Daubechies et al. (2004), Friedman et al. (2007). The proximal framework we adopt here allows efficient implementation of Huber loss and convergence acceleration.

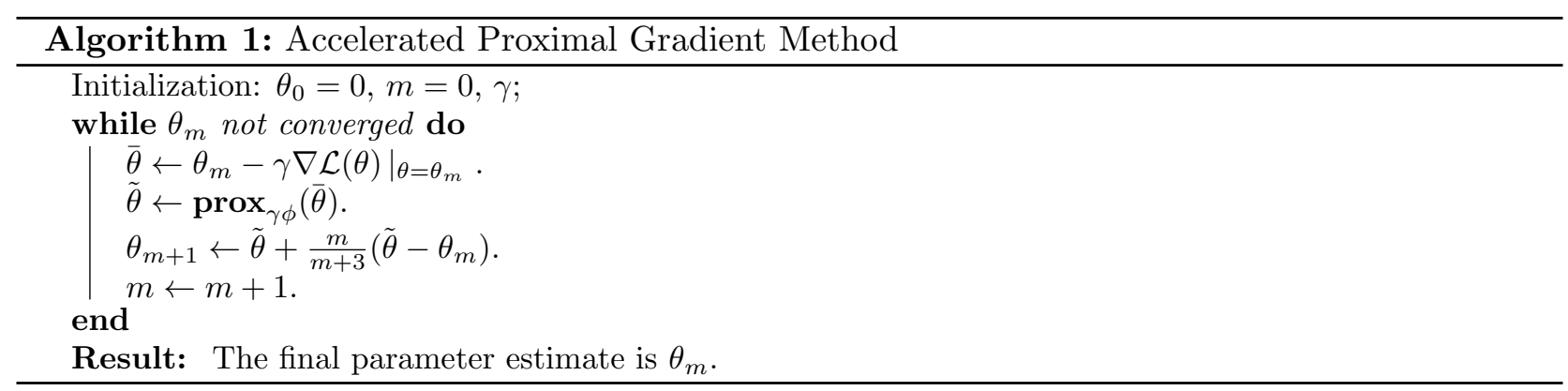




\section{B.2 Tree, Random Forest, and Gradient Boosted Tree}

Algorithm 2 is a greedy algorithm, see, e.g., Breiman et al. (1984), to grow a complete binary regression tree. Next, Algorithm 3 yields the random forest, e.g., Hastie et al. (2009). Finally, Algorithm 4 delivers the gradient boosted tree (Friedman (2001)), for which we follow the version written by Bühlmann and Hothorn (2007).

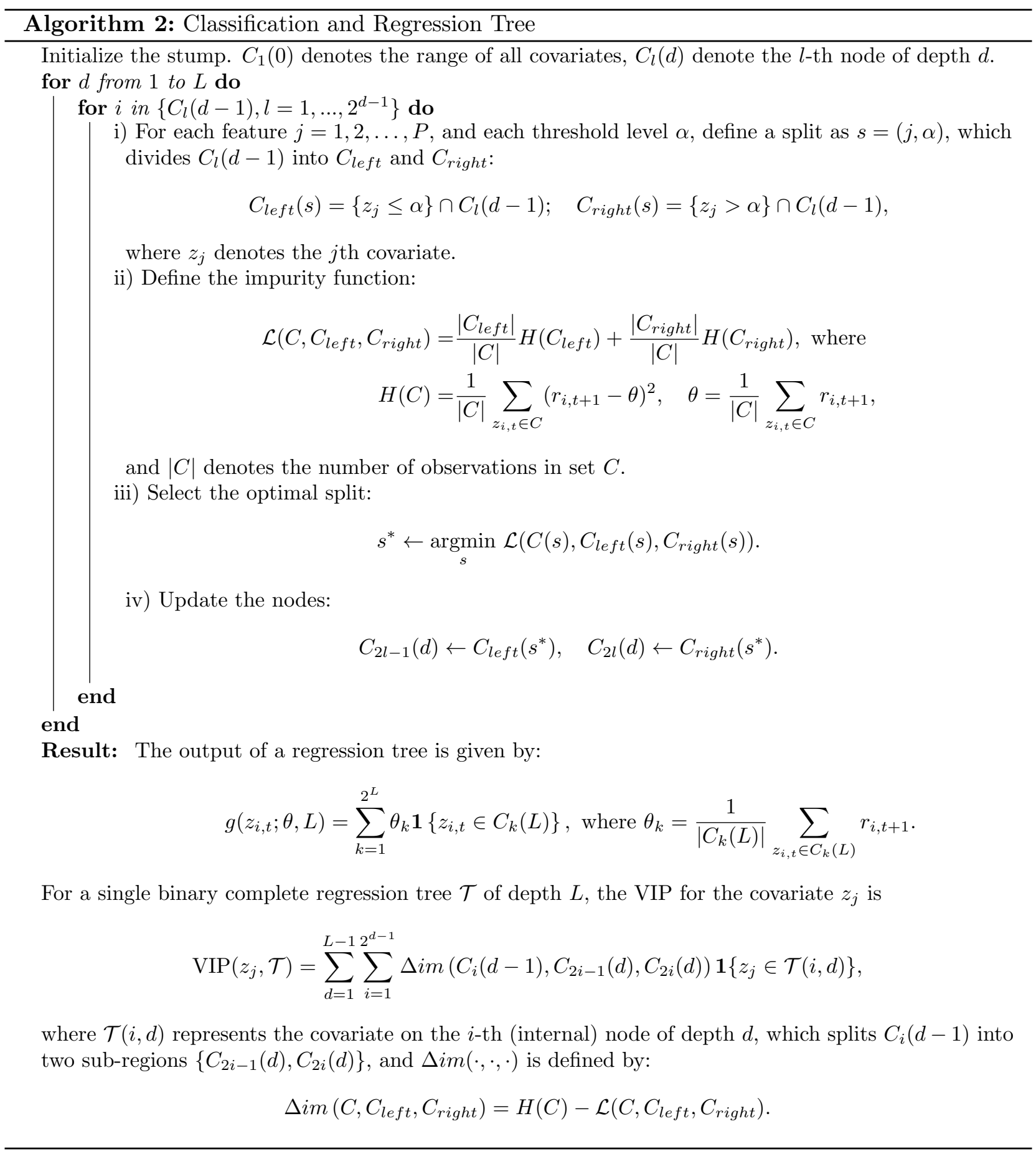



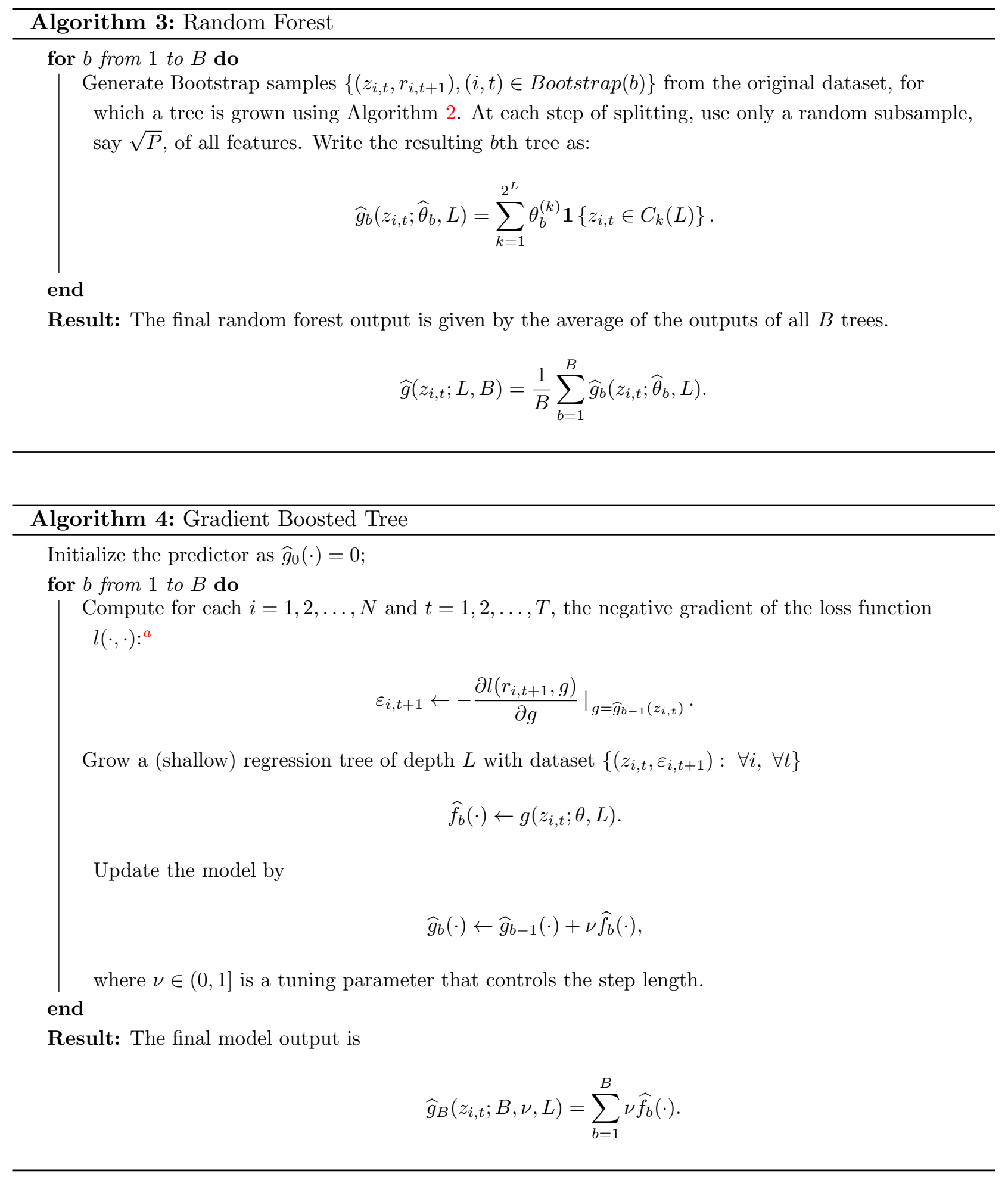

${ }^{a}$ The typical choice of $l(\cdot, \cdot)$ for regression is $l_{2}$ or Huber loss, whereas for classification, it is more common to use the following loss function:

$$
l(d, g(\cdot))=\log _{2}(1+\exp (-2(2 d-1) g(\cdot)))
$$




\section{B.3 Neural Networks}

It is common to fit the neural network using stochastic gradient descent (SGD), see, e.g., Goodfellow et al. (2016). We adopt the adaptive moment estimation algorithm (Adam), an efficient version of the SGD introduced by Kingma and Ba (2014). Adam computes adaptive learning rates for individual parameters using estimates of first and second moments of the gradients. We denote the loss function as $\mathcal{L}(\theta ; \cdot)$ and write $\mathcal{L}(\theta ; \cdot)=\frac{1}{T} \sum_{t=1}^{T} \mathcal{L}_{t}(\theta ; \cdot)$, where $\mathcal{L}_{t}(\theta ; \cdot)$ is the penalized cross-sectional average prediction error at time $t$. At each step of training, a batch sent to the algorithm is randomly sampled from the training dataset. Algorithm 6 is the early stopping algorithm that can be used in combination with many optimization routines, including Adam. Algorithm 7 gives the BatchNormalization transform (Ioffe and Szegedy (2015)), which we apply to each activation after ReLU transformation. Any neuron that previously receives a batch of $x$ as the input now receives $\mathrm{BN}_{\gamma, \beta}(x)$ instead, where $\gamma$ and $\beta$ are additional parameters to be optimized.

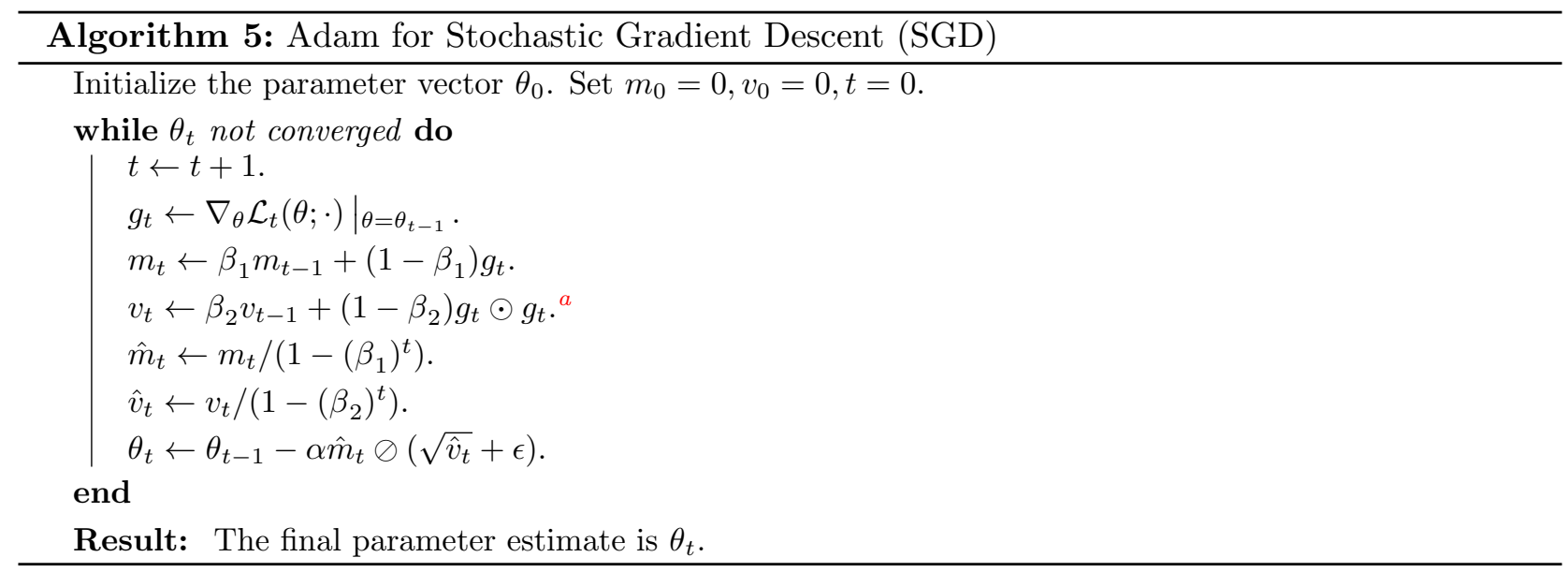

${ }^{a} \odot$ and $\oslash$ denote element-wise multiplication and division, respectively.

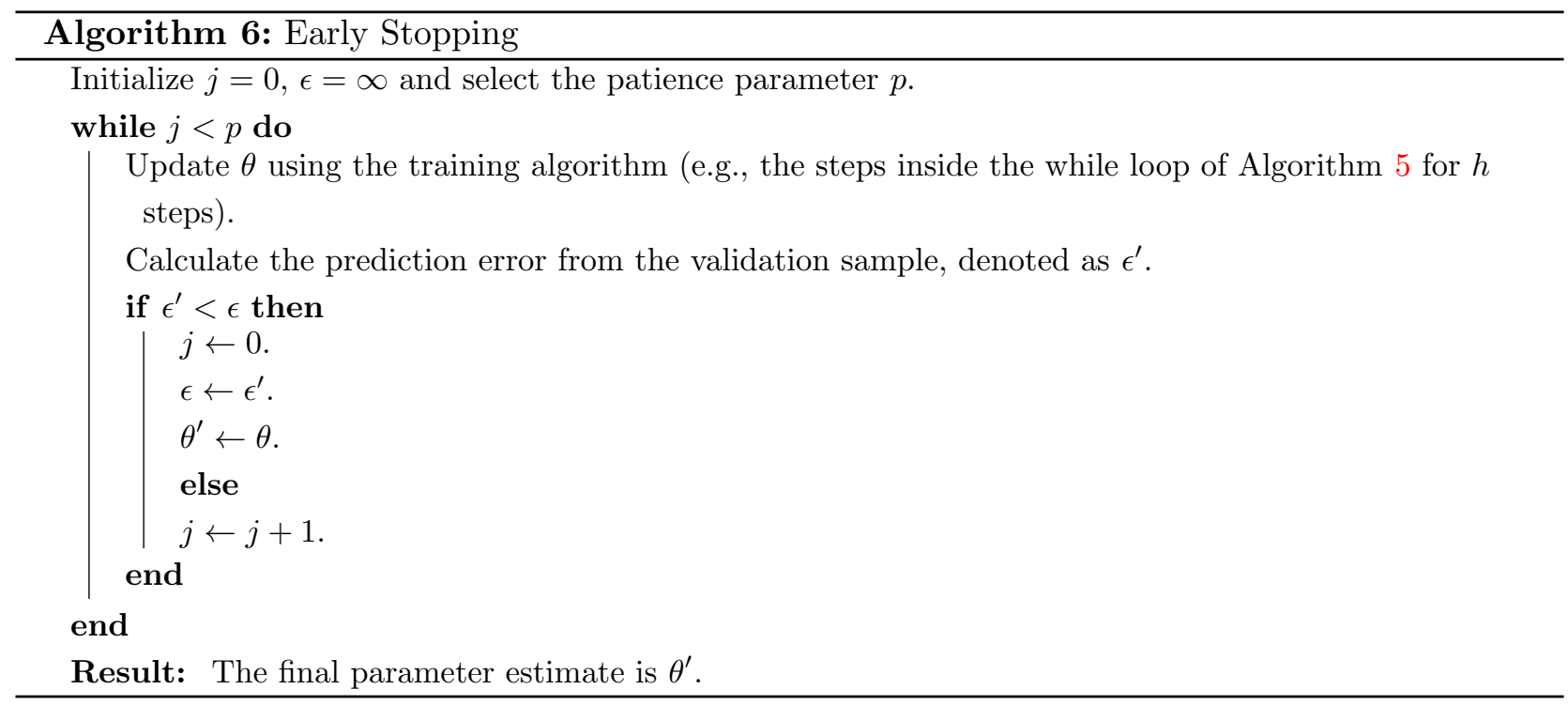




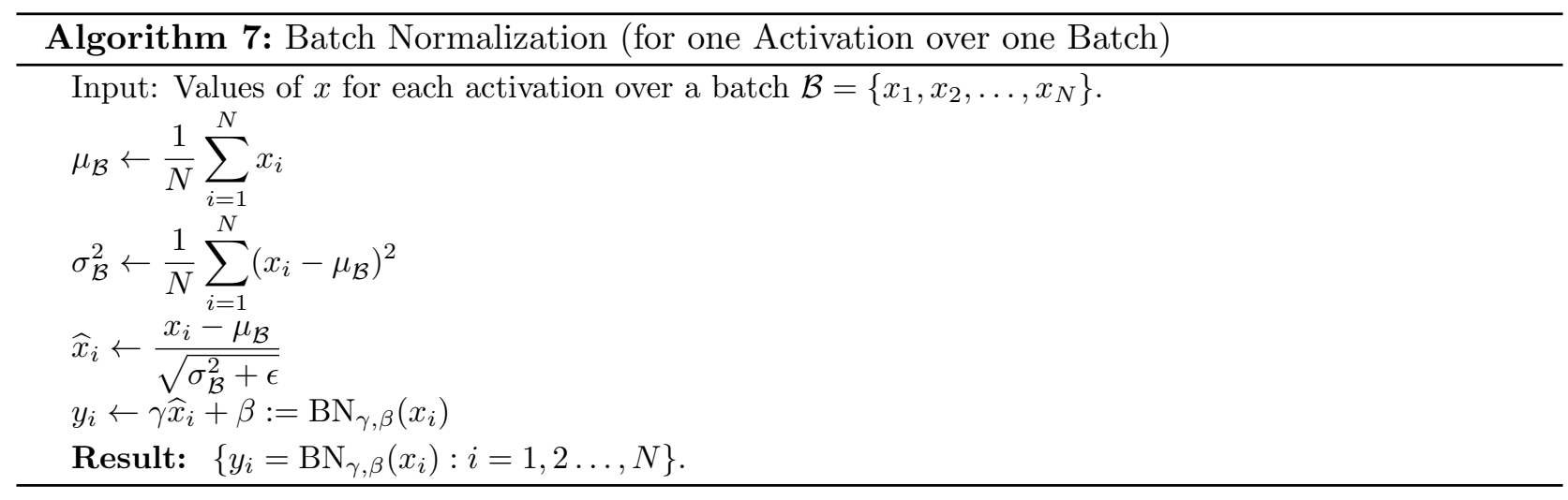

\section{Theoretical Properties of Machine Learning Models}

In this section, we provide references on the asymptotic properties of machine learning methods discussed in the main text. The references below are unavoidably selective and by no means complete. We invite interested readers to consult references within the following papers.

For theoretical properties of lasso, see Knight and Fu (2000), Bickel et al. (2009), Meinshausen and Yu (2009), Tibshirani (2011), Wainwright (2009), and Zhang and Huang (2008). And for elastic net, see Zou and Hastie (2005), Zou and Zhang (2009), and Mol et al. (2009). For group Lasso in linear models, see Lounici et al. (2011), and see Bach (2008) and Ravikumar et al. (2009) for additive and nonparametric models. While most theoretical analysis in high-dimensional statistics assume that data have sub-Gaussian or sub-exponential tails, Fan et al. (2017) provide a theoretical justification of using Huber's loss function in the high-dimensional setting.

For dimension reduction techniques, there is a large literature in statistics on the asymptotic behavior of PCA, e.g., Bai (1999), Johnstone (2001), Johnstone and Lu (2009), Paul (2007), Wang and Fan (2017), and another large literature in econometrics focusing on the asymptotic theory of PCA in modern factor analysis, e.g., Stock and Watson (2002), Bai and Ng (2002), and Bai and Ng (2013). There are, however, fewer results on the asymptotic analysis of PCR and PLS in particular. One can refer to Giglio and Xiu (2016) for the asymptotic theory of PCR in the context of risk premia estimation, and Kelly and Pruitt $(2013,2015)$ for the theory of PLS with its application to forecasting risk premia in financial markets.

A recent literature analyzes theoretical properties of random forests, see Biau (2012), Scornet et al. (2015), Mentch and Hooker (2016), Wager et al. (2014), and Wager and Athey (2018). The properties of gradient boosting, on the other hand, are well understood from the early work of e.g., Friedman et al. (2000b), Bühlmann and Yu (2003), Lugosi and Vayatis (2004), and Zhang and Yu (2005) for both classification and regression problems. However, much work remains to be done to fully take into account optimization and regularization algorithms that are essential to the desirable performance of various boosting methods, e.g., the popular XGBoost system designed by Chen and Guestrin (2016).

Likewise, theoretical properties of neural networks and deep learning are in large part underdeveloped (for an overview, see Fan et al., 2019). First, the approximation theory of neural networks 
is far from complete. Although earlier work have established a universal approximation theory with a single hidden layer network (e.g., Hornik et al., 1989), a recent line of work sheds light on the distinction between depth and width of a multi-layer network. Eldan and Shamir (2016) formally demonstrate that depth - even if increased by one layer - can be exponentially more valuable than increasing width in standard feed-forward neural networks (see also Lin et al., 2017; Rolnick and Tegmark, 2018).

Second, any theoretical understanding of neural networks should explicitly account for the modern optimization algorithms that, in combination with statistical analysis, are critical to their success. But training a deep neural network typically involves a grab bag of algorithms, e.g., SGD, Adam, batch normalization, skipping connections (He et al., 2016b), some of which rely on heuristic explanation without rigorous analysis. A promising recent strand of work Chizat and Bach (2018), Mei et al. (2018), and Mei et al. (2019) approximate the evolution of network weight parameters in the SDG algorithm for networks with a single hidden layer. They show that mean-field partial differential equations accurately describe this process as long as the number of hidden units is sufficiently large. In summary, there remains much work to be done to establish theoretical properties of deep learning.

\section{Sample Splitting}

We consider a number of sample splitting schemes studied in the forecast evaluation literature (see, e.g., West, 2006). The "fixed" scheme splits the data into training, validation, and testing samples. It estimates the model once from the training and validation samples, and attempts to fit all points in the testing sample using this fixed model estimate.

A common alternative to the fixed split scheme is a "rolling" scheme, in which the training and validation samples gradually shift forward in time to include more recent data, but holds the total number of time periods in each training and validation sample fixed. For each rolling window, one refits the model from the prevailing training and validation samples, and tracks a model's performance in the remaining test data that has not been subsumed by the rolling windows. The result is a sequence of performance evaluation measures corresponding to each rolling estimation window. This has the benefit of leveraging more recent information for prediction relative to the fixed scheme.

The third is a "recursive" performance evaluation scheme. Like the rolling approach, it gradually includes more recent observations in the training and validation windows. But the recursive scheme always retains the entire history in the training sample, thus its window size gradually increases. The rolling and recursive schemes are computationally expensive, in particular for more complicated models such as neural networks.

In our empirical exercise, we adopt a hybrid of these schemes by recursively increasing the training sample, periodically refitting the entire model once per year, and making out-of-sample predictions using the same fitted model over the subsequent year. Each time we refit, we increase the training sample by a year, while maintaining a fixed size rolling sample for validation. We choose to not cross-validate in order to maintain the temporal ordering of the data for prediction. 
Table A.5: Hyperparameters For All Methods

\begin{tabular}{|c|c|c|c|c|c|c|c|c|}
\hline & $\begin{array}{c}\text { OLS-3 } \\
+\mathrm{H}\end{array}$ & PLS & PCR & $\begin{array}{c}\text { ENet } \\
+\mathrm{H}\end{array}$ & $\begin{array}{c}\text { GLM } \\
+\mathrm{H}\end{array}$ & $\mathrm{RF}$ & $\begin{array}{c}\text { GBRT } \\
+\mathrm{H}\end{array}$ & NN1 - NN5 \\
\hline $\begin{array}{l}\text { Huber loss } \xi= \\
99.9 \% \text { quantile }\end{array}$ & $\checkmark$ & - & - & $\checkmark$ & $\checkmark$ & - & $\checkmark$ & - \\
\hline Others & & $K$ & $K$ & $\begin{array}{c}\rho=0.5 \\
\lambda \in\left(10^{-4}, 10^{-1}\right)\end{array}$ & $\begin{array}{c}\# \text { Knots }=3 \\
\lambda \in\left(10^{-4}, 10^{-1}\right)\end{array}$ & $\begin{array}{c}\text { Depth }=1 \sim 6 \\
\text { \#Trees }=300 \\
\text { \#Features in each split } \\
\in\{3,5,10,20,30,50 \ldots\}\end{array}$ & $\begin{array}{c}\text { Depth }=1 \sim 2 \\
\text { \#Trees }=1 \sim 1000 \\
\text { Learning Rate } \\
\operatorname{LR} \in\{0.01,0.1\}\end{array}$ & $\begin{array}{c}\text { L1 penalty } \\
\lambda_{1} \in\left(10^{-5}, 10^{-3}\right) \\
\text { Learning Rate } \\
\text { LR } \in\{0.001,0.01\} \\
\text { Batch Size }=10000 \\
\text { Epochs }=100 \\
\text { Patience }=5 \\
\text { Adam Para. }=\text { Default } \\
\text { Ensemble }=10\end{array}$ \\
\hline
\end{tabular}

Note: The table describes the hyperparameters that we tune in each machine learning method.

\section{E Hyperparameter Tuning}

Table A.5 describes the set of hyperparameters and their potential values used for tuning each machine learning model.

\section{F Additional Tables and Figures}




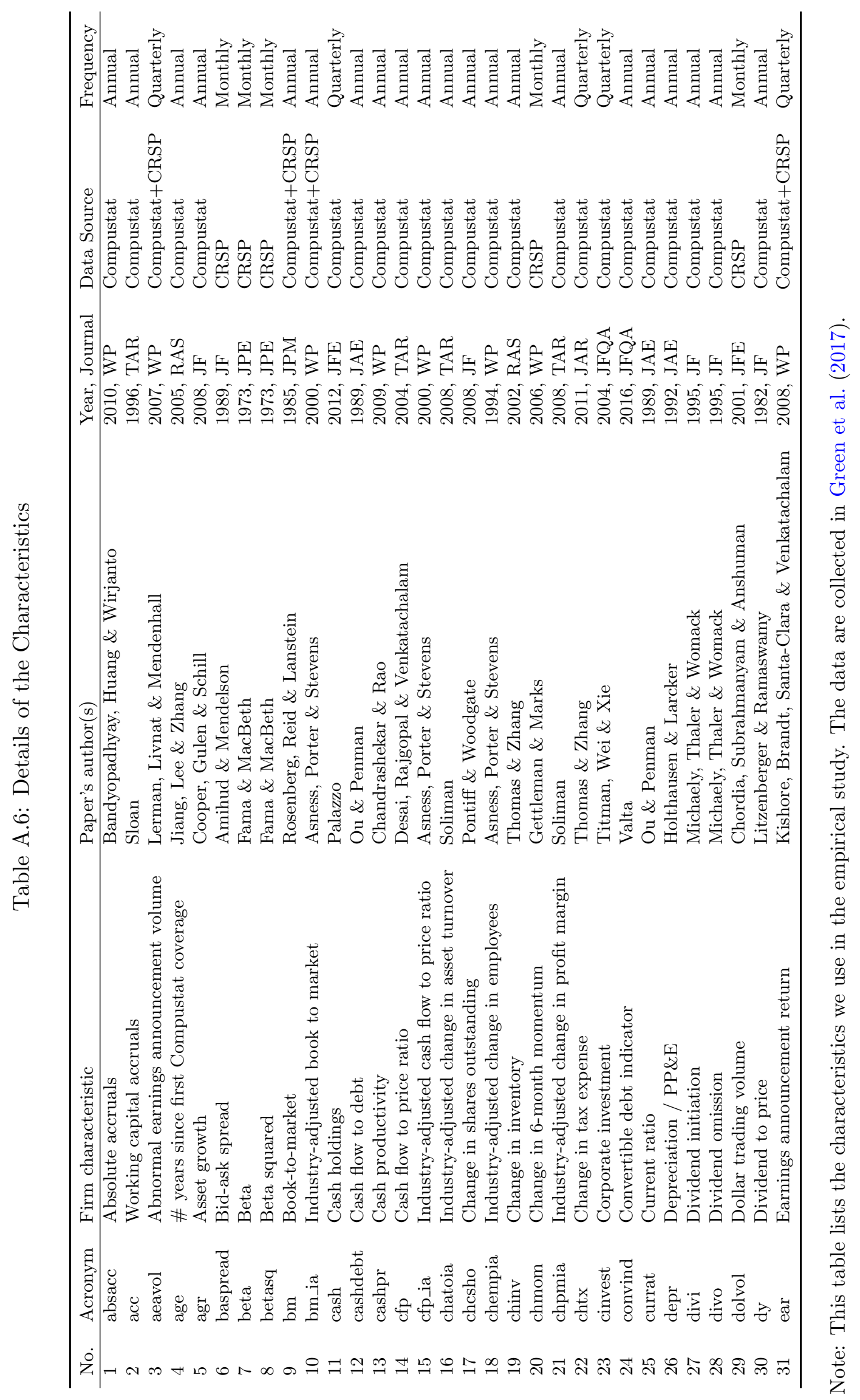




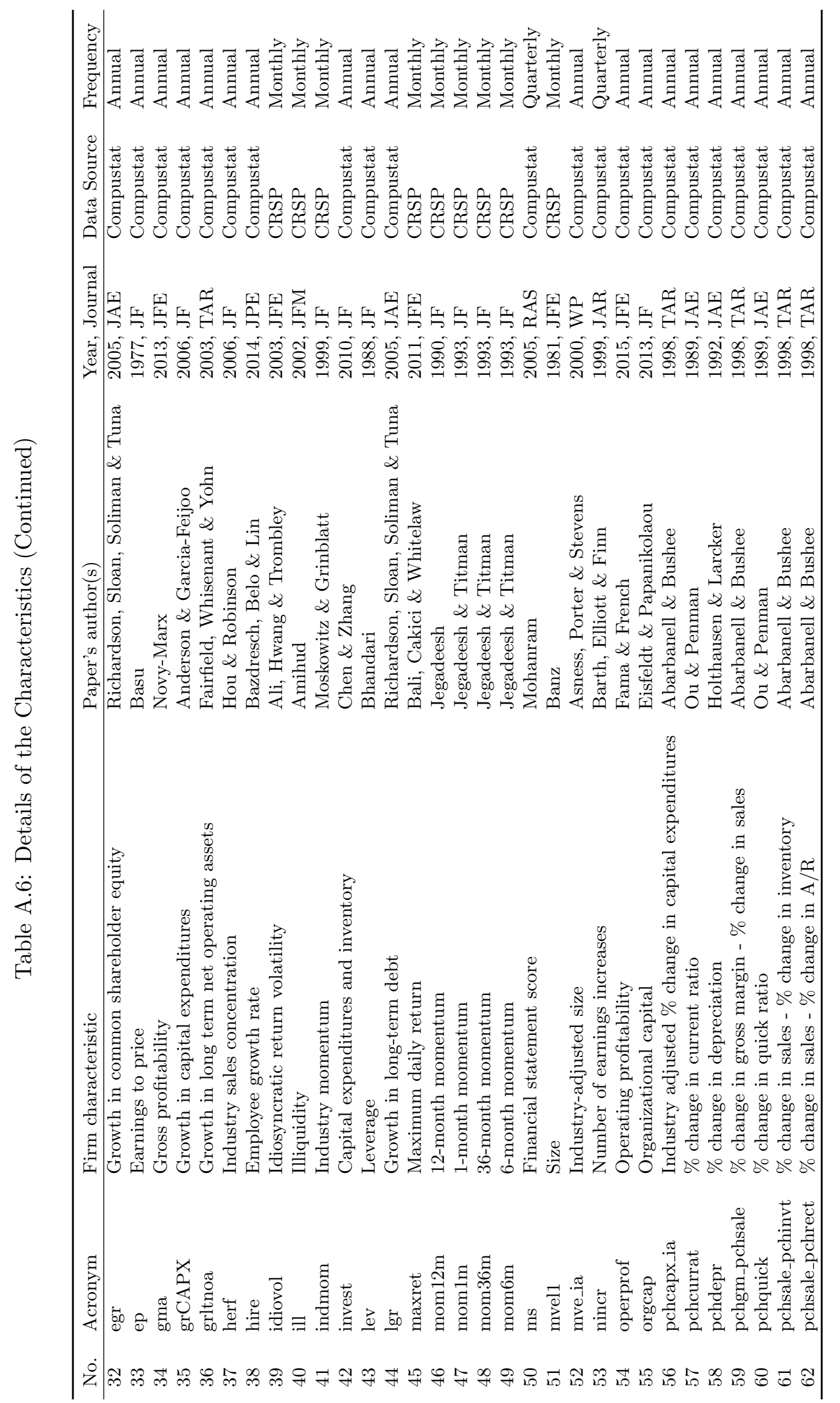




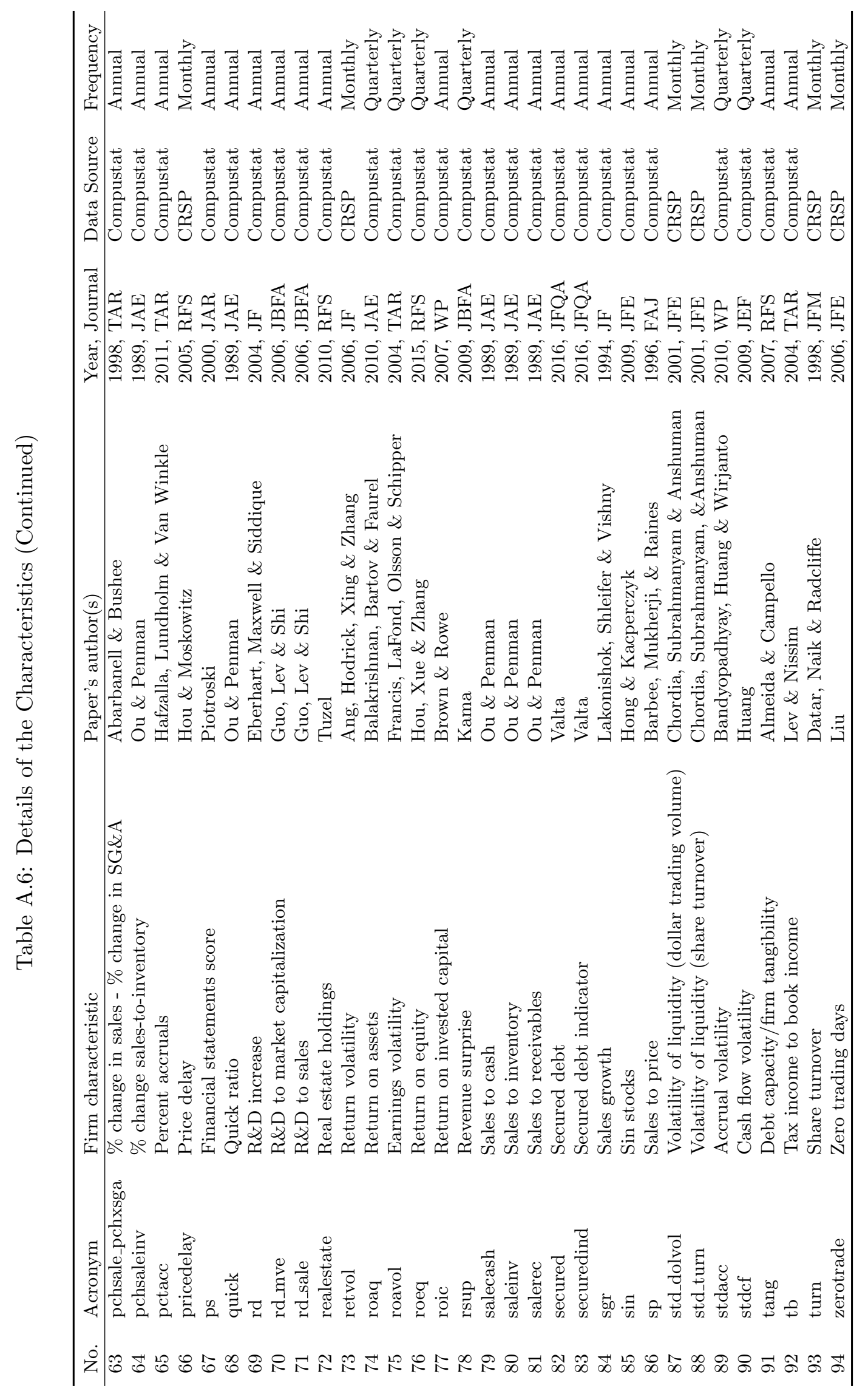


Table A.7: Implied Sharpe Ratio Improvements

\begin{tabular}{|c|c|c|c|c|c|c|c|c|c|c|c|c|}
\hline & $\begin{array}{c}\text { OLS-3 } \\
+\mathrm{H}\end{array}$ & PLS & PCR & $\begin{array}{c}\text { ENet } \\
+\mathrm{H}\end{array}$ & $\begin{array}{c}\text { GLM } \\
+\mathrm{H}\end{array}$ & $\mathrm{RF}$ & $\begin{array}{c}\text { GBRT } \\
+\mathrm{H}\end{array}$ & NN1 & NN2 & NN3 & NN4 & NN5 \\
\hline S\&P 500 & - & - & - & 0.08 & 0.08 & 0.14 & 0.15 & 0.11 & 0.12 & 0.20 & 0.17 & 0.12 \\
\hline Big Value & 0.05 & 0.00 & - & 0.03 & 0.07 & 0.14 & 0.12 & 0.09 & 0.10 & 0.15 & 0.13 & 0.11 \\
\hline Big Growth & - & - & - & 0.08 & 0.06 & 0.14 & 0.13 & 0.11 & 0.12 & 0.16 & 0.13 & 0.12 \\
\hline Big Neutral & 0.01 & - & - & 0.08 & 0.04 & 0.13 & 0.13 & 0.15 & 0.13 & 0.17 & 0.18 & 0.14 \\
\hline Small Value & 0.02 & 0.15 & 0.10 & 0.06 & 0.08 & 0.11 & 0.05 & 0.14 & 0.13 & 0.14 & 0.13 & 0.12 \\
\hline Small Growth & - & 0.03 & - & - & - & 0.14 & 0.21 & 0.01 & 0.09 & 0.10 & 0.08 & 0.10 \\
\hline Small Neutral & - & 0.06 & 0.02 & 0.02 & 0.03 & 0.09 & 0.04 & 0.06 & 0.06 & 0.07 & 0.06 & 0.07 \\
\hline Big Conservative & - & - & - & 0.09 & 0.04 & 0.10 & 0.05 & 0.11 & 0.10 & 0.14 & 0.12 & 0.10 \\
\hline Big Aggressive & - & - & - & 0.04 & 0.09 & 0.20 & 0.23 & 0.16 & 0.18 & 0.21 & 0.18 & 0.17 \\
\hline Big Neutral & - & - & - & 0.08 & 0.05 & 0.11 & 0.08 & 0.08 & 0.08 & 0.14 & 0.14 & 0.11 \\
\hline Small Conservative & - & 0.12 & 0.08 & 0.00 & 0.04 & 0.10 & 0.06 & 0.09 & 0.09 & 0.10 & 0.09 & 0.09 \\
\hline Small Aggressive & - & 0.09 & 0.00 & - & 0.03 & 0.16 & 0.22 & 0.06 & 0.11 & 0.12 & 0.10 & 0.12 \\
\hline Small Neutral & - & 0.04 & 0.01 & 0.04 & 0.03 & 0.07 & 0.00 & 0.06 & 0.06 & 0.08 & 0.06 & 0.07 \\
\hline Big Robust & - & - & - & 0.06 & 0.04 & 0.11 & 0.03 & 0.08 & 0.08 & 0.13 & 0.10 & 0.08 \\
\hline Big Weak & 0.03 & 0.15 & 0.12 & 0.10 & 0.12 & 0.14 & 0.19 & 0.19 & 0.19 & 0.21 & 0.17 & 0.17 \\
\hline Big Neutral & - & - & - & 0.06 & 0.02 & 0.14 & 0.12 & 0.11 & 0.13 & 0.15 & 0.15 & 0.13 \\
\hline Small Robust & - & 0.04 & - & 0.00 & - & 0.07 & 0.02 & 0.02 & 0.05 & 0.06 & 0.05 & 0.05 \\
\hline Small Weak & 0.04 & 0.17 & 0.11 & - & 0.08 & 0.17 & 0.22 & 0.13 & 0.15 & 0.16 & 0.15 & 0.15 \\
\hline Small Neutral & - & 0.01 & - & - & - & 0.06 & - & 0.01 & 0.03 & 0.04 & 0.03 & 0.04 \\
\hline Big Up & - & - & - & 0.06 & 0.11 & 0.11 & 0.08 & 0.07 & 0.07 & 0.10 & 0.10 & 0.09 \\
\hline Big Down & - & - & - & 0.05 & - & 0.13 & 0.08 & 0.04 & 0.08 & 0.12 & 0.10 & 0.10 \\
\hline Big Medium & - & - & - & 0.13 & - & 0.22 & 0.25 & 0.19 & 0.20 & 0.24 & 0.22 & 0.18 \\
\hline Small Up & - & 0.08 & 0.06 & - & 0.03 & 0.07 & 0.00 & 0.01 & 0.01 & 0.02 & 0.02 & 0.03 \\
\hline Small Down & - & 0.03 & - & 0.03 & 0.00 & 0.23 & 0.22 & 0.13 & 0.14 & 0.17 & 0.15 & 0.16 \\
\hline Small Medium & 0.01 & 0.08 & 0.02 & 0.06 & 0.04 & 0.12 & 0.11 & 0.11 & 0.11 & 0.12 & 0.10 & 0.10 \\
\hline
\end{tabular}

Note: Improvement in annualized Sharpe ratio $\left(S R^{*}-S R\right)$ implied by the full sample Sharpe ratio of each portfolio together with machine learning predictive $R_{\text {oos }}^{2}$ from Table 5. Cases with negative $R_{\text {oos }}^{2}$ imply a Sharpe ratio deterioration and are omitted. 
Figure A.1: Characteristic Importance over Time by NN3

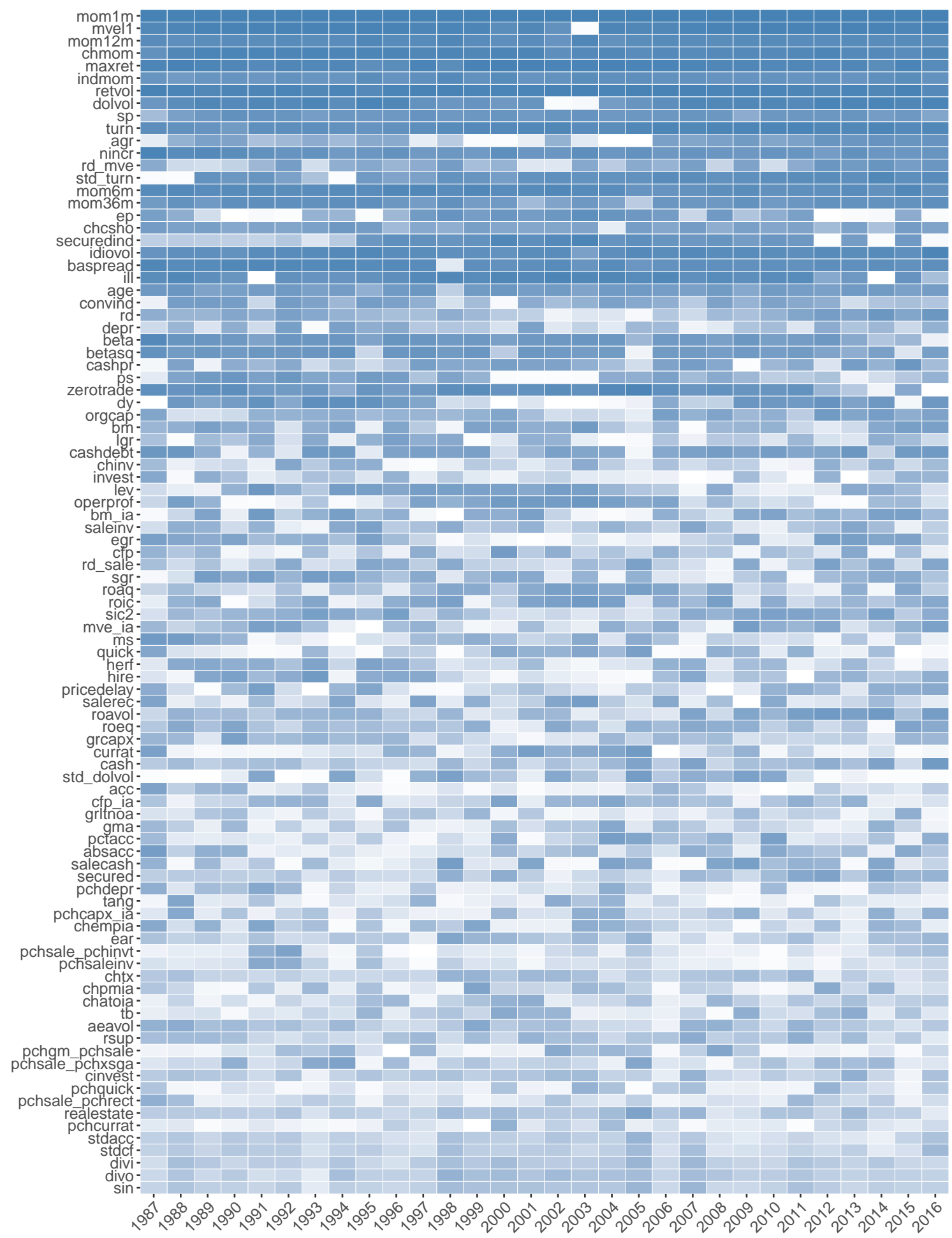

Note: This figure describes how NN3 ranks the 94 stock-level characteristics and the industry dummy (sic2) in terms of overall model contribution over 30 recursing training. Columns correspond to the year end of each of the 30 samples, and color gradients within each column indicate the most influential (dark blue) to least influential (white) variables. Characteristics are sorted in the same order of Figure 5. 
Figure A.2: Variable Importance Using SSD of Dimopoulos et al. (1995)

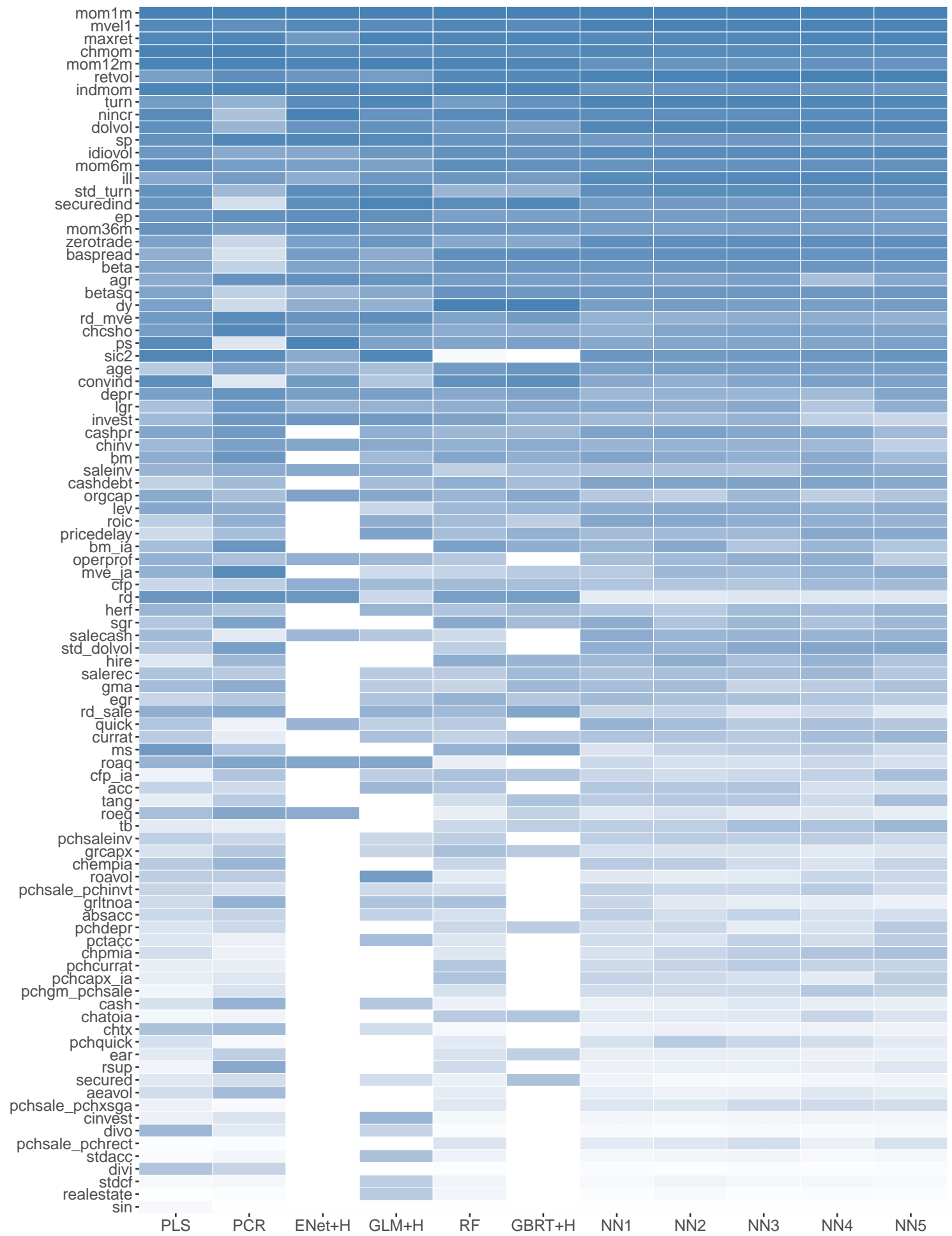

Note:

Rankings of 94 stock-level characteristics and the industry dummy (sic2) in terms of SSD. Characteristics are ordered based on the sum of their ranks over all models, with the most influential characteristics on top and least influential on bottom. Columns correspond to individual models, and color gradients within each column indicate the most influential (dark blue) to least influential (white) variables. 
Figure A.3: Characteristic Importance with Placebo Variables

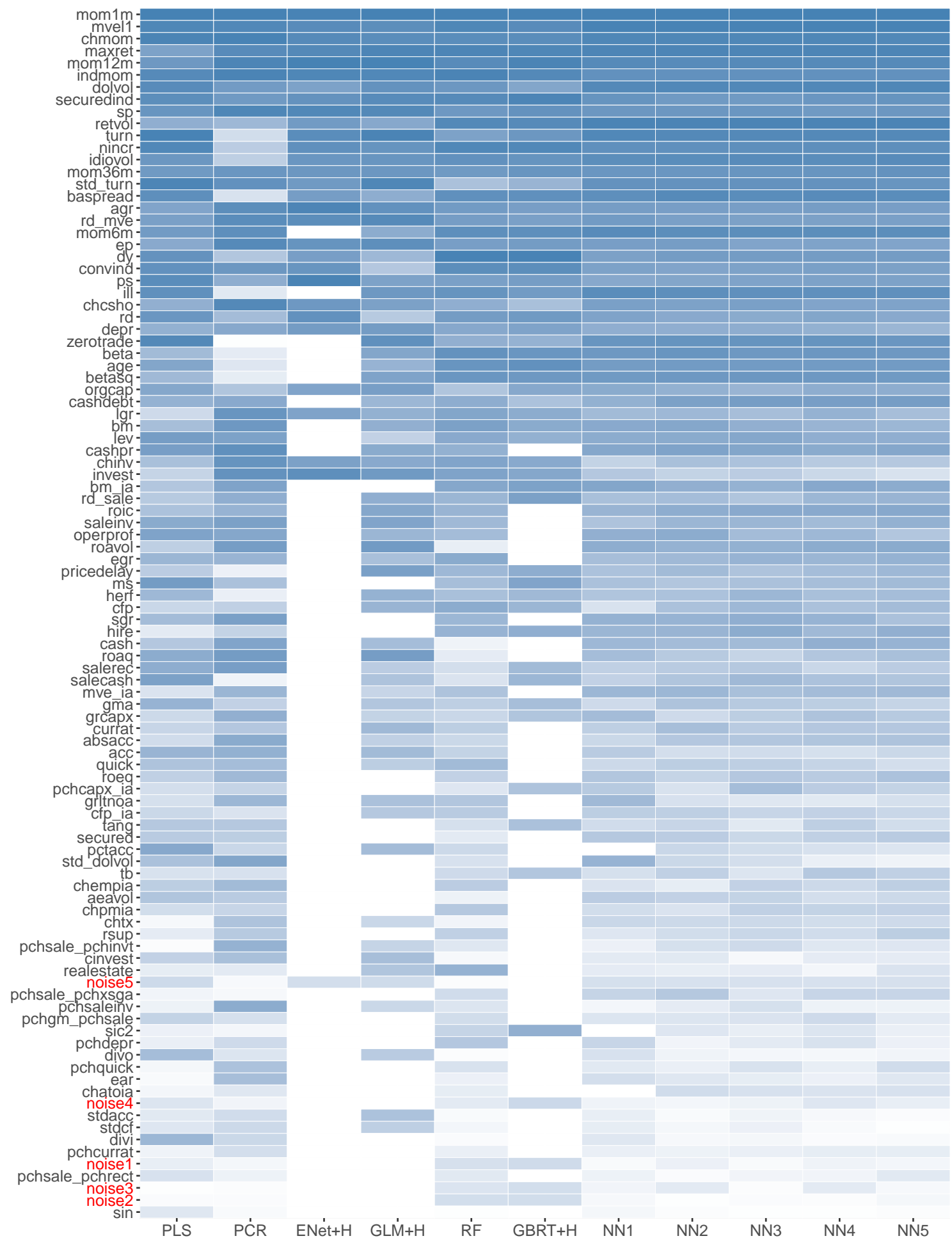

Note: This figure describes how each model ranks the 94 stock-level characteristics, the industry dummy (sic2), and five placebos in terms of overall model contribution. Columns correspond to individual models, and color gradients within each column indicate the most influential (dark blue) to least influential (white) variables. Characteristics are ordered based on the sum of their ranks over all models, with the most influential characteristics on top and least influential on bottom. 
Figure A.4: Stock/Macroeconomic Interactions

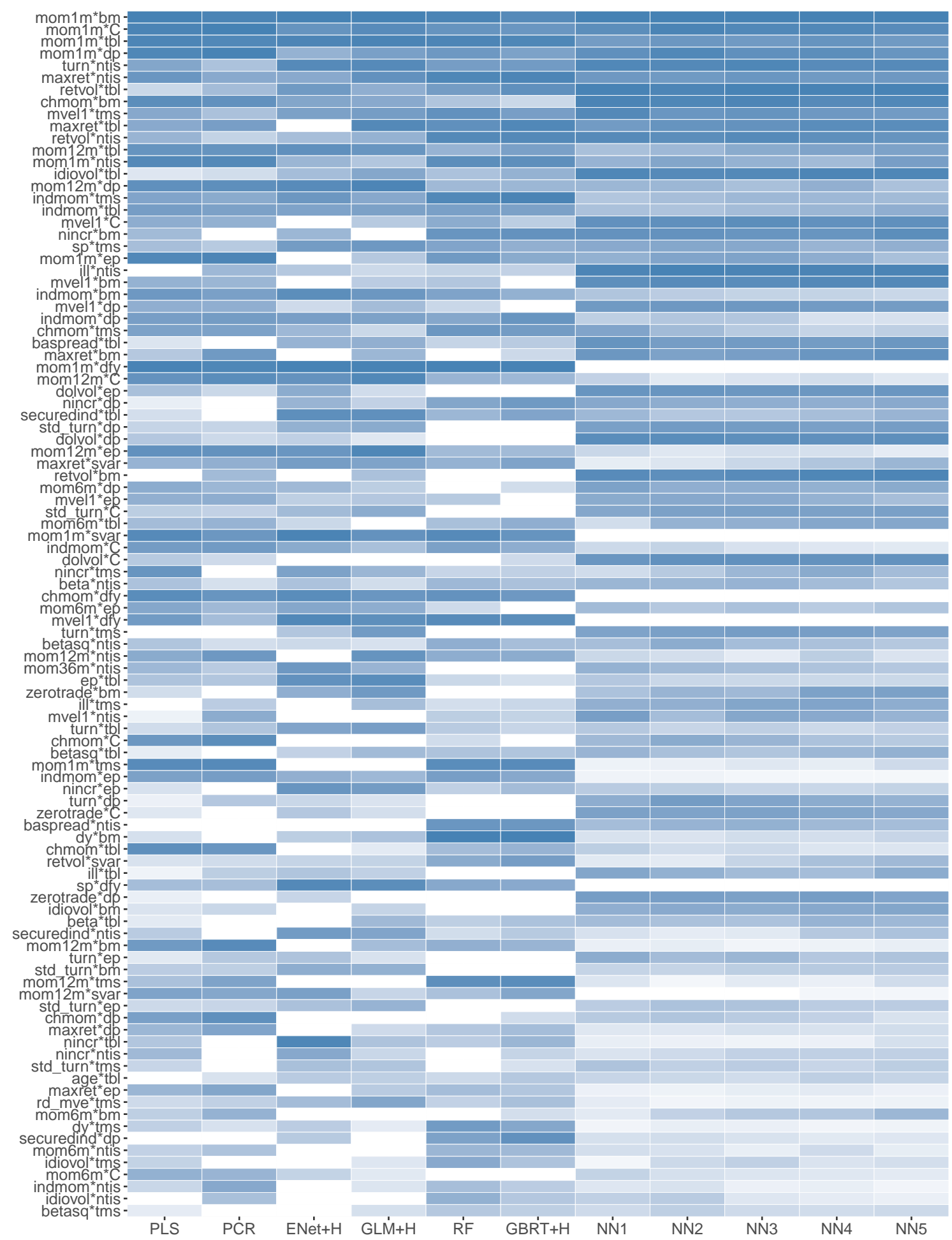

Note: Rankings of top 100 interactions between 94 stock-level stock characteristics and nine macro variables (including a constant, denoted $C$ ). Interactions are ordered based on the sum of their ranks over all models, with the most influential characteristics on top and least influential on bottom. Columns correspond to individual models, and color gradients within each column indicate the most influential (dark blue) to least influential (white) interactions. 
Figure A.5: Time Variation in Stock/Macroeconomic Interactions

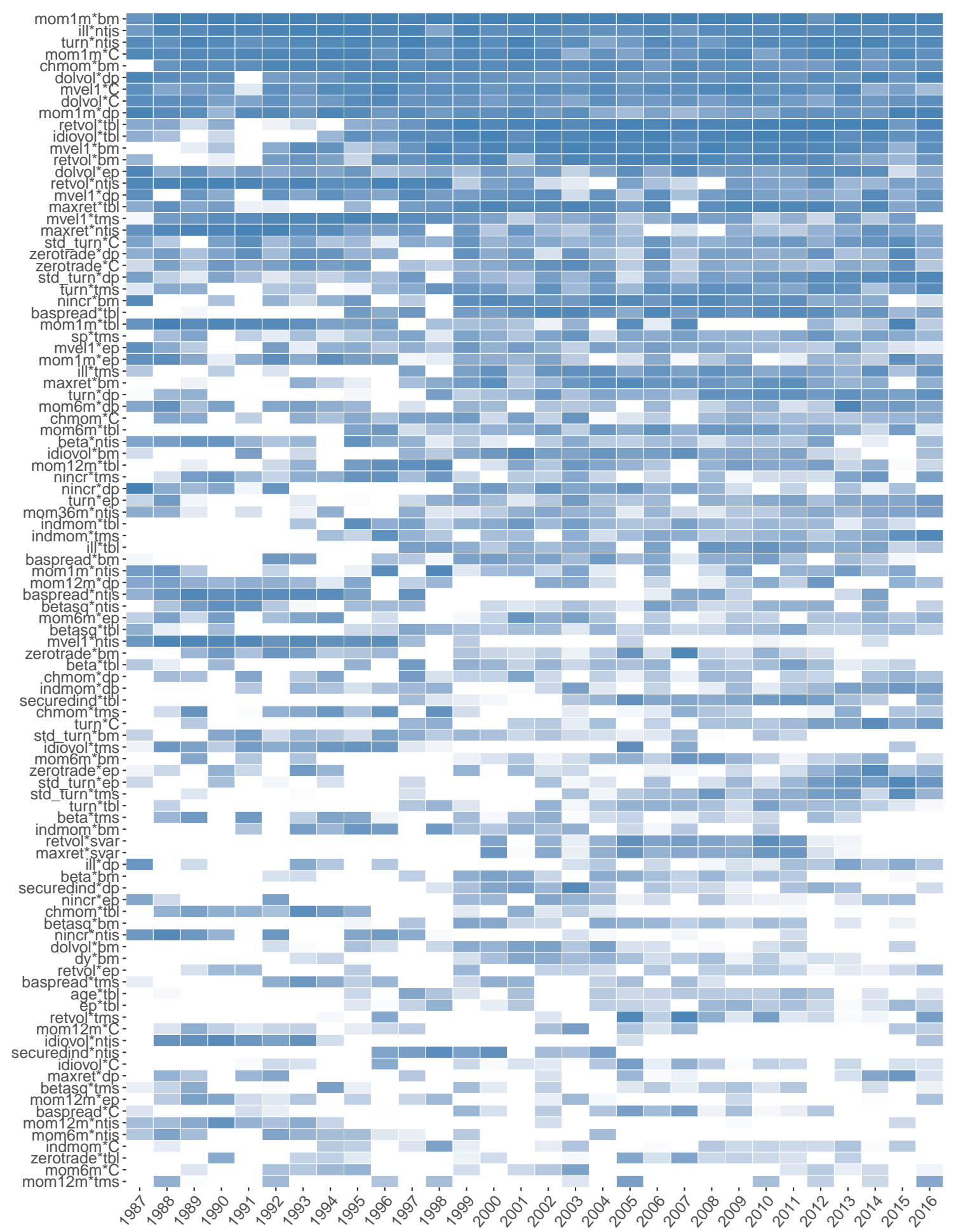

Note: Rankings of top 100 interactions between 94 stock-level stock characteristics and nine macro variables (including a constant, denoted $C$ ). The list of top 100 interactions is based on the analysis in Figure A.4. Color gradients indicate the most influential (dark blue) to least influential (white) interactions in the NN3 model in each training sample (the horizontal axis corresponds to the last year in each training sample). 
Figure A.6: Characteristic Importance at Annual Horizon

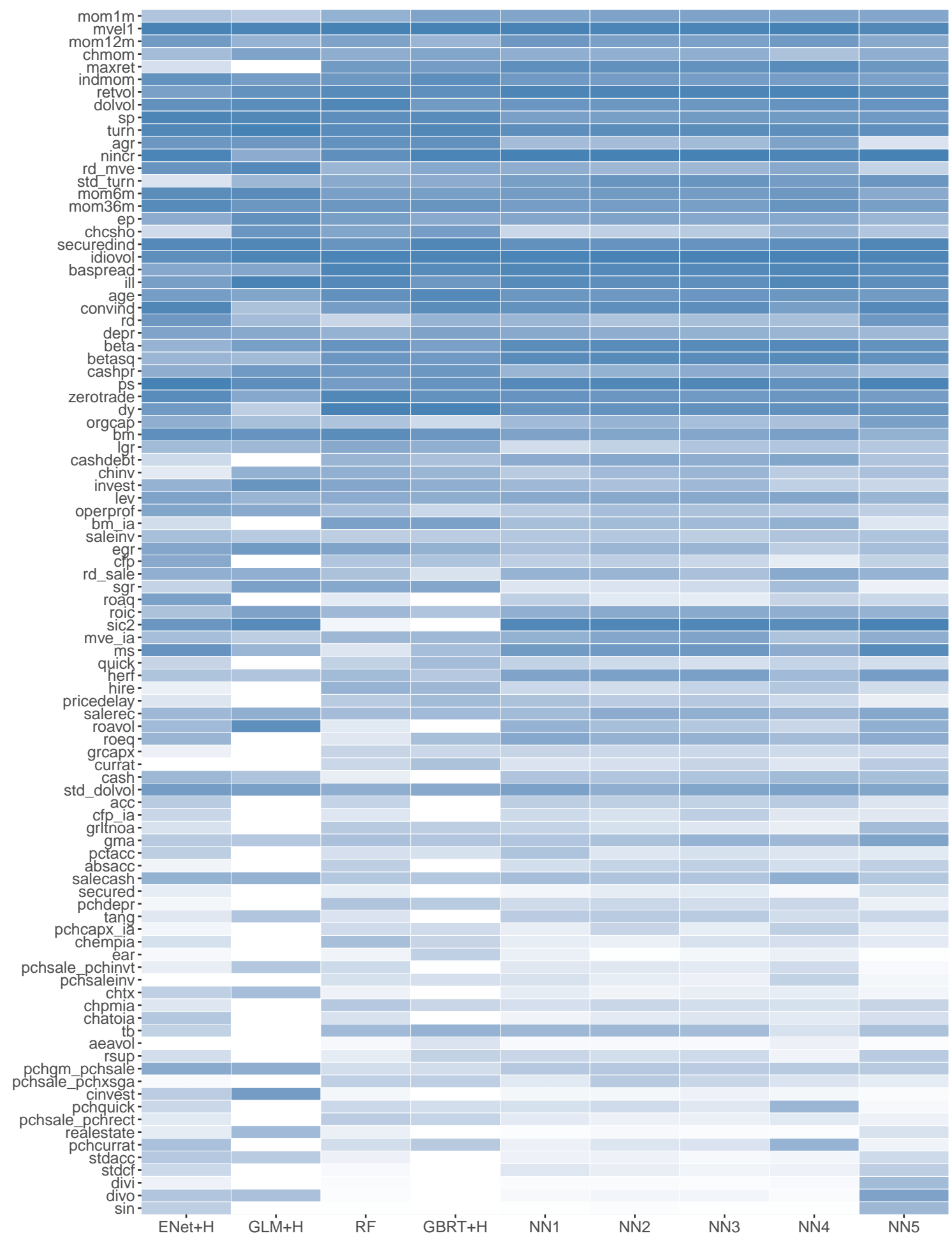

Note: This figure describes how each model ranks the 94 stock-level characteristics and the industry dummy (sic2) in terms of overall model contribution. Columns correspond to individual models, and color gradients within each column indicate the most influential (dark blue) to least influential (white) variables. Characteristics are sorted in the same order of Figure 5. The results are based on prediction at the annual horizon. 
Table A.8: Annual Portfolio-level Out-of-Sample Predictive $R^{2}$

\begin{tabular}{|c|c|c|c|c|c|c|c|c|c|c|c|c|}
\hline & $\begin{array}{c}\text { OLS-3 } \\
+\mathrm{H}\end{array}$ & PLS & PCR & $\begin{array}{c}\text { ENet } \\
+\mathrm{H}\end{array}$ & $\begin{array}{c}\text { GLM } \\
+\mathrm{H}\end{array}$ & $\mathrm{RF}$ & $\begin{array}{c}\text { GBRT } \\
+\mathrm{H}\end{array}$ & NN1 & NN2 & NN3 & NN4 & NN5 \\
\hline S\&P 500 & -4.90 & 0.43 & -7.17 & 0.26 & 2.07 & 8.80 & 7.28 & 9.99 & 12.02 & 15.68 & 15.30 & 13.15 \\
\hline Big Value & 1.83 & 4.88 & -4.04 & 3.62 & 0.49 & 9.50 & 5.86 & 8.76 & 8.54 & 12.42 & 9. & 7.56 \\
\hline Big Growth & -12.06 & -6.92 & -10.22 & -2.13 & 2.44 & 7.14 & 6.93 & 7.47 & 11.06 & 11.67 & 13.37 & 10.03 \\
\hline Big Neutral & -3.83 & 3.09 & -6.58 & 1.19 & 1.24 & 8.52 & 6.91 & 8.31 & 11.51 & 14.60 & 12.92 & 9.95 \\
\hline Small Value & 4.31 & 10.81 & 8.94 & 8.41 & 4.31 & 8.05 & 3.75 & 7.24 & 6.37 & 7.48 & 6.60 & 4.81 \\
\hline Small Growth & 2.49 & 2.87 & 3.19 & 0.21 & 0.03 & 6.20 & 2.13 & 3.96 & 5.52 & 6.84 & 2.60 & 7.23 \\
\hline Small Neutral & -1.52 & 5.21 & 2.10 & 2.29 & 2.29 & 4.18 & 1.78 & 6.46 & 5.55 & 6.68 & 3.69 & 6.14 \\
\hline Big Con & -10.42 & -2.42 & -9.77 & -3.77 & 5.17 & 8.44 & 5.26 & -1.31 & 8.64 & 9 . & 12.47 & 6.09 \\
\hline Big Agg & -1.65 & 1.89 & -4.72 & 1.36 & 2.00 & 7 & 6.67 & 11.00 & 11.74 & 13.08 & 11.27 & 10.67 \\
\hline Big Neutral & -9.18 & -1.62 & -9.42 & 2.03 & 2.43 & 22 & 8.39 & 10.88 & 13.03 & 15.61 & 15.75 & 13.56 \\
\hline tive & -0.38 & 6.36 & 5.01 & 3.19 & 2.35 & 4 & 0.62 & 5.31 & 5.39 & 5.97 & 4.22 & 4.71 \\
\hline Small $A$ & 3.33 & 5.12 & 2.88 & 1.04 & 0.37 & 6.43 & 3.23 & 2.50 & 4.50 & 5.50 & 1.47 & 6.56 \\
\hline Small Neutral & -0.53 & 5.84 & 3.52 & 4.46 & 3.59 & 7.08 & 2.96 & 8.41 & 7.13 & 8.68 & 5.77 & 8.47 \\
\hline Big I & -7.53 & -2.55 & -9.18 & 1.33 & 5.42 & 7.61 & 6.60 & 12.55 & 12.04 & 13.92 & 15.29 & 13.35 \\
\hline Big Weak & -3.40 & 3.09 & -7.15 & -1.02 & -1.12 & 9.62 & 7.62 & 4.41 & 9.95 & 11.39 & 11.73 & 8.40 \\
\hline Big Neutral & -4.17 & 5.46 & -4.57 & -3.18 & -2.12 & 6.24 & 4.47 & 4.18 & 6.23 & 9.47 & 3.70 & 2.95 \\
\hline Small Robust & -2.37 & 0.93 & -0.20 & 0.76 & 3.72 & 0.41 & -0.87 & 2.92 & 3.67 & 4.47 & 0.86 & 4.19 \\
\hline Small Weak & 3.88 & 9.89 & 5.68 & 2.15 & -1.11 & 7.53 & 3.10 & -0.48 & 1.53 & 2.96 & 1.61 & 1.08 \\
\hline Small Neutral & 3.00 & 7.99 & 4.40 & 4.60 & 3.58 & 9.21 & 5.75 & 10.03 & 7.39 & 9.82 & 7.06 & 9.09 \\
\hline Big Up & -23.55 & -11.77 & -19.16 & -5.11 & 0.52 & 6.15 & 6.21 & 4.26 & 11.44 & 11.11 & 14.48 & 10.62 \\
\hline Big Down & -4.66 & 0.39 & -2.79 & -0.15 & 0.71 & 7.64 & 5.53 & 3.58 & 8.78 & 9.54 & 10.32 & 6.79 \\
\hline Big Medium & 6.26 & 10.24 & 7.36 & 6.25 & 3.83 & 7.73 & 5.38 & 8.74 & 9.61 & 11.36 & 9.96 & 6.22 \\
\hline Small Up & -6.68 & 3.82 & 0.71 & -2.83 & 1.57 & 1.84 & -0.19 & -4.22 & 0.70 & 1.12 & -1.42 & 2.83 \\
\hline Small Down & 2.80 & 5.59 & 4.84 & 2.87 & 0.50 & 7.23 & 3.49 & 3.24 & 4.63 & 5.90 & 3.28 & 5.22 \\
\hline Small Medium & -2.92 & -0.49 & -1.70 & -1.80 & 0.81 & 2.00 & -0.40 & -1.64 & 1.96 & 1.79 & 0.51 & 3.49 \\
\hline SMB & 3.77 & 4.23 & 8.26 & 4.22 & 6.96 & 6.54 & 4.27 & 0.05 & 1.31 & 2.59 & 4.33 & 4.45 \\
\hline HML & 3.01 & -0.52 & 4.08 & -0.15 & 6.33 & 7.02 & 2.17 & 9.14 & 8.09 & 7.86 & 3.97 & 3.63 \\
\hline RMW & 4.66 & 17.11 & 6.67 & 1.19 & 3.45 & 3.51 & 5.31 & 7.03 & 5.03 & 3.58 & 0.61 & 0.90 \\
\hline CMA & 4.50 & -1.52 & 7.94 & -9.01 & 7.69 & 1.73 & -8.36 & 5.89 & 7.27 & 0.93 & -7.18 & -8.11 \\
\hline MOM & -27.52 & -12.44 & -5.62 & -16.27 & -8.06 & -7.57 & -8.29 & -12.78 & -8.71 & -7.35 & -6.45 & -6.74 \\
\hline
\end{tabular}

Note: In this table, we report the out-of-sample predictive $R^{2}$ s for 25 portfolios using OLS with size, book-to-market, and momentum, OLS-3, PLS, PCR, elastic net (ENet), generalized linear model with group lasso (GLM), random forest $(\mathrm{RF})$, gradient boosted regression trees (GBRT), and five architectures of neural networks (NN1,.., NN5), respectively. " $+\mathrm{H}$ " indicates the use of Huber loss instead of the $l_{2}$ loss. The 25 portfolios are $3 \times 2$ size double-sorted portfolios used in the construction of the Fama-French value, investment, and profitability factors, as well as momentum. The results are based on prediction at the annual horizon. 
Table A.9: Performance of Machine Learning Portfolios (Equally Weighted)

\begin{tabular}{|c|c|c|c|c|c|c|c|c|c|c|c|c|}
\hline & \multicolumn{4}{|c|}{$\mathrm{OLS}-3+\mathrm{H}$} & \multicolumn{4}{|c|}{ PLS } & \multicolumn{4}{|c|}{ PCR } \\
\hline & Pred & Avg & Std & SR & Pred & Avg & Std & SR & Pred & Avg & Std & $\mathrm{SR}$ \\
\hline Low(L) & -0.14 & 0.11 & 7.99 & 0.05 & -0.83 & -0.26 & 6.41 & -0.14 & -0.71 & -0.65 & 7.04 & -0.32 \\
\hline 2 & 0.17 & 0.35 & 6.81 & 0.18 & -0.20 & 0.19 & 5.92 & 0.11 & -0.11 & 0.16 & 6.23 & 0.09 \\
\hline 3 & 0.35 & 0.44 & 6.09 & 0.25 & 0.12 & 0.40 & 5.49 & 0.25 & 0.19 & 0.40 & 5.67 & 0.25 \\
\hline 4 & 0.49 & 0.63 & 5.61 & 0.39 & 0.39 & 0.67 & 5.06 & 0.46 & 0.42 & 0.58 & 5.45 & 0.37 \\
\hline 5 & 0.63 & 0.73 & 5.24 & 0.49 & 0.62 & 0.69 & 5.14 & 0.47 & 0.63 & 0.72 & 5.11 & 0.49 \\
\hline 6 & 0.75 & 0.83 & 4.88 & 0.59 & 0.84 & 0.77 & 5.14 & 0.52 & 0.81 & 0.80 & 4.98 & 0.55 \\
\hline 7 & 0.88 & 0.75 & 4.73 & 0.55 & 1.06 & 0.88 & 5.12 & 0.60 & 1.01 & 0.98 & 5.02 & 0.68 \\
\hline 8 & 1.03 & 0.80 & 4.72 & 0.59 & 1.32 & 1.01 & 5.29 & 0.66 & 1.23 & 1.08 & 5.02 & 0.75 \\
\hline 9 & 1.22 & 1.14 & 4.73 & 0.83 & 1.67 & 1.28 & 5.60 & 0.79 & 1.52 & 1.33 & 5.28 & 0.88 \\
\hline $\operatorname{High}(\mathrm{H})$ & 1.60 & 1.45 & 5.21 & 0.96 & 2.38 & 1.82 & 6.16 & 1.02 & 2.12 & 1.81 & 5.93 & 1.06 \\
\hline \multirow[t]{3}{*}{ H-L } & 1.73 & 1.34 & 5.59 & 0.83 & 3.21 & 2.08 & 4.89 & 1.47 & 2.83 & 2.45 & 4.51 & 1.89 \\
\hline & \multicolumn{4}{|c|}{ ENet $+\mathrm{H}$} & \multicolumn{4}{|c|}{$\mathrm{GLM}+\mathrm{H}$} & \multicolumn{4}{|c|}{$\mathrm{RF}$} \\
\hline & Pred & Avg & Std & $\mathrm{SR}$ & Pred & Avg & Std & $\mathrm{SR}$ & Pred & Avg & Std & $\mathrm{SR}$ \\
\hline Low $(\mathrm{L})$ & -0.04 & -0.24 & 6.43 & -0.13 & -0.49 & -0.50 & 6.81 & -0.25 & 0.26 & -0.48 & 7.16 & -0.23 \\
\hline 2 & 0.27 & 0.44 & 5.90 & 0.26 & 0.01 & 0.32 & 5.80 & 0.19 & 0.44 & 0.24 & 5.67 & 0.15 \\
\hline 3 & 0.44 & 0.52 & 5.27 & 0.34 & 0.29 & 0.56 & 5.46 & 0.36 & 0.53 & 0.55 & 5.36 & 0.36 \\
\hline 4 & 0.59 & 0.70 & 4.73 & 0.51 & 0.50 & 0.61 & 5.22 & 0.41 & 0.60 & 0.62 & 5.15 & 0.42 \\
\hline 5 & 0.73 & 0.71 & 4.94 & 0.49 & 0.68 & 0.72 & 5.11 & 0.49 & 0.67 & 0.66 & 5.11 & 0.44 \\
\hline 6 & 0.87 & 0.79 & 5.00 & 0.55 & 0.84 & 0.78 & 5.12 & 0.53 & 0.73 & 0.77 & 5.13 & 0.52 \\
\hline 7 & 1.01 & 0.85 & 5.21 & 0.56 & 1.00 & 0.78 & 5.06 & 0.54 & 0.80 & 0.74 & 5.10 & 0.50 \\
\hline 8 & 1.17 & 0.88 & 5.47 & 0.56 & 1.18 & 0.89 & 5.14 & 0.60 & 0.87 & 0.99 & 5.29 & 0.65 \\
\hline 9 & 1.36 & 0.85 & 5.90 & 0.50 & 1.41 & 1.25 & 5.80 & 0.75 & 0.97 & 1.22 & 5.67 & 0.74 \\
\hline $\operatorname{High}(\mathrm{H})$ & 1.72 & 1.86 & 7.27 & 0.89 & 1.89 & 1.81 & 6.57 & 0.96 & 1.20 & 1.90 & 7.03 & 0.94 \\
\hline \multirow[t]{3}{*}{$\mathrm{H}-\mathrm{L}$} & 1.76 & 2.11 & 5.50 & 1.33 & 2.38 & 2.31 & 4.41 & 1.82 & 0.94 & 2.38 & 5.57 & 1.48 \\
\hline & \multicolumn{4}{|c|}{$\mathrm{GBRT}+\mathrm{H}$} & \multicolumn{4}{|c|}{ NN1 } & \multicolumn{4}{|c|}{$\mathrm{NN} 2$} \\
\hline & Pred & Avg & Std & $\mathrm{SR}$ & Pred & Avg & Std & $\mathrm{SR}$ & Pred & Avg & Std & $\mathrm{SR}$ \\
\hline Low $(\mathrm{L})$ & -0.49 & -0.37 & 6.46 & -0.20 & -0.45 & -0.78 & 7.43 & -0.36 & -0.32 & -1.01 & 7.79 & -0.45 \\
\hline 2 & -0.16 & 0.42 & 5.80 & 0.25 & 0.15 & 0.22 & 6.24 & 0.12 & 0.20 & 0.17 & 6.34 & 0.09 \\
\hline 3 & 0.02 & 0.56 & 5.31 & 0.36 & 0.43 & 0.47 & 5.55 & 0.29 & 0.43 & 0.52 & 5.49 & 0.33 \\
\hline 4 & 0.17 & 0.74 & 5.43 & 0.47 & 0.64 & 0.64 & 5.00 & 0.45 & 0.59 & 0.71 & 5.02 & 0.49 \\
\hline 5 & 0.33 & 0.63 & 5.31 & 0.41 & 0.80 & 0.80 & 4.76 & 0.58 & 0.72 & 0.76 & 4.60 & 0.57 \\
\hline 6 & 0.46 & 0.83 & 5.23 & 0.55 & 0.95 & 0.85 & 4.63 & 0.63 & 0.84 & 0.81 & 4.52 & 0.62 \\
\hline 7 & 0.59 & 0.67 & 5.13 & 0.45 & 1.12 & 0.84 & 4.66 & 0.62 & 0.97 & 0.94 & 4.61 & 0.70 \\
\hline 8 & 0.72 & 0.82 & 5.08 & 0.56 & 1.32 & 0.88 & 4.95 & 0.62 & 1.14 & 0.92 & 4.86 & 0.66 \\
\hline 9 & 0.88 & 1.12 & 5.41 & 0.72 & 1.63 & 1.17 & 5.62 & 0.72 & 1.41 & 1.10 & 5.55 & 0.69 \\
\hline $\operatorname{High}(\mathrm{H})$ & 1.19 & 1.77 & 6.69 & 0.92 & 2.43 & 2.13 & 7.34 & 1.00 & 2.25 & 2.30 & 7.81 & 1.02 \\
\hline \multirow[t]{3}{*}{$\mathrm{H}-\mathrm{L}$} & 1.68 & 2.14 & 4.28 & 1.73 & 2.89 & 2.91 & 4.72 & 2.13 & 2.57 & 3.31 & 4.92 & 2.33 \\
\hline & \multicolumn{4}{|c|}{ NN3 } & \multicolumn{4}{|c|}{ NN4 } & \multicolumn{4}{|c|}{ NN5 } \\
\hline & Pred & Avg & Std & $\mathrm{SR}$ & Pred & Avg & Std & SR & Pred & Avg & Std & $\mathrm{SR}$ \\
\hline Low $(\mathrm{L})$ & -0.31 & -0.92 & 7.94 & -0.40 & -0.19 & -0.95 & 7.83 & -0.42 & -0.08 & -0.83 & 7.92 & -0.36 \\
\hline 2 & 0.22 & 0.16 & 6.46 & 0.09 & 0.29 & 0.17 & 6.50 & 0.09 & 0.33 & 0.24 & 6.64 & 0.12 \\
\hline 3 & 0.45 & 0.44 & 5.40 & 0.28 & 0.49 & 0.45 & 5.58 & 0.28 & 0.51 & 0.53 & 5.65 & 0.32 \\
\hline 4 & 0.60 & 0.66 & 4.83 & 0.48 & 0.62 & 0.57 & 4.94 & 0.40 & 0.62 & 0.59 & 4.91 & 0.41 \\
\hline 5 & 0.73 & 0.77 & 4.58 & 0.58 & 0.72 & 0.70 & 4.57 & 0.53 & 0.71 & 0.68 & 4.56 & 0.51 \\
\hline 6 & 0.85 & 0.81 & 4.47 & 0.63 & 0.81 & 0.75 & 4.42 & 0.59 & 0.80 & 0.76 & 4.43 & 0.60 \\
\hline 7 & 0.97 & 0.86 & 4.62 & 0.64 & 0.91 & 0.86 & 4.47 & 0.67 & 0.88 & 0.88 & 4.60 & 0.66 \\
\hline 8 & 1.12 & 0.93 & 4.82 & 0.67 & 1.04 & 1.06 & 4.82 & 0.76 & 1.01 & 0.95 & 4.90 & 0.67 \\
\hline 9 & 1.38 & 1.18 & 5.51 & 0.74 & 1.28 & 1.24 & 5.57 & 0.77 & 1.25 & 1.17 & 5.60 & 0.73 \\
\hline $\operatorname{High}(\mathrm{H})$ & 2.28 & 2.35 & 8.11 & 1.00 & 2.16 & 2.37 & 8.03 & 1.02 & 2.08 & 2.27 & 7.95 & 0.99 \\
\hline $\mathrm{H}-\mathrm{L}$ & 2.58 & 3.27 & 4.80 & 2.36 & 2.35 & 3.33 & 4.71 & 2.45 & 2.16 & 3.09 & 4.98 & 2.15 \\
\hline
\end{tabular}

Note: Performance of equal-weight decile portfolios sorted on out-of-sample machine learning return forecasts. "Pred", "Avg", "Std", and "SR" report the predicted monthly returns for each decile, the average realized monthly returns, their realized standard deviations, and annualized Sharpe ratios, respectively. 
Table A.10: Performance of Machine Learning Portfolios (Equally Weighted, Excluding Microcaps)

\begin{tabular}{|c|c|c|c|c|c|c|c|c|c|c|c|c|}
\hline & \multicolumn{4}{|c|}{ OLS-3+H } & \multicolumn{4}{|c|}{ PLS } & \multicolumn{4}{|c|}{ PCR } \\
\hline & Pred & Avg & Std & $\mathrm{SR}$ & Pred & Avg & Std & SR & Pred & Avg & Std & SR \\
\hline Low(L) & -0.17 & 0.00 & 7.97 & 0.00 & -0.88 & -0.33 & 6.59 & -0.17 & -0.72 & -0.50 & 7.04 & -0.25 \\
\hline 2 & 0.12 & 0.19 & 6.53 & 0.10 & -0.26 & 0.27 & 5.83 & 0.16 & -0.13 & 0.16 & 6.14 & 0.09 \\
\hline 3 & 0.31 & 0.40 & 5.72 & 0.24 & 0.06 & 0.35 & 5.41 & 0.22 & 0.16 & 0.36 & 5.52 & 0.22 \\
\hline 4 & 0.45 & 0.52 & 5.32 & 0.34 & 0.31 & 0.54 & 5.16 & 0.36 & 0.39 & 0.52 & 5.21 & 0.35 \\
\hline 5 & 0.58 & 0.63 & 4.96 & 0.44 & 0.54 & 0.66 & 5.01 & 0.46 & 0.59 & 0.63 & 4.94 & 0.44 \\
\hline 6 & 0.70 & 0.63 & 4.71 & 0.46 & 0.75 & 0.70 & 4.97 & 0.49 & 0.77 & 0.71 & 4.83 & 0.51 \\
\hline 7 & 0.82 & 0.66 & 4.64 & 0.49 & 0.96 & 0.82 & 4.71 & 0.60 & 0.96 & 0.76 & 4.80 & 0.55 \\
\hline 8 & 0.96 & 0.75 & 4.70 & 0.56 & 1.21 & 0.85 & 5.12 & 0.57 & 1.17 & 0.95 & 4.84 & 0.68 \\
\hline 9 & 1.15 & 1.04 & 4.95 & 0.73 & 1.53 & 1.02 & 5.32 & 0.66 & 1.46 & 1.09 & 5.14 & 0.74 \\
\hline $\operatorname{High}(\mathrm{H})$ & 1.47 & 1.33 & 5.35 & 0.86 & 2.21 & 1.33 & 5.87 & 0.78 & 2.03 & 1.47 & 5.83 & 0.87 \\
\hline \multirow[t]{3}{*}{$\underline{\mathrm{H}-\mathrm{L}}$} & 1.64 & 1.32 & 5.66 & 0.81 & 3.09 & 1.66 & 4.69 & 1.22 & 2.75 & 1.97 & 4.61 & 1.48 \\
\hline & \multicolumn{4}{|c|}{ ENet $+\mathrm{H}$} & \multicolumn{4}{|c|}{$\mathrm{GLM}+\mathrm{H}$} & \multicolumn{4}{|c|}{$\mathrm{RF}$} \\
\hline & Pred & Avg & Std & SR & Pred & Avg & Std & SR & Pred & Avg & Std & $\mathrm{SR}$ \\
\hline Low $(\mathrm{L})$ & -0.05 & -0.23 & 6.51 & -0.12 & -0.51 & -0.35 & 6.81 & -0.18 & 0.27 & -0.43 & 7.03 & -0.21 \\
\hline 2 & 0.25 & 0.42 & 5.72 & 0.26 & -0.03 & 0.32 & 5.71 & 0.20 & 0.44 & 0.23 & 5.58 & 0.15 \\
\hline 3 & 0.42 & 0.53 & 5.14 & 0.36 & 0.25 & 0.54 & 5.34 & 0.35 & 0.52 & 0.50 & 5.19 & 0.33 \\
\hline 4 & 0.56 & 0.60 & 4.82 & 0.43 & 0.45 & 0.59 & 5.12 & 0.40 & 0.59 & 0.58 & 5.04 & 0.40 \\
\hline 5 & 0.69 & 0.69 & 4.80 & 0.50 & 0.63 & 0.65 & 4.98 & 0.45 & 0.66 & 0.58 & 4.97 & 0.41 \\
\hline 6 & 0.82 & 0.73 & 4.89 & 0.52 & 0.79 & 0.68 & 4.96 & 0.48 & 0.72 & 0.65 & 5.04 & 0.45 \\
\hline 7 & 0.96 & 0.83 & 4.74 & 0.61 & 0.95 & 0.70 & 4.91 & 0.49 & 0.78 & 0.65 & 4.99 & 0.45 \\
\hline 8 & 1.11 & 0.77 & 5.31 & 0.50 & 1.12 & 0.75 & 4.95 & 0.53 & 0.85 & 0.85 & 5.02 & 0.58 \\
\hline 9 & 1.30 & 0.78 & 5.74 & 0.47 & 1.34 & 0.95 & 5.30 & 0.62 & 0.92 & 1.08 & 5.34 & 0.70 \\
\hline $\operatorname{High}(\mathrm{H})$ & 1.65 & 1.04 & 6.78 & 0.53 & 1.79 & 1.31 & 6.33 & 0.72 & 1.09 & 1.43 & 6.65 & 0.74 \\
\hline \multirow[t]{3}{*}{$\underline{\mathrm{H}-\mathrm{L}}$} & 1.70 & 1.27 & 4.90 & 0.90 & 2.30 & 1.65 & 4.44 & 1.29 & 0.81 & 1.86 & 5.25 & 1.22 \\
\hline & \multicolumn{4}{|c|}{$\mathrm{GBRT}+\mathrm{H}$} & \multicolumn{4}{|c|}{ NN1 } & \multicolumn{4}{|c|}{$\mathrm{NN} 2$} \\
\hline & Pred & Avg & Std & $\mathrm{SR}$ & Pred & Avg & Std & SR & Pred & Avg & Std & SR \\
\hline Low $(\mathrm{L})$ & -0.47 & -0.28 & 6.25 & -0.15 & -0.47 & -0.76 & 7.48 & -0.35 & -0.33 & -0.92 & 8.00 & -0.40 \\
\hline 2 & -0.15 & 0.38 & 5.55 & 0.24 & 0.12 & 0.20 & 6.36 & 0.11 & 0.19 & 0.20 & 6.51 & 0.10 \\
\hline 3 & 0.02 & 0.52 & 5.22 & 0.34 & 0.40 & 0.48 & 5.54 & 0.30 & 0.41 & 0.55 & 5.63 & 0.34 \\
\hline 4 & 0.17 & 0.67 & 5.31 & 0.44 & 0.59 & 0.63 & 5.01 & 0.43 & 0.56 & 0.70 & 5.03 & 0.48 \\
\hline 5 & 0.32 & 0.55 & 5.24 & 0.36 & 0.74 & 0.72 & 4.76 & 0.53 & 0.68 & 0.74 & 4.59 & 0.56 \\
\hline 6 & 0.45 & 0.76 & 4.95 & 0.54 & 0.87 & 0.85 & 4.61 & 0.64 & 0.79 & 0.84 & 4.49 & 0.65 \\
\hline 7 & 0.57 & 0.52 & 5.10 & 0.35 & 1.01 & 0.87 & 4.60 & 0.65 & 0.89 & 0.90 & 4.51 & 0.69 \\
\hline 8 & 0.69 & 0.70 & 4.90 & 0.50 & 1.16 & 0.85 & 4.68 & 0.63 & 1.02 & 0.93 & 4.69 & 0.68 \\
\hline 9 & 0.84 & 1.02 & 5.26 & 0.67 & 1.38 & 1.00 & 5.13 & 0.68 & 1.19 & 0.96 & 4.99 & 0.67 \\
\hline $\operatorname{High}(\mathrm{H})$ & 1.10 & 1.30 & 6.25 & 0.72 & 1.91 & 1.29 & 6.25 & 0.72 & 1.68 & 1.26 & 6.22 & 0.70 \\
\hline \multirow[t]{3}{*}{$\underline{\mathrm{H}-\mathrm{L}}$} & 1.57 & 1.58 & 3.86 & 1.42 & 2.38 & 2.05 & 4.50 & 1.58 & 2.01 & 2.18 & 4.74 & 1.60 \\
\hline & \multicolumn{4}{|c|}{ NN3 } & \multicolumn{4}{|c|}{ NN4 } & \multicolumn{4}{|c|}{ NN5 } \\
\hline & Pred & Avg & Std & SR & Pred & Avg & Std & SR & Pred & Avg & Std & SR \\
\hline Low $(\mathrm{L})$ & -0.31 & -0.82 & 8.18 & -0.35 & -0.19 & -0.87 & 8.05 & -0.38 & -0.08 & -0.75 & 8.11 & -0.32 \\
\hline 2 & 0.20 & 0.16 & 6.55 & 0.08 & 0.28 & 0.23 & 6.68 & 0.12 & 0.32 & 0.22 & 6.75 & 0.12 \\
\hline 3 & 0.43 & 0.46 & 5.51 & 0.29 & 0.47 & 0.45 & 5.61 & 0.28 & 0.49 & 0.51 & 5.70 & 0.31 \\
\hline 4 & 0.57 & 0.66 & 4.86 & 0.47 & 0.59 & 0.65 & 4.93 & 0.45 & 0.61 & 0.58 & 4.98 & 0.40 \\
\hline 5 & 0.69 & 0.76 & 4.63 & 0.57 & 0.68 & 0.65 & 4.60 & 0.49 & 0.69 & 0.69 & 4.55 & 0.52 \\
\hline 6 & 0.79 & 0.79 & 4.44 & 0.61 & 0.76 & 0.71 & 4.48 & 0.55 & 0.76 & 0.76 & 4.43 & 0.60 \\
\hline 7 & 0.89 & 0.87 & 4.48 & 0.67 & 0.84 & 0.90 & 4.45 & 0.70 & 0.83 & 0.84 & 4.45 & 0.65 \\
\hline 8 & 1.01 & 0.91 & 4.71 & 0.67 & 0.94 & 0.92 & 4.59 & 0.70 & 0.91 & 0.92 & 4.70 & 0.68 \\
\hline 9 & 1.17 & 1.00 & 5.02 & 0.69 & 1.07 & 1.13 & 5.00 & 0.78 & 1.04 & 1.02 & 5.10 & 0.69 \\
\hline $\operatorname{High}(\mathrm{H})$ & 1.64 & 1.37 & 6.34 & 0.75 & 1.52 & 1.39 & 6.37 & 0.75 & 1.48 & 1.36 & 6.34 & 0.74 \\
\hline $\mathrm{H}-\mathrm{L}$ & 1.95 & 2.19 & 4.84 & 1.57 & 1.70 & 2.26 & 4.63 & 1.69 & 1.56 & 2.11 & 4.95 & 1.48 \\
\hline
\end{tabular}

Note: In this table, we report the performance of prediction-sorted portfolios over the 30-year out-of-sample testing period. All but tiny stocks (excluding stocks below 20th percentile on NYSE cap weights) are sorted into deciles based on their predicted returns for the next month. Column "Pred", "Avg", "Std", and "SR" provide the predicted monthly returns for each decile, the average realized monthly returns, their standard deviations, and Sharpe ratios, respectively. All portfolios are value weighted. 
Table A.11: OLS Benchmark Models

\begin{tabular}{|c|c|c|c|c|c|}
\hline \multirow[b]{2}{*}{ Model } & \multicolumn{2}{|c|}{$R^{2}$} & \multicolumn{2}{|c|}{ Sharpe Ratio } & \multirow[b]{2}{*}{ Description } \\
\hline & Stock & S\&P 500 & Equal-weight & Value-weight & \\
\hline OLS-3 & 0.16 & -0.22 & 0.83 & 0.61 & mom12m, size, bm \\
\hline OLS-7 & 0.18 & 0.24 & 1.12 & 0.74 & OLS-3 plus acc, roaq, agr, egr \\
\hline OLS-15 & 0.19 & 0.68 & 1.15 & 0.86 & OLS-7 plus dy, mom $36 \mathrm{~m}$, beta, retvol, turn, lev, sp \\
\hline $\mathrm{RF}$ & 0.33 & 1.37 & 1.48 & 0.98 & \\
\hline NN3 & 0.40 & 1.80 & 2.36 & 1.20 & \\
\hline
\end{tabular}

Note: In this table, we report the out-of-sample performance of three different OLS benchmark models recommended by Lewellen (2015) with either three, seven, or 15 predictors. We report predictive $R^{2}$ for the stock-level panel and the S\&P 500 index. We report long-short decile spread Sharpe ratios with equal-weight and value-weight formation. For comparison, we also report the performance the NN3 and random forest models.

Figure A.7: Cumulative Return of Machine Learning Portfolios (Equally Weighted)

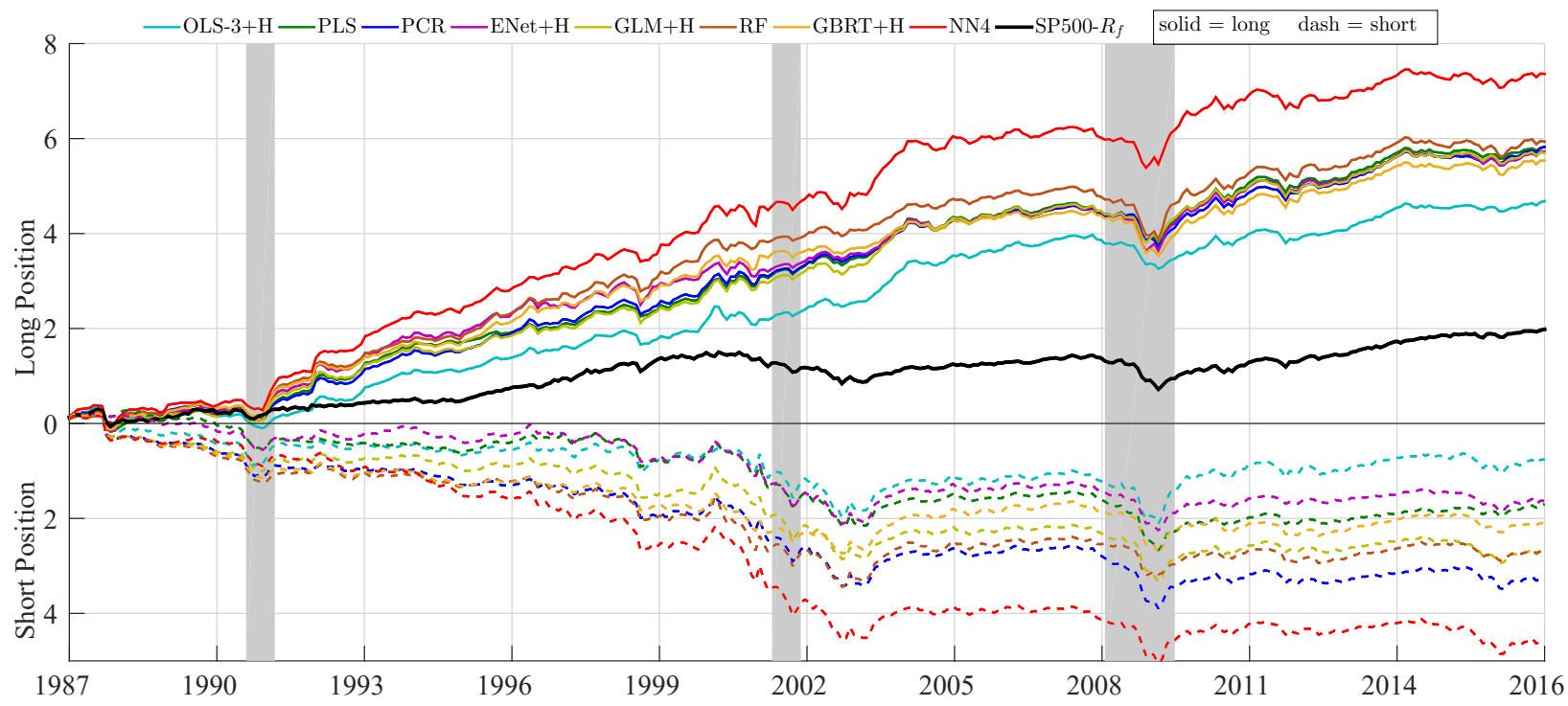

Note: Cumulative log returns of portfolios sorted on out-of-sample machine learning return forecasts. The solid and dash lines represent long (top decile) and short (bottom decile) positions, respectively. The shaded periods show NBER recession dates. All portfolios are equally weighted. 